\title{
LECTURE NOTES Special topics in plasma confinement
}

\author{
J. B. Taylor ${ }^{1,2}$ and S. L. Newton ${ }^{2, \dagger}$ \\ ${ }^{1}$ Rudolf Peierls Centre for Theoretical Physics, University of Oxford, Oxford OX1 3NP, UK \\ ${ }^{2}$ CCFE, Culham Science Centre, Abingdon, Oxon OX14 3DB, UK
}

(Received 3 February 2015; revised 22 April 2015; accepted 27 May 2015)

These notes are based on lectures given by one of us (J.B.T.) at the University of Texas in Austin in 1991. Part I concerns some basic features of plasma confinement by magnetic fields as an introduction to an account of plasma relaxation in Part II. Part III discusses confinement by magnetic mirrors, especially minimum- $B$ systems. It also includes a general discussion of adiabatic invariants and of the principle of maximal ordering in perturbation theory. Part IV is devoted to the analysis of perturbations in toroidal plasmas and the stability of ballooning modes.

\section{PREFACE}

The theory of plasma confinement is complicated and incomplete. Because current textbooks do not include material at the frontier of research, it is hard for students and researchers to become familiar with the state of the art. Bryan Taylor's notes are presented here to start filling that gap. Although based on lectures from over twenty years ago, they provide much needed clarity in selected areas of current plasma confinement research. Of course much of the original work on which these notes are based is by Bryan Taylor and collaborators; but here, he and Sarah Newton draw the many threads together. Indeed, while some may find the results familiar, the insight is fresh and enlightening. The research frontier is just beyond these notes. Some of the outstanding questions are posed and some are left to the reader to discern. For example, we still need to know: how fast plasmas relax; how three-dimensional reconnection works; how ballooning modes saturate and; how minimum B ideas can improve toroidal devices. The insights here will help anyone wanting to answer those and other questions. 


\section{Contents}

Preface 1

$\begin{array}{ll}\text { Foreword } & 4\end{array}$

$\begin{array}{ll}\text { Part I } & 4\end{array}$

1 Introduction $\quad 4$

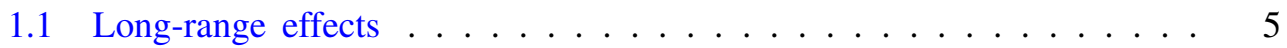

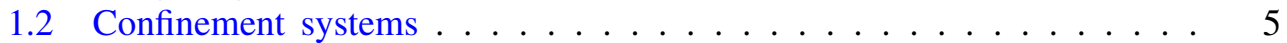

1.3 Plasma models ..................... 5

1.3.1 Fluid model ........................ 5

1.3.2 Guiding centre model ........................ 6

1.3.3 Kinetic theory models ............... 6

2 Toroidal confinement $\quad \mathbf{8}$

2.1 Magnetic surfaces . . . . . . . . . . . . . . . . . 9

2.2 Magnetic surfaces and the Grad-Shafranov equation . . . . . . . . . 12

2.2.1 Cylindrical . . . . . . . . . . . . . . . . 12

2.2 .2 Toroidal . . . . . . . . . . . . . . . . 13

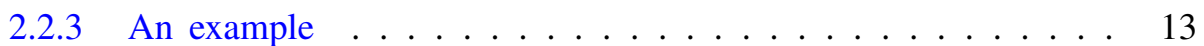

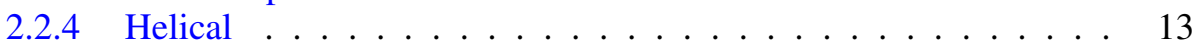

2.3 Rotational transform .................... 15

3 Perturbations of symmetry 15

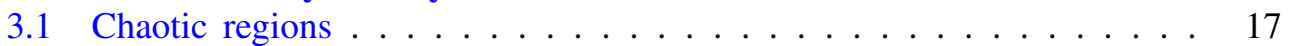

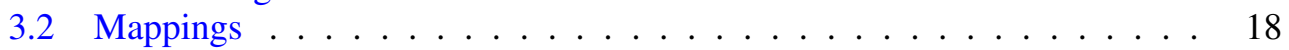

4 Motion of lines of force $\quad 19$

4.1 Fluid model with resistivity .................. . . . 20

4.2 Effect of field line preservation . . . . . . . . . . . . . 22

5 Resistive plasmas $\quad 23$

5.1 Resistive instabilities: the tearing mode .............. 23

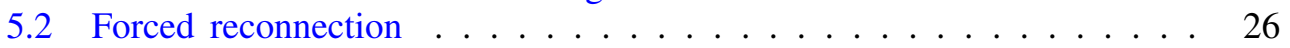

$\begin{array}{llr}6 & \text { Conclusion } & 27\end{array}$

$\begin{array}{lr}\text { Part II } & 28\end{array}$

7 Plasma relaxation $\quad \mathbf{2 8}$

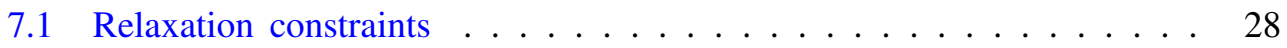

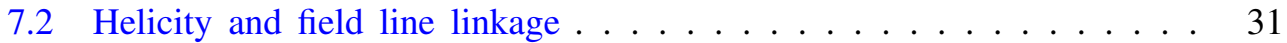

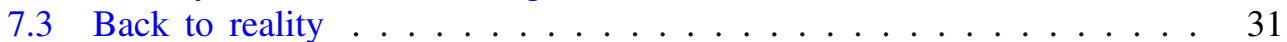

7.4 Uniqueness of relaxed states . . . . . . . . . . . . . . . 32

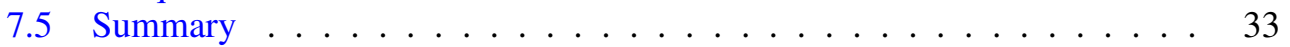

8 Relaxed states in toroidal pinch $\quad 33$

8.1 Spontaneous field reversal . . . . . . . . . . . . . 33 
9 Energetics of toroidal relaxed states $\quad 34$

9.1 Primitive and mixed states . . . . . . . . . . . . . . . 37

9.2 Current saturation and spontaneous symmetry breaking ....... 38

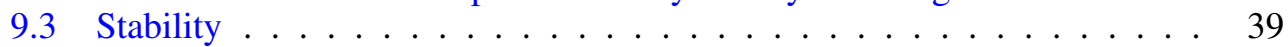

10 The multipinch $\quad 40$

10.1 Nature of the eigenfunctions in multipinch . . . . . . . . . . . . 42

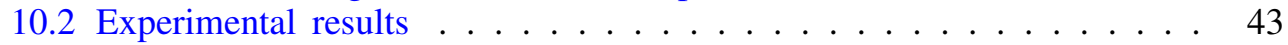

10.3 Flux generation ..................... . . . . 44

11 Spherical systems I: spheromak 44

11.1 Relaxed states of a spheromak . . . . . . . . . . . . . . . . 45

11.2 Spheromak eigenfunctions ................. . . 46

11.3 Experimental data . . . . . . . . . . . . . . . 47

12 Spherical systems II: flux core spheromak $\quad 47$

12.1 Relative helicity . . . . . . . . . . . . . . . . . 48

12.2 Relaxed states of flux core spheromak . . . . . . . . . . . 5 51

12.3 Experimental control of relaxed states . . . . . . . . . . . . 52

12.4 The kinked Z-pinch . . . . . . . . . . . . . . 53

13 Helicity injection $\quad 54$

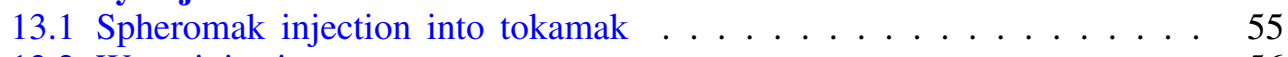

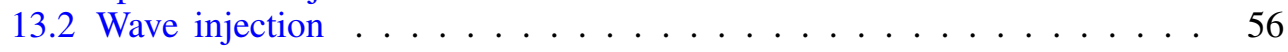

14 General theory of relaxed toroidal states 59

15 Conclusion $\quad 61$

$\begin{array}{ll}\text { Part III } & 62\end{array}$

16 Adiabatic traps and mirror machines $\quad 62$

16.1 Digression on adiabatic invariants . . . . . . . . . . . . . 62

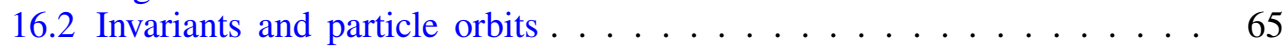

16.3 Orbit calculations . . . . . . . . . . . . . . . . . 67

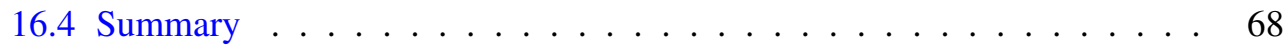

17 Simple mirror machines $\quad 68$

18 Equilibria in magnetic wells $\quad 72$

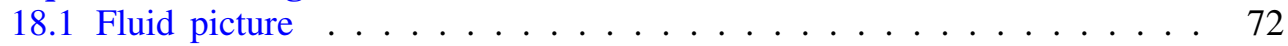

18.2 Particle picture . . . . . . . . . . . . . . . . . . . 74

18.3 Special minimum- $B$ equilibria . . . . . . . . . . . . . 74

19 Energy principles and stability $\quad \mathbf{7 5}$

19.1 Application to special minimum- $B$ equilibria . . . . . . . . . . 77

19.2 Direct proof of stability for special equilibria at low $\beta \ldots \ldots$

20 Canonical description of guiding centre motion $\quad 79$

21 General equilibria in mirror machines $\quad \mathbf{8 0}$

21.1 Stability of general equilibria in mirror machines at low $\beta \ldots$. . . 80 
22 Maximal ordering and stability $\quad \mathbf{8 2}$

22.1 Stability of general equilibria in mirror machines at finite $\beta \ldots$. . . 83

23 Conclusion $\quad 85$

$\begin{array}{ll}\text { Part IV } & 85\end{array}$

24 Ballooning modes $\quad \mathbf{8 5}$

24.1 Stability of plasma in circular cylinders . . . . . . . . . . . . 86

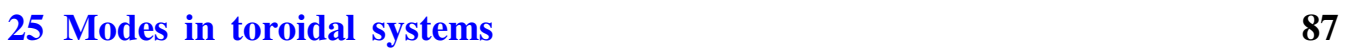

26 High mode number perturbations in an axisymmetric torus 88

26.1 Ballooning representation ................. 89

27 Magnetohydrodynamic stability in a torus $\quad 90$

27.1 Higher-order theory . . . . . . . . . . . . . . . . . . 94

28 Applications $\quad 95$

29 Validity of ballooning theory $\quad 98$

30 Mode structure $\quad 99$

31 Conclusion $\quad 100$

\section{Foreword}

The following notes are based on a course of lectures given by one of us (J.B.T.) at the University of Texas in Austin in 1991. As was the intention of this 'Special Topics' course, most of the lectures described the author's own work. Consequently, these notes are not intended as an introductory course in plasma physics. They assume some familiarity with the subject and, as lecture notes, they are less formal than a conventional text. We hope they will be useful as a supplement to a basic course, or for advanced students, or as a record of research in the pre-computer-simulation era.

Of course, the emphasis in plasma physics has changed dramatically since 1991. Owing to time constraints, we have not attempted to bring the material up to date, though some sections, notably those on adiabatic invariants and maximal ordering theory, have been expanded.

We are grateful to Emilia Solano and Phil Edmonds, who are largely responsible for preserving the original notes, and to A. Schekochihin for encouraging their publication.

\section{Part I}

\section{Introduction}

In these lectures we discuss several aspects of plasma confinement in magnetic fields. Such magnetised plasmas can exhibit complex behaviour. Even if the plasma is treated as a simple conducting fluid it can exhibit all the phenomena of an ordinary fluid, including turbulence, as well as electromagnetic phenomena such as wave propagation, scattering and refraction. More importantly, a magnetised plasma exhibits many new phenomena, arising from the interaction between the plasma and the magnetic field, and it is these new phenomena that are our main interest. 


\subsection{Long-range effects}

In reality a plasma is not a simple fluid. The idea of a simple fluid, with locally defined properties such as diffusivity, viscosity and thermal conductivity, depends on the fact that the mean free path of fluid particles is short. But in a high-temperature plasma the mean free paths of ions and electrons are usually much longer than the size of the system. Fortunately, in a magnetic field $\boldsymbol{B}$, the paths of ions and electrons are curled into tight spirals about the field, with (Larmor) radius $m_{s} v_{\perp} / e_{s} B$ - which is generally small. This tends to restore local behaviour transverse to $\boldsymbol{B}-$ but the long-range effect parallel to $\boldsymbol{B}$ persists. This indirectly influences cross-field transport, and the plasma may still not have unique local transport coefficients perpendicular to $\boldsymbol{B}$. (For example, the cross-field transport in a toroidal plasma depends on the global rotational transform.) Conversely, local changes in the magnetic field (e.g. field line reconnection) may lead to substantial changes in the global field structure. In a magnetised plasma 'local changes affect global properties and global changes affect local properties'.

So, in any plasma confinement problem, there are two aspects to be considered. The first is the introduction of a simplified model for the plasma; the second is the incorporation of this model into a realistic configuration for the confining field.

\subsection{Confinement systems}

Plasma confinement systems divide sharply into two classes, according to how one approaches the problem of loss due to flow of plasma along the magnetic field. In the first class, one accepts free flow parallel to $\boldsymbol{B}$, but ensures that the magnetic lines of force never leave the system. According to an important topological theorem of Poincaré, such a system can only be toroidal. (For a simple account of this, see Taylor (1974a).)

Alternatively, one may restrict the flow of plasma parallel to $\boldsymbol{B}$ by the 'magnetic mirror' effect (see $\S 16$ ). This depends on the adiabatic invariance of the magnetic moment $\mu=m v_{\perp}^{2} / B$ of a charged particle gyrating rapidly in a magnetic field. Because of this, a gyrating particle of energy $E$ moving along a field line into an increasing magnetic field is reflected when $B>2 E / \mu$. Unfortunately, this means that only particles outside the 'loss cone' $v_{\perp} / v<\left(B_{\min } / B_{\max }\right)^{1 / 2}$ in velocity space are reflected, and, as particles are continually scattered into this loss cone, there is always significant loss of plasma through magnetic mirrors.

\subsection{Plasma models}

\subsubsection{Fluid model}

The simplest model is the ideal conducting fluid:

$$
\begin{gathered}
\rho \frac{\mathrm{d} \boldsymbol{v}}{\mathrm{d} t}=\boldsymbol{j} \times \boldsymbol{B}-\nabla p, \\
\frac{1}{p} \frac{\mathrm{d} p}{\mathrm{~d} t}=\frac{\gamma}{\rho} \frac{\mathrm{d} \rho}{\mathrm{d} t}, \\
\frac{\partial \rho}{\partial t}+\nabla \cdot(\rho \boldsymbol{v})=0, \\
\nabla \times \boldsymbol{B}=\mu_{0} \boldsymbol{j}, \\
\nabla \cdot \boldsymbol{B}=0, \\
\nabla \times \boldsymbol{E}=-\frac{\partial \boldsymbol{B}}{\partial t}, \\
\boldsymbol{E}+\boldsymbol{v} \times \boldsymbol{B}=0 .
\end{gathered}
$$




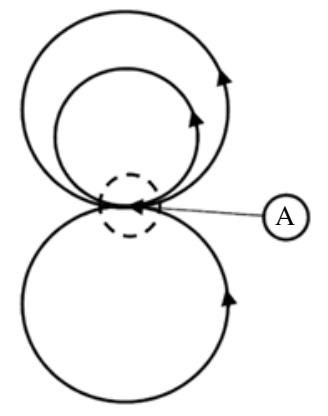

FIGURE 1. Fluid flow in the presence of a density gradient.

The key equation here is Ohm's law (1.7), which couples the magnetic field to the plasma motion. It represents the fact that, if plasma resistivity $\eta$ is neglected, the electric field must vanish in the plasma rest frame. Specifically it implies $\boldsymbol{E} \cdot \boldsymbol{B}=0$ and $\boldsymbol{v}_{\perp}=\boldsymbol{E} \times \boldsymbol{B} / B^{2}$.

More detailed fluid models include additional effects, such as resistivity, viscosity and so-called 'finite Larmor radius (FLR) effects'. (These are additional terms in the pressure tensor arising because particle orbits between collisions are spirals around the field lines rather than straight lines.)

Despite its simplicity and apparent deficiencies, this ideal fluid model represents large-scale plasma behaviour rather well, since the important features of anisotropy and plasma-field coupling are embodied in Ohm's law.

\subsubsection{Guiding centre model}

Another simple model is based on the notion of the 'guiding centre'. We noted that particle orbits in a plasma are tight spirals about a magnetic field line. A particle moves rapidly along this spiral but the spiral itself drifts slowly across the field. Two important such 'guiding centre drifts' are the electric ' $E$ cross $B$ ' drift,

$$
\boldsymbol{v}_{E}=\frac{\boldsymbol{E} \times \boldsymbol{B}}{B^{2}}
$$

and the magnetic ' $\operatorname{grad} B$ ' drift,

$$
\boldsymbol{v}_{B}=-\frac{m_{s}}{e_{s}} \frac{\nabla B \times \boldsymbol{B}}{B^{3}}\left(v_{\|}^{2}+\frac{v_{\perp}^{2}}{2}\right),
$$

where subscript $s$ denotes the plasma species. Note that the electric drift is identical for all particles, whilst the grad $B$ drift is different, and in the opposite direction, for ions and electrons. Note also that the guiding centre velocity and the fluid velocity are not necessarily the same. This can be seen from figure 1 . If there is a vertical density gradient, then even if the guiding centres are at rest $(\boldsymbol{E}=0)$, there is a non-zero fluid velocity at point $\mathrm{A}$ - because more orbits are centred above A than are centred below it.

\subsubsection{Kinetic theory models}

The most complete description of a plasma is in terms of a phase space distribution function $f_{s}(\boldsymbol{x}, \boldsymbol{v}, t)$ for each particle species,

$$
\frac{\partial f_{s}}{\partial t}+\boldsymbol{v} \cdot \nabla f_{s}+\frac{e_{s}}{m_{s}}\left(\boldsymbol{E}+\frac{1}{c} \boldsymbol{v} \times \boldsymbol{B}\right) \cdot \frac{\partial f_{s}}{\partial \boldsymbol{v}}=0 .
$$


The system is closed by coupling the fields to Maxwell equations through the charge and current source terms,

$$
\rho=\sum_{s} e_{s} \int \mathrm{d}^{3} v f_{s}(\boldsymbol{x}, \boldsymbol{v}, t), \quad \boldsymbol{j}=\sum_{s} e_{s} \int \mathrm{d}^{3} v \boldsymbol{v} f_{s}(\boldsymbol{x}, \boldsymbol{v}, t) .
$$

If the distribution function is interpreted as an assembly of discrete particles, $f \sim$ $\sum_{i} \delta\left(\boldsymbol{x}-\boldsymbol{x}_{i}(t)\right) \delta\left(\boldsymbol{v}-\boldsymbol{v}_{i}(t)\right)$, then (1.10) is exact (but pathological and not very useful) and is known as the 'Klimontovich equation'. Usually, $f(\boldsymbol{x}, \boldsymbol{v}, t)$ is interpreted as an ensemble average, or as smoothed over a small volume containing many particles. Then the particles are independent of one another (except for the long-range smoothed electric and magnetic fields) and (1.10) becomes the celebrated 'Vlasov equation'.

Mathematically, the smoothing referred to can be achieved by taking a limit in which the discrete plasma particles are subdivided into increasingly smaller units of mass and charge (i.e. $1 / n \rightarrow 0, e \rightarrow 0, m \rightarrow 0$ ) while maintaining $e / m$ and $n e$ constant, where $n$ is the species' density. This limit preserves the Debye length $\lambda_{D}=\sqrt{\epsilon_{0} T / n e^{2}}$ and the plasma frequency $\omega_{p}^{2}=n e^{2} / m$. In fact, the Vlasov equation can be regarded as the first approximation in a hierarchy of equations (similar to the BogoliubovBorn-Green-Kirkwood (BBGK) hierarchy in classical fluids) ordered in powers of a 'discreteness' parameter - essentially the inverse of the number of particles in a Debye sphere $1 / n \lambda_{D}^{3}$ (Davidson 1972; Nishikawa \& Wakatani 1993).

Note that, although the Klimontovich and Vlasov interpretations of (1.10) share the same equation, they are actually completely different. The Klimontovich equation involves tracking variables $(\boldsymbol{x}(t), \boldsymbol{v}(t))$ and initial values $(\boldsymbol{x}(0), \boldsymbol{v}(0))$, whereas the Vlasov equation involves a function $f(\boldsymbol{x}, \boldsymbol{v}, t)$ and initial condition $f\left(\boldsymbol{x}, \boldsymbol{v}, t_{0}\right)$.

Of course, this 'smoothing' eliminates the short-range interactions between particles, but these can be restored by introducing 'binary collisions' à la Boltzmann theory of neutral gases. Then,

$$
\frac{\partial f_{s}}{\partial t}+\boldsymbol{v} \cdot \nabla f_{s}+\frac{e_{s}}{m_{s}}\left(\boldsymbol{E}+\frac{1}{c} \boldsymbol{v} \times \boldsymbol{B}\right) \cdot \frac{\partial f_{s}}{\partial \boldsymbol{v}}=\sum_{s^{\prime}} C\left(f_{s}, f_{s^{\prime}}\right),
$$

where $C\left(f_{s}, f_{s^{\prime}}\right)$ is one of several 'collision operators'.

Actually, collisions in a plasma are different from those in a dilute gas. In a gas, collisions are infrequent 'close encounters' that scatter particles through large angles. In a plasma, these occasional large-angle scatterings are much less important than frequent small-angle scatterings due to 'distant encounters'. In fact, with a $1 / r^{2}$ Coulomb interaction, the total effect of the long-range encounters is infinite! This unfortunate feature is overcome by arguing that in a plasma the charge of each particle is screened by a cloud of oppositely charged particles attracted to it. This 'Debye' screening cloud reduces the force between particles to $\left(1 / r^{2}\right) \exp \left(-r / \lambda_{D}\right)$, where $\lambda_{D}=\sqrt{\epsilon_{0} T / n e^{2}}$ is the Debye length.

Although (1.12) is the most detailed description of a plasma, it often contains more detail than is needed. For example, one may be interested only in processes much slower than the cyclotron frequencies $(\omega \ll \Omega)$, or on a longer scale than the Larmor radius $(\rho \ll \lambda)$, or of small amplitude $(\delta f \ll f)$, and the Vlasov equation can be simplified accordingly. A very popular example of this is the 'gyrokinetic' model in which $\omega / \Omega \sim \rho / L \sim k_{\|} \rho \sim \delta f / f$, where $L$ is the plasma scale, but $k_{\perp} \rho \sim 1$ so that FLR effects are retained (Catto et al. 1981). 


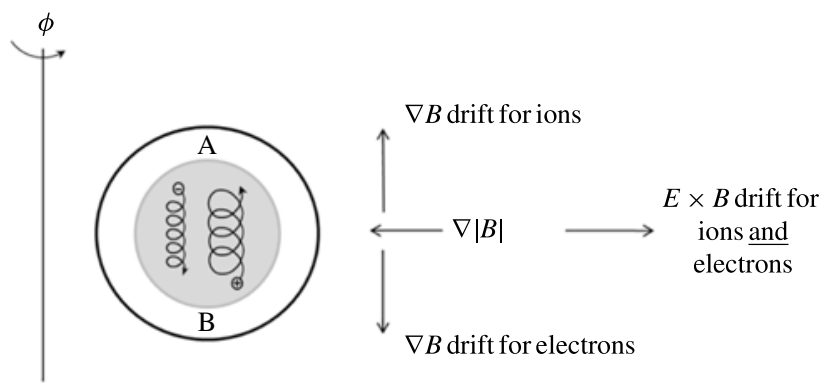

FIGURE 2. Particle motion in a toroidal field.

\section{Toroidal confinement}

It is interesting to show that there can be no containment of a static plasma in a purely toroidal, axisymmetric field. From the guiding centre viewpoint this is readily demonstrated. Consider plasma centred in the minor cross-section of a toroidal vacuum chamber (see figure 2). The $\operatorname{grad} B$ drift is opposite for electrons and ions, so that ions accumulate on the upper surface of the plasma at $A$ and electrons accumulate on the lower surface at $\mathrm{B}$. These charges create an electric field and the resulting $E \times B$ drift, which is the same for both ions and electrons, then causes the whole plasma to drift outwards away from the axis of symmetry.

The corresponding proof of this from the fluid model is longer - but more informative. Starting from the equilibrium force balance

$$
\boldsymbol{j} \times \boldsymbol{B}=\nabla p
$$

we have

$$
\boldsymbol{j}=\frac{\boldsymbol{B} \times \nabla p}{B^{2}}+\lambda \boldsymbol{B},
$$

where $\lambda$ is determined from $\nabla \cdot \boldsymbol{j}=0$,

$$
\begin{aligned}
B \frac{\mathrm{d} \lambda}{\mathrm{d} l} & =\boldsymbol{B} \cdot \nabla \lambda=-\nabla \cdot\left(\frac{\boldsymbol{B} \times \nabla p}{B^{2}}\right), \\
& =2 \frac{\boldsymbol{B} \times \nabla p \cdot \nabla B}{B^{3}}
\end{aligned}
$$

Integrating around the torus on a closed field line gives the constraint

$$
\oint\left(\frac{\boldsymbol{B} \times \nabla p \cdot \nabla B}{B^{4}}\right) \mathrm{d} l=0 .
$$

However, in a purely toroidal, axisymmetric, field, the integrand is independent of $l$, so equilibrium requires

$$
\boldsymbol{B} \times \nabla B \cdot \nabla p=0 .
$$

As illustrated in figure 3, the pressure $p$ would then have to be constant along lines parallel to the axis of symmetry of the torus and the plasma cannot therefore be contained! 


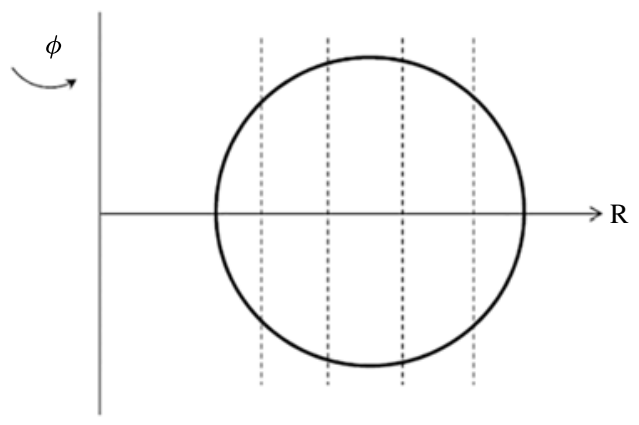

FIGURE 3. Constant pressure surfaces in a purely toroidal field.

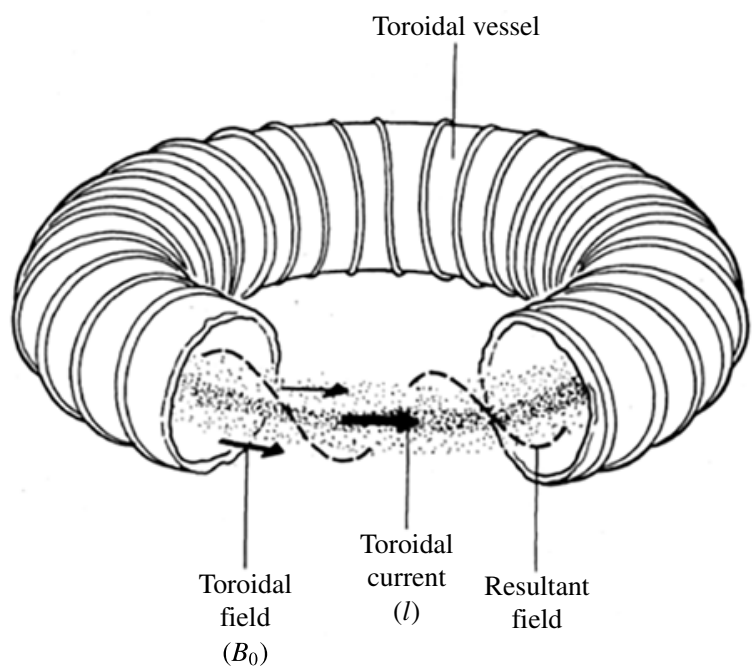

FIGURE 4. Geometry of the toroidal pinch (from Taylor 1986).

\subsection{Magnetic surfaces}

We can evade the constraint (2.4) if we prevent the lines of force closing after one circuit of the torus. A simple way to do this - which is the basis for the tokamak and the toroidal pinch - is to induce a toroidal current (see figure 4). This produces a poloidal field so that, after one circuit around the torus, a line of force is displaced poloidally from its starting point. Then (hopefully) if the line of force is followed indefinitely around the torus it will generate a closed 'toroidal magnetic surface' (or 'flux surface'), as shown in figure 5. The dots indicate successive intersections of a single field line with a plane at a fixed toroidal position, known as a Poincaré plot. The average angle, $\iota$, between successive intersections is the 'rotational transform' and the inverse of $\iota$ is the tokamak 'safety' factor, $q=2 \pi / \iota$. (This is also the ratio of the number of circuits a field line makes in the toroidal direction to the number it makes in the poloidal direction.)

Since $\boldsymbol{B} \cdot \nabla p=0$, we see that $p$ must be constant over a flux surface. Therefore, what we really require for confinement is a set of nested flux surfaces (see figure 6). 


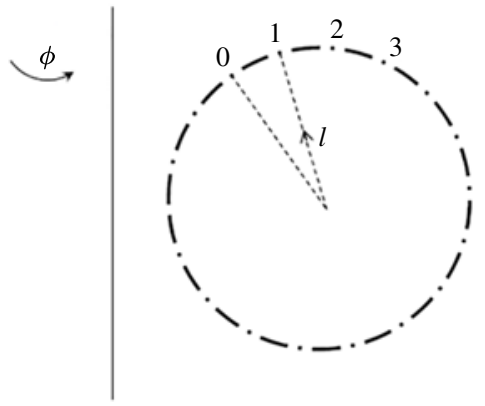

FIGURE 5. Poincaré plot generated by following a magnetic field line through many circuits around the torus.

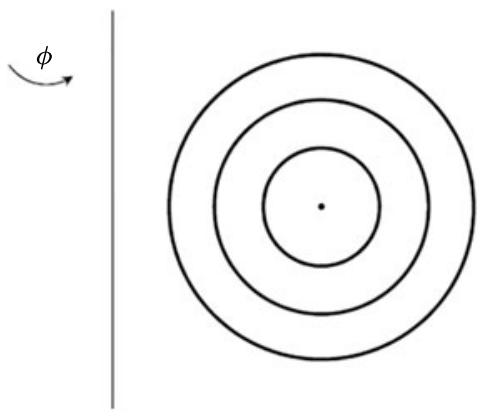

FIGURE 6. Nested magnetic flux surfaces.

Each magnetic surface is defined by $\Psi(x, y, z)=$ constant, where $\boldsymbol{B} \cdot \nabla \Psi=0$ and $p=p(\Psi)$. (The degenerate surface at the centre, where we want maximum plasma pressure, is the 'magnetic axis'.)

At this point we should consider what has become of the constraint (2.4) when we have flux surfaces rather than closed field lines. There is a very useful trick for dealing with a switch from closed lines of force to a closed toroidal flux surface. Consider an integral along a line of force such as

$$
\int X \frac{\mathrm{d} l}{B}
$$

Now introduce a thin flux tube of cross-section $\delta A$, with flux $\delta F=B \delta A$, enclosing this field line. Then

$$
\delta F \int X \frac{\mathrm{d} l}{B} \Rightarrow \int_{V} X \mathrm{~d} F \frac{\mathrm{d} l}{B} \Rightarrow \int_{V} X(B \mathrm{~d} A) \frac{\mathrm{d} l}{B} \Rightarrow \int_{V} X \mathrm{~d} \tau .
$$

Here the line integral has been converted to a volume integral. If the field line now sweeps out a surface, then the thin flux tube enclosing it sweeps out a thin shell between two adjacent toroidal flux surfaces, as shown in figure 7 . So we effectively replace

$$
\int \frac{X}{B} \mathrm{~d} l \Rightarrow \frac{\partial}{\partial \Psi} \int_{V} X \mathrm{~d} \tau
$$

where $V(\Psi)$ is now the volume within a toroidal flux surface. 


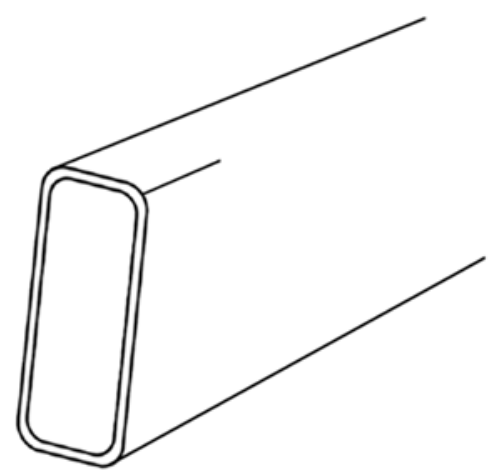

FIGURE 7. Shell swept out by thin flux tube surrounding flux surface.

Applying this result to the constraint (2.4), it becomes

$$
\frac{\partial}{\partial \Psi} \int \nabla \cdot\left(\frac{\boldsymbol{B} \times \nabla p}{B^{2}}\right) \mathrm{d} \tau=\frac{\partial}{\partial \Psi} \oint\left(\frac{\boldsymbol{B} \times \nabla p}{B^{2}}\right) \cdot \mathrm{d} \boldsymbol{S}=0
$$

which is automatically satisfied since $\nabla p$ is normal to the toroidal flux surface (i.e. parallel to $\mathrm{d} \boldsymbol{S}$ ).

We should also see what becomes of the constraint (2.4) when the magnetic field is non-axisymmetric. For this we write the vector potential (as we can any vector) as $\boldsymbol{A}=\alpha \times \nabla \beta+\nabla \phi$, so $\boldsymbol{B}=\nabla \alpha \times \nabla \beta$. The functions $\alpha(x, y, z)$ and $\beta(x, y, z)$, known as Clebsch variables, are constant along a field line and the pressure is a function $p=p(\alpha, \beta)$. Then we have

$$
\boldsymbol{B} \cdot \nabla p \times \nabla B=\left(\frac{\partial p}{\partial \alpha} \frac{\partial B}{\partial \beta}-\frac{\partial p}{\partial \beta} \frac{\partial B}{\partial \alpha}\right) B^{2}
$$

and (2.4) becomes

$$
\oint\left(\frac{\partial p}{\partial \alpha} \frac{\partial B}{\partial \beta}-\frac{\partial p}{\partial \beta} \frac{\partial B}{\partial \alpha}\right) \frac{\mathrm{d} l}{B^{2}}=0 .
$$

Remembering that $\alpha$ and $\beta$ are constant along $\mathrm{d} l$, this implies

$$
\left(\frac{\partial p}{\partial \alpha} \frac{\partial U}{\partial \beta}-\frac{\partial p}{\partial \beta} \frac{\partial U}{\partial \alpha}\right)=0
$$

where

$$
U \equiv \oint \frac{\mathrm{d} l}{B}
$$

Hence, in a toroidal system with closed, but not necessarily purely toroidal, field lines, equation (2.4) imposes the constraint $p \equiv p(U)$.

In conclusion, therefore, the consequences of the equilibrium force balance $\boldsymbol{j} \times \boldsymbol{B}=$ $\nabla p$ in a torus are:

(i) If field lines generate flux surfaces $\Psi$, then $p=p(\Psi)$.

(ii) If field lines close, $p$ is constant along $\boldsymbol{B}$ and $p=p(U)$, where $U=\oint \mathrm{d} l / B$. 
This means that, in a non-axisymmetric torus, there is a potential conflict between the constraints $p=p(\Psi)$ and $p=p(U)$ at the so-called 'rational surfaces' where the rotational transform $\iota=2 \pi m / n$ with $m$ and $n$ integers and the lines of force close after $n$ circuits. Mathematically, these rational surfaces have zero measure compared to the irrational surfaces. Obviously, the simple fluid model is inadequate to discuss such fine details. Nevertheless, low-order rational surfaces (where $m$ and $n$ are small) play an important role in the stability of toroidal plasmas.

\subsection{Magnetic surfaces and the Grad-Shafranov equation}

We saw that for good containment we need an equilibrium with closed toroidal magnetic surfaces. But do solutions of $\boldsymbol{j} \times \boldsymbol{B}=\nabla p$ with closed magnetic surfaces really exist? In general this is a difficult question, related to the Kolmogorov-Arnold-Moser (KAM) theorem and mathematical chaos, and further complicated by the conflict mentioned above. However, solutions do exist in an axisymmetric torus and in other configurations with an ignorable coordinate (Grad \& Rubin 1958).

\subsubsection{Cylindrical}

The simplest magnetic surfaces are those in a straight cylinder where, in cylindrical coordinates $(r, \theta, z)$, the field is independent of $z$ and we can therefore write it as

$$
\boldsymbol{B}=\boldsymbol{n}_{z} \times \nabla \Psi(r, \theta)+\boldsymbol{n}_{z} f(r, \theta),
$$

where $\boldsymbol{n}_{z}$ is a unit vector in the $z$ direction. Then $\boldsymbol{\nabla} \cdot \boldsymbol{B}=0$ and $\boldsymbol{B} \cdot \boldsymbol{\nabla} \Psi=0$, so if $\Psi$ exists it is a magnetic surface. To find the surface $\Psi$, we start from the equilibrium $\boldsymbol{j} \times \boldsymbol{B}=\nabla p$. Then we know that $p$ must be a function of $\Psi$. Using the expression (2.14) for the field, the current is

$$
\boldsymbol{j}=\boldsymbol{n}_{z} \nabla^{2} \Psi-\boldsymbol{n}_{z} \times \nabla f
$$

so that for equilibrium $\Psi$ must satisfy

$$
\left(\nabla^{2} \Psi\right) \nabla \Psi+\left(\boldsymbol{n}_{z} \times \nabla f \cdot \nabla \Psi\right) \boldsymbol{n}_{z}+f \nabla f+p^{\prime} \nabla \Psi=0 .
$$

The component of (2.16) parallel to $\boldsymbol{n}_{z}$ requires

$$
\boldsymbol{n}_{z} \times \nabla f \cdot \nabla \Psi=0
$$

so that $f$ must also be a function only of $\Psi$. The component of (2.16) perpendicular to $\boldsymbol{n}_{z}$ then gives an equation for $\Psi$,

$$
\frac{1}{r} \frac{\partial}{\partial r} r \frac{\partial \Psi}{\partial r}+\frac{1}{r^{2}} \frac{\partial^{2} \Psi}{\partial \theta^{2}}+p^{\prime}(\Psi)+f f^{\prime}(\Psi)=0 .
$$

This is known as the Grad-Shafranov equation ${ }^{\dagger}$ for equilibrium in a cylinder - a two-dimensional partial differential equation. Solutions can be found once reasonable pressure and flux functions, $p(\Psi)$ and $f(\Psi)$, and boundary conditions, are specified.

\footnotetext{
$\dagger$ This equation was also introduced independently by other authors in the 1950 s when there were isolated national fusion programmes.
} 


\subsubsection{Toroidal}

Of course, an infinite cylinder is not very practicable, but it illustrates how to proceed in more realistic cases. A very important case is the axisymmetric torus an idealisation of a tokamak or toroidal pinch. In this case the field is independent of $\phi$ in $(r, \phi, z)$ coordinates and we can write it as

$$
\boldsymbol{B}=\frac{1}{r}\left[\boldsymbol{n}_{\phi} \times \nabla \psi(r, z)+\boldsymbol{n}_{\phi} F(r, z)\right]
$$

and the current is

$$
\boldsymbol{j}=-\frac{1}{r}\left[\boldsymbol{n}_{\phi} \times \nabla\left(r B_{\phi}\right) \times+\boldsymbol{n}_{\phi} \Delta^{*} \Psi\right]
$$

where $\Delta^{*}=r^{2} \nabla \cdot\left(r^{-2} \nabla \Psi\right)$. As in the cylindrical case, equilibrium then requires $p=p(\Psi)$ and $F=F(\Psi)$ and the equation for equilibrium in an axisymmetric torus becomes

$$
r \frac{\partial}{\partial r} \frac{1}{r} \frac{\partial \Psi}{\partial r}+\frac{\partial^{2} \Psi}{\partial z^{2}}+r^{2} p^{\prime}(\Psi)+F F^{\prime}(\Psi)=0 .
$$

This is again a two-dimensional partial differential equation with solutions specified by two arbitrary functions $p(\Psi)$ and $F(\Psi)$ and appropriate boundary conditions. A simple example is given below.

\subsubsection{An example}

A simple example of toroidal equilibrium is given by (2.21), with

$$
\begin{gathered}
p=8\left(1+\alpha^{2}\right) \Psi \\
F=\text { constant }
\end{gathered}
$$

The equilibrium equation then becomes

$$
r \frac{\partial}{\partial r}\left(\frac{1}{r} \frac{\partial \Psi}{\partial r}\right)+\frac{\partial^{2} \Psi}{\partial z^{2}}+8 r^{2}\left(1+\alpha^{2}\right)=0,
$$

with a solution

$$
\Psi=r^{2}\left(2 R^{2}-r^{2}-4 \alpha^{2} z^{2}\right) .
$$

In this case, the magnetic surfaces are toroids of approximately elliptical cross-section (see figure 8), surrounding the magnetic axis at $r=R$ and bearing some resemblance to the shape of modern tokamaks.

\subsubsection{Helical}

There is a similar equation to (2.21) for an equilibrium with helical symmetry (Freidberg 2014). This represents a 'straight' version of a stellarator (see figure 9), where the field in $(r, \theta, z)$ coordinates depends only on $r$ and $u=(l \theta+k z)$. This helical problem can be treated in a similar way to the cylindrical and toroidal problems by introducing a vector

$$
\boldsymbol{h}=\frac{\left(\boldsymbol{l n}_{z}-k r \boldsymbol{n}_{\theta}\right)}{\left(l^{2}+k^{2} r^{2}\right)^{1 / 2}} .
$$




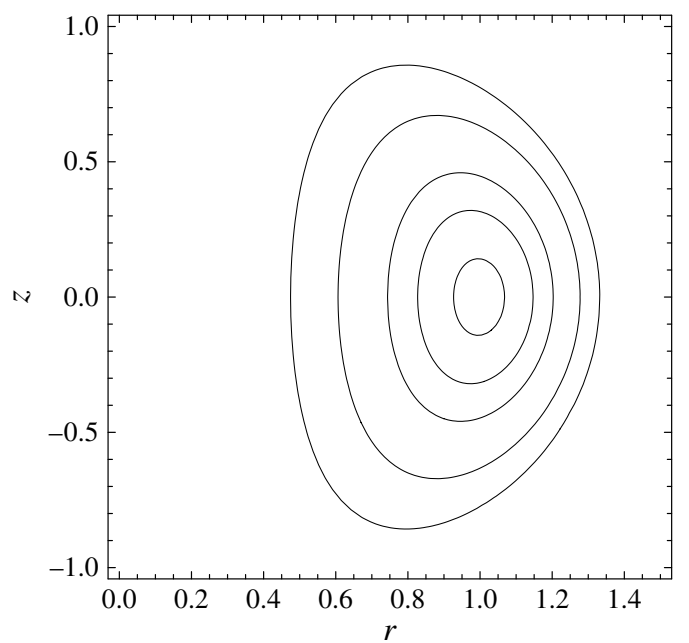

FIgURE 8. Cross-section of magnetic surfaces defined by (2.25), for $R=1 \mathrm{~m}$ and $\alpha=0.5$.

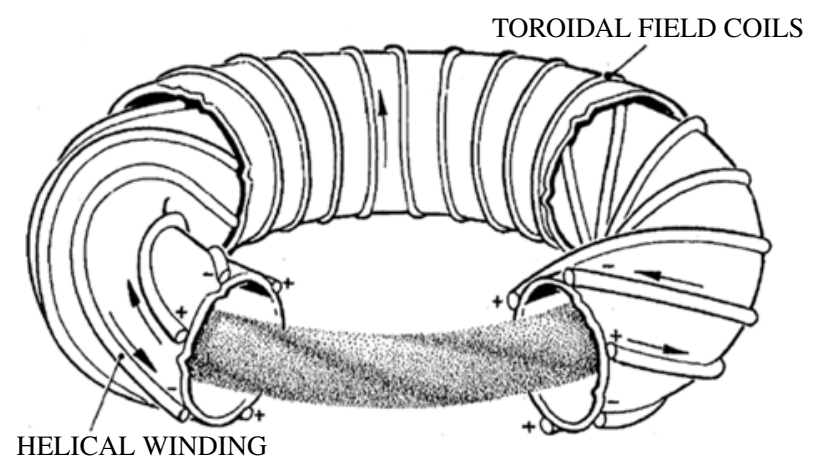

FIGURE 9. Sketch of a stellarator configuration.

(Note that $\boldsymbol{n}_{z}, \boldsymbol{n}_{\theta}$ and $\boldsymbol{h}$ are all unit vectors.) Then the field can be written as

$$
\boldsymbol{B}=\frac{1}{\left(l^{2}+k^{2} r^{2}\right)^{1 / 2}}[\boldsymbol{h} \times \nabla \Psi(r, u)+\boldsymbol{h} F(r, u)]
$$

and, after some algebra, $F$ and $p$ are again found to be functions only of $\Psi$, and the equation for helical equilibrium is

$$
\frac{1}{r} \frac{\partial}{\partial r} \frac{r}{\left(l^{2}+k^{2} r^{2}\right)} \frac{\partial \Psi}{\partial r}+\frac{1}{r^{2}} \frac{\partial^{2} \Psi}{\partial u^{2}}+p^{\prime}(\Psi)+\frac{F F^{\prime}(\Psi)}{\left(l^{2}+k^{2} r^{2}\right)}-\frac{2 k l F(\Psi)}{\left(l^{2}+k^{2} r^{2}\right)^{2}}=0 .
$$

(It is important in this case not to confuse the fixed pitch $k$ of the helical symmetry, imposed by boundary conditions, with the pitch of the lines of force - which varies from flux surface to flux surface; see §3.)

The three cases described above comprise the only exact magnetohydrodynamic (MHD) equilibria and only the toroidal case represents a realistic situation (the others extend to infinity). 


\subsection{Rotational transform}

An important property of a toroidal magnetic surface is its rotational transform $\iota(\Psi)$, or safety factor $q=2 \pi / \iota$. Indeed, a rotational transform is essential for toroidal confinement (see $\$ 2.1$ ). In general,

$$
\frac{\iota(\Psi)}{2 \pi} \equiv \lim \left(\frac{\text { number of circuits made by line of force in poloidal direction }}{\text { number of circuits made in toroidal direction }}\right),
$$

where the limit is as the number of circuits $\rightarrow \infty$. The simplest configuration in which a rotational transform appears is a circular cylinder, with periodicity $2 \pi R$, to simulate a torus. In this case each field line is a uniform spiral about the $z$ axis, $r \mathrm{~d} \theta / \mathrm{d} z=$ $B_{\theta} / B_{z}$. The magnetic surfaces are circular cylinders and

$$
\frac{\iota(\Psi)}{2 \pi}=\frac{B_{\theta} R}{r B_{z}}=\frac{I_{z}}{\psi_{z}},
$$

where $I_{z}$ and $\psi_{z}$ are the current and flux within the surface. Note that this does not depend on the presence of plasma - the current $I_{z}$ may as well flow in a thin wire along the $z$ axis.

Of course, this is trivial; everyone knows an axial current generates an encircling field! What is not trivial is that if we add an externally generated helical field (to resemble a straight stellarator) then a rotational transform exists without any axial current!

One can be forgiven if initially this appears to contradict Ampere's law, for if we take a circular loop integral around the $z$ axis, $\oint r B_{\theta} \mathrm{d} \theta$ must vanish when there is no axial current. This paradox is resolved if we note that the helical field modulates $B_{\theta}$, so that a loop round a flux surface has fluctuating $r$ and $B_{\theta}$. To first order these fluctuations cancel, but a second-order effect remains.

A detailed calculation (Johnson et al. 1958; Spitzer 1958) shows that a helical field $\sim B_{\text {hel }} \cos (l \theta+k z)$ generates a rotational transform $\iota \sim r^{2 l-4}\left(B_{h e l}^{2} / B_{t o r}^{2}\right)\left[2(l-1)+k^{2} r^{2}\right]$ near the magnetic axis. The transform therefore differs according to whether $l=1$ (when it vanishes on the axis and increases slowly with minor radius $r$ ), $l=2$ (when the transform is finite near the axis and increases slowly with $r$ ) or $l \geqslant 3$ (when the transform vanishes on the axis and increases rapidly with $r$ ). These differences are important for plasma stability and $l=3$ is usually a preferred value - because shear in the rotational transform generally enhances stability.

Surprisingly, neither axial current nor helical windings are necessary for a rotational transform! Indeed, the original suggestion for generating a rotational transform (Spitzer 1951) was to take a simple toroidal solenoid, with no additional currents or fields, and to 'twist' it into a figure-of-eight (see figure 10). In fact, any solenoidal field with a twisted magnetic axis generates a rotational transform. This is purely a geometrical effect and $\iota$ is the integral of the torsion around the (closed) magnetic axis, independent of minor radius.

\section{Perturbations of symmetry}

We have seen that, at least in principle, flux surfaces exist in systems with an ignorable coordinate. Now let us look in more detail at the effect of perturbations of this symmetry. Consider the field in a section of an infinite circular cylinder (of 


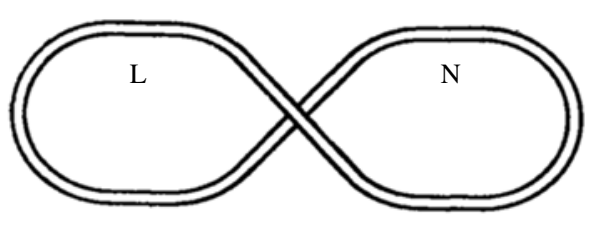

$\mathrm{M}$

K

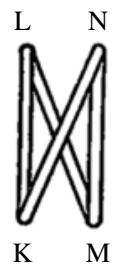

FIGURE 10. Sketch of figure-eight stellarator. (Reproduced with permission from Spitzer (1958). Copyright 1958, AIP Publishing LLC.)

length $2 \pi R$ to represent toroidal periodicity). In coordinates $(r, \theta, z)$ this has symmetry in both $\theta$ and $z$. The magnetic field has components $B_{r}=0, B_{\theta}=B_{\theta}(r)$ and $B_{z}=B_{z}(r)$, creating cylindrical flux surfaces $\Psi(r)$ with rotational transform (over the $2 \pi R$ section) $\iota / 2 \pi=1 / q(\Psi)=B_{\theta} R / r B_{z}$.

Any perturbation of this cylindrical field can be decomposed into helical components $\sim \exp [\mathrm{i}(m \theta+k z)]$, where $k=-n / 2 \pi R$. We first examine the effect of a single such helical component. Then the combined field has helical symmetry. As we mentioned earlier, the pitch of this helical symmetry is in general different from the pitch of the unperturbed field lines, but on a 'resonant surface', where $m-n q(\Psi)=0$, the helical perturbation and the unperturbed field lines have the same pitch.

As in $\$ 2.2 .4$, the combined field can be written as

$$
\boldsymbol{B}=\frac{1}{\left(m^{2}+k^{2} r^{2}\right)^{1 / 2}}[\boldsymbol{h} \times \nabla \Psi(r, u)+\boldsymbol{h} F(r, u)],
$$

where $u=(m \theta+k z)$ and $\boldsymbol{h}$ is the unit vector

$$
\boldsymbol{h}=\frac{\left(\boldsymbol{l n}_{z}-k r \boldsymbol{n}_{\theta}\right)}{\left(l^{2}+k^{2} r^{2}\right)^{1 / 2}} .
$$

The flux surfaces $\Psi$ of the combined field will have the form

$$
\Psi=\Psi_{0}(r)+\psi(r) \cos (m \theta+k z)
$$

and we can expect the effect of the helical perturbation to be important near a resonant surface, when the perturbation is constant along the unperturbed field (otherwise the effect of the perturbation is largely averaged out). From (3.1) the radius $r_{0}$ of the resonant surface is determined by $\partial \Psi_{0} / \partial r=0$ and near this surface we can write (3.3) as

$$
\Psi=\frac{1}{2} \Psi_{0}^{\prime \prime}\left(r-r_{0}\right)^{2}+\psi \cos (m \theta+k z) .
$$

The cross-sections of the corresponding surfaces, at fixed $z$, are shown in figure 11 for perturbations with $m=3$ and $m=7$ symmetry. As expected, most of the original surfaces are only slightly deformed by the perturbation, but the resonant surface is broken up to form a chain of 'islands'. The width of an island can be estimated from (3.4) as

$$
\Delta=4\left[\frac{\psi}{\Psi_{0}^{\prime \prime}\left(r_{0}\right)}\right]^{1 / 2}
$$


(a)

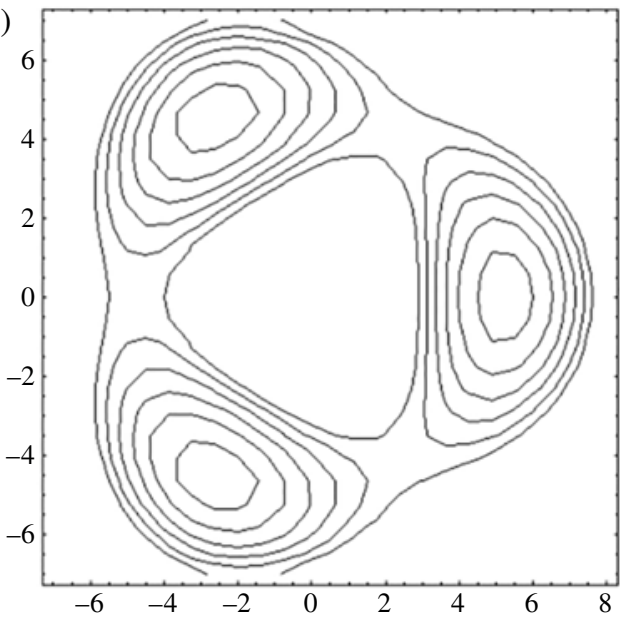

(b)

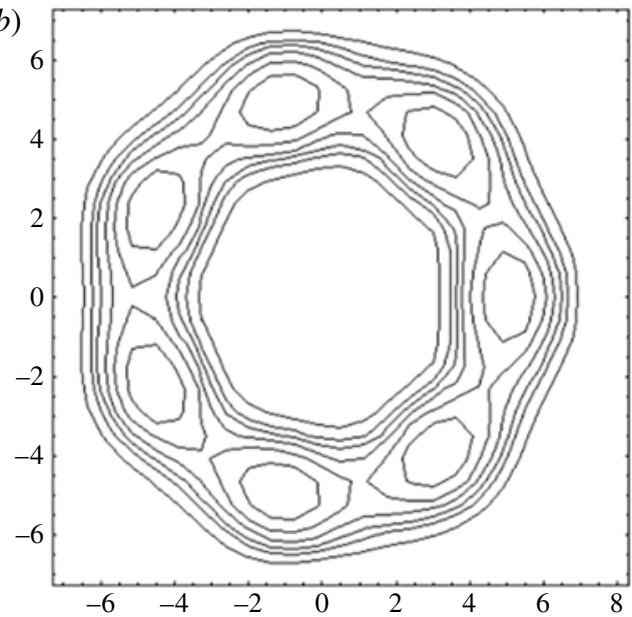

FIGURE 11. Magnetic surfaces resulting from a helical perturbation with $(a) m=3$ and (b) $m=7$.

A more practical form of this result is obtained using (3.1), which shows $\delta B_{r} \sim$ $\partial \Psi / \partial \theta \sim m \psi / r$ and $\Psi_{0}^{\prime} \sim r B_{z} / R q$, so

$$
\Delta \sim 4\left(\frac{\delta B_{r}}{B_{z} q^{\prime}}\right)^{1 / 2}\left(\frac{q^{2} R}{m}\right)^{1 / 2} .
$$

Thus the island width is proportional to the square root of the perturbing field, and inversely proportional to the square root of the shear $q^{\prime}$.

\subsection{Chaotic regions}

We have seen that a single helical perturbation of circular surfaces produces islands at a resonant surface, but these are still magnetic surfaces, albeit more complex ones. This is because the perturbed system retains an ignorable coordinate $(m \theta+k z)$. Note that this is true only of the straight helical system; it is not true if we add a helical field to a torus (as in a toroidal stellarator!).

When we impose two or more perturbations of different helical pitch there is no longer an ignorable coordinate (Rosenbluth et al. 1966). If the amplitudes of the two perturbations are small, numerical computations show that each perturbation produces a separate chain of islands at its own resonant surface - but the interaction between them produces small chaotic regions near the X-points of each chain. As the perturbations increase, these chaotic regions expand to fill the space between the two chains.

This behaviour is illustrated in a plane slab model with two incommensurable Fourier perturbations (Wesson 2004). The fields in this model are

$$
\begin{gathered}
B_{z}=B_{0}=\text { constant } \\
B_{x}=B_{x}^{\prime} y, \\
B_{y}=\delta B_{y}\left[\cos \left(k_{z} z+k_{x} x\right)+\cos \left(k_{z} z-k_{x} x\right)\right],
\end{gathered}
$$


(a)

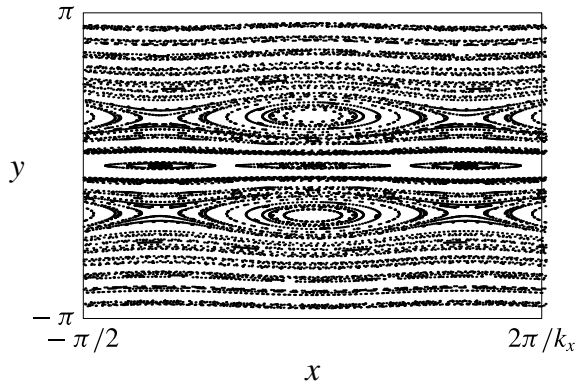

(c)

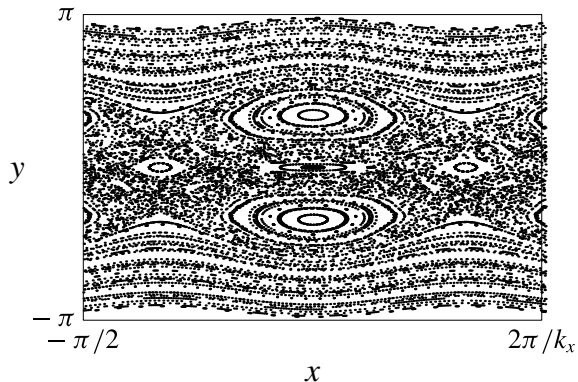

(b)

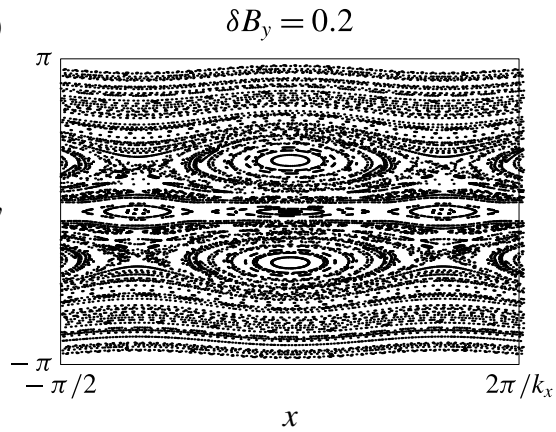

(d)

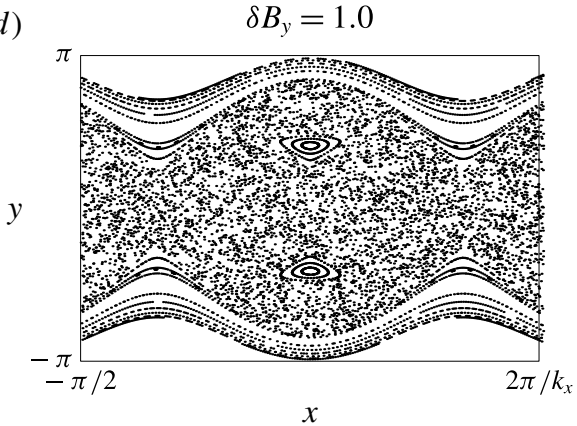

FIGURE 12. Illustration of the break-up of magnetic surfaces in the presence of multiple perturbations with increasing strength: (a) $\delta B_{y}=0.1$; (b) $\delta B_{y}=0.2 ;$ (c) $\delta B_{y}=0.3$; (d) $\delta B_{y}=1.0$.

and the field lines are determined by integrating

$$
\frac{\mathrm{d} x}{\mathrm{~d} z}=\frac{B_{x}}{B_{z}}, \quad \frac{\mathrm{d} y}{\mathrm{~d} z}=\frac{B_{y}}{B_{z}} .
$$

The resulting Poincaré plots at fixed $z$ are shown in figure 12. As each chaotic zone is generated by following a single field line, plasma can readily flow throughout a chaotic zone. An interesting question is therefore: What is the magnitude of the perturbations that cause the chaotic zones to first extend from one island chain to the next? This can be estimated by calculating the width of the islands in each chain, according to the formula given above, and assuming that the last magnetic surface between island chains disappears when their individual widths overlap. However, as we remarked earlier, an accurate calculation is a fundamental problem in mathematical chaos theory.

\subsection{Mappings}

One way to study chaotic break-up of surfaces is by numerical calculation of field lines and construction of the Poincaré plot as in the figures above. However, as field lines must be followed very accurately for many circuits of the torus, such calculations are lengthy. Therefore one often uses a simple 'Iterative Mapping' to illustrate the break-up of magnetic surfaces (Lichtenberg \& Lieberman 2010).

The mapping

$$
\begin{gathered}
x_{n+1}=f\left(x_{n}, y_{n}\right), \\
y_{n+1}=g\left(x_{n}, y_{n}\right),
\end{gathered}
$$


simulates successive intersections of a field line with the Poincare surface of section without consideration of the path between intersections. Such mappings can easily be rapidly and accurately iterated many times.

In order to represent $\boldsymbol{\nabla} \cdot \boldsymbol{B}=0$, the mapping must be area-preserving. That is, any area must map into an equal area. A general area-preserving map can be written implicitly in the form

$$
\begin{gathered}
x_{n+1}=x_{n}-\frac{\partial G}{\partial \theta}, \\
\theta_{n+1}=\theta_{n}+\frac{\partial G}{\partial x_{n+1}},
\end{gathered}
$$

where $G \equiv G\left(x_{n+1}, \theta_{n}\right)$.

The simplest map that simulates the formation of magnetic islands and chaotic regions is the standard or Chirikov-Taylor map (Lichtenberg \& Lieberman 2010),

$$
\begin{gathered}
x_{n+1}=x_{n}+k \sin \theta_{n}, \\
\theta_{n+1}=\theta_{n}+x_{n}+k \sin \theta_{n} .
\end{gathered}
$$

This corresponds to

$$
G\left(x_{n+1}, \theta_{n}\right)=\frac{x_{n+1}^{2}}{2}+k \cos \theta_{n} .
$$

The important features of this mapping are:

(i) it is periodic in $\theta$, representing the poloidal angle;

(ii) when $k=0, x_{n}=$ constant, representing the behaviour of a line of force lying on a magnetic surface;

(iii) the difference $\left(\theta_{n+1}-\theta_{n}\right)$ represents the rotational transform $\iota$ on a flux surface (note that this varies from one surface to another); and

(iv) the term $k \sin \theta$ represents a perturbation of the magnetic field.

In a Poincaré plot generated by a continuous field line between intersections of the Poincaré plane, $k \sin \theta$ would represent perturbation by a single helical mode. As we have seen, a single helical perturbation does not create chaotic regions, but because the mapping is discontinuous, the term $k \sin \theta$ is equivalent to a discontinuous perturbation of the field containing many helical modes.

The results of iterating the standard map are shown in figure 13 for several values of the perturbation amplitude $k$. As expected, when $k$ is small, islands appear with small chaotic regions near X-points. These chaotic regions expand, at the expense of continuous surfaces, as $k$ increases until the last continuous surface between island chains disappears. By the overlap criterion above, this occurs at $k_{c} \sim \pi^{2} / 4$; the true value is $0.971635 .$. (Greene 1968; Lichtenberg \& Lieberman 2010).

\section{Motion of lines of force}

So far we have considered only stationary plasmas and fields. We now turn to some aspects of the motion of magnetic fields and plasmas. This depends on the time scale of the motion and the resistivity of the plasma. As expected, the key lies in Ohm's law, which connects the magnetic field with the plasma motion and with resistivity. 
(a)

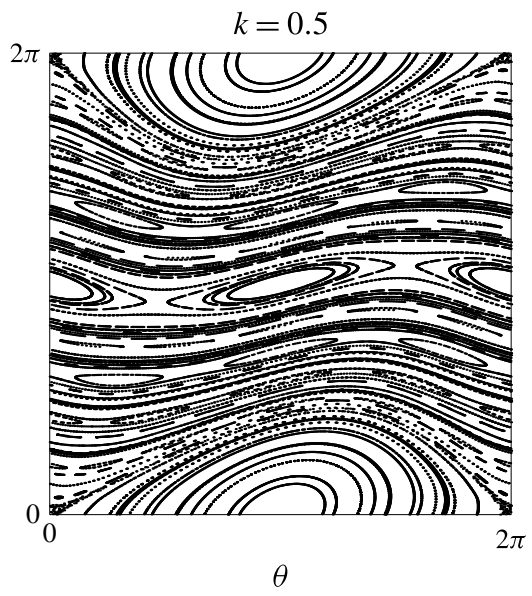

(c)

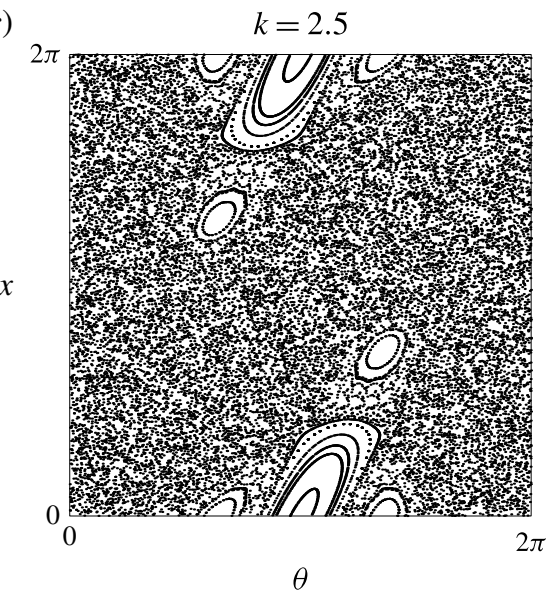

(b)

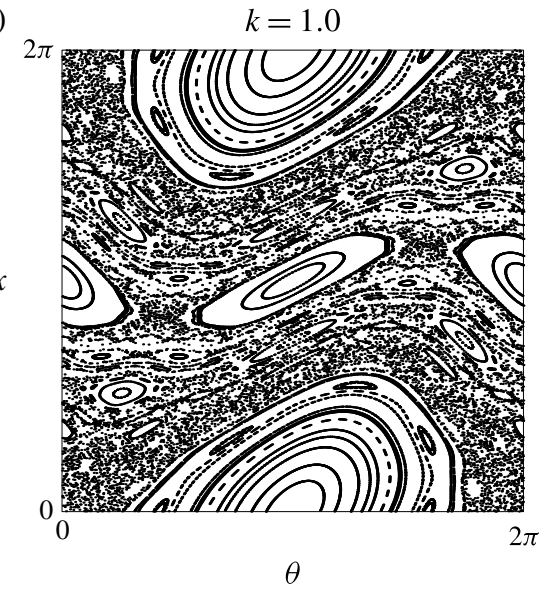

(d)

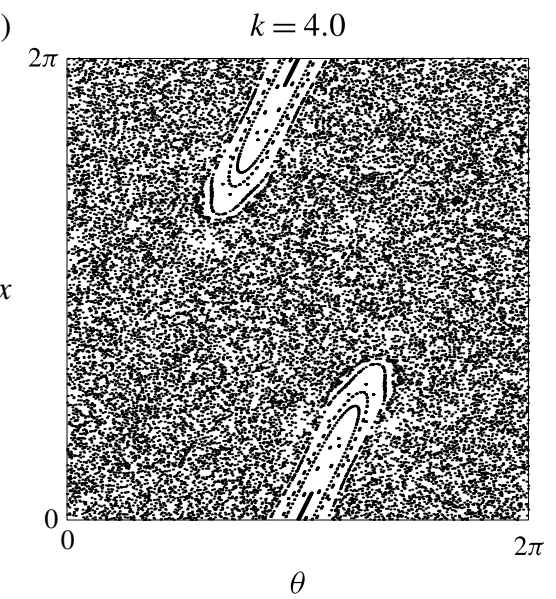

FIGURE 13. Result of the standard map (3.15)-(3.16) for varying $k$ : (a) $k=0.5$; (b) $k=1.0 ;$ (c) $k=2.5 ;(d) k=4.0$.

\subsection{Fluid model with resistivity}

The resistive fluid model is described by

$$
\left.\begin{array}{c}
\rho \frac{\mathrm{d} \boldsymbol{v}}{\mathrm{d} t}=\boldsymbol{j} \times \boldsymbol{B}-\nabla p, \\
\frac{1 \mathrm{~d} p}{p} \frac{\gamma}{\mathrm{d} t}=\frac{\mathrm{d} \rho}{\rho}, \\
\frac{\partial \rho}{\partial t}+\nabla \cdot(\rho \boldsymbol{v})=0, \\
\nabla \times \boldsymbol{B}=\mu_{0} \boldsymbol{j}, \\
\nabla \cdot \boldsymbol{B}=0, \\
\nabla \times \boldsymbol{E}=-\frac{\partial \boldsymbol{B}}{\partial t}, \\
\boldsymbol{E}+\boldsymbol{v} \times \boldsymbol{B}=\eta \boldsymbol{j},
\end{array}\right\}
$$


and the evolution of the field is given by

$$
\frac{\partial \boldsymbol{B}}{\partial t}=\nabla \times(\boldsymbol{v} \times \boldsymbol{B})+\frac{\eta}{\mu_{0}} \nabla^{2} \boldsymbol{B} .
$$

The two important time scales in a plasma can be seen in the behaviour of smallamplitude waves, transverse to $\boldsymbol{B}_{0}$ in an incompressible plasma (Alfvén waves). Then $b \sim \exp (-\mathrm{i} \omega t+\mathrm{i} \boldsymbol{k} \cdot \boldsymbol{r})$ and (4.1) reduce to

$$
\begin{gathered}
-\mathrm{i} \omega \boldsymbol{b}_{\perp}=\mathrm{i} \boldsymbol{k} \times\left(\boldsymbol{v}_{\perp} \times \boldsymbol{B}_{0}\right)+\frac{\eta}{\mu_{0}} \boldsymbol{k} \times\left(\boldsymbol{k} \times \boldsymbol{b}_{\perp}\right)=\mathrm{i}\left(\boldsymbol{k} \cdot \boldsymbol{B}_{0}\right) \boldsymbol{v}_{\perp}-\frac{\eta}{\mu_{0}} k^{2} \boldsymbol{b}_{\perp}, \\
-\mathrm{i} \omega \rho_{0} \boldsymbol{v}_{\perp}=\mu_{0}^{-1}\left(\mathrm{i} \boldsymbol{k} \times \boldsymbol{b}_{\perp}\right) \times \boldsymbol{B}_{0} \approx \mathrm{i} \mu_{0}^{-1}\left(\boldsymbol{k} \cdot \boldsymbol{B}_{0}\right) \boldsymbol{b}_{\perp},
\end{gathered}
$$

giving

$$
\omega^{2} \boldsymbol{v}_{\perp}=\left[\frac{\left(\boldsymbol{k} \cdot \boldsymbol{B}_{0}\right)^{2}}{\mu_{0} \rho_{0}}-\mathrm{i} \omega \frac{\eta}{\mu_{0}} k^{2}\right] \boldsymbol{v}_{\perp} .
$$

Equation (4.5) defines the characteristic speed $v_{A}^{2}=B_{0}^{2} / \mu_{0} \rho_{0}$ of Alfvén waves, the Alfvén time scale $\tau_{A}^{2} \sim a^{2} \mu_{0} \rho_{0} / B_{0}^{2}$ and the resistive time scale of the wave damping $\tau_{R} \sim \mu_{0} a^{2} / \eta$.

The ratio of the resistive to Alfvén time scales is the Lundquist number, $S$,

$$
S=\frac{\tau_{R}}{\tau_{A}} \sim \frac{a^{2}}{\eta} \frac{B}{a \sqrt{\mu_{0} \rho}} .
$$

In high-temperature laboratory plasmas $S \sim 10^{6}-10^{8}$ (in astrophysical plasmas it is $\sim 10^{14}$ ). These large values of $S$ create serious difficulties but can also lead to great simplification. The difficulties lie in computing over such an enormous range of time scales. The simplification is that field diffusion can often be ignored and the resistivity set to zero. This leads to some crucial properties of an ideal plasma, or of a real plasma on a time scale much less than $\tau_{R}$.

(i) Flux conservation. The magnetic flux $\phi=\int_{S} \boldsymbol{B} \cdot \mathrm{d} \boldsymbol{A}$ through an area $S$ bounded by a closed curve $C$ that moves with the fluid is constant.

The rate of change of the flux is

$$
\frac{\mathrm{d} \phi}{\mathrm{d} t}=\int \frac{\partial \boldsymbol{B}}{\partial t} \cdot \mathrm{d} \boldsymbol{A}+\oint \boldsymbol{B} \cdot \boldsymbol{v} \times \mathrm{d} \boldsymbol{l},
$$

where the first term is due to the change in the field $\boldsymbol{B}$ and the second is due to motion of the boundary moving with the fluid. The total change can be written as

$$
\int\left[\frac{\partial \boldsymbol{B}}{\partial t}-\nabla \times(\boldsymbol{v} \times \boldsymbol{B})\right] \cdot \mathrm{d} \boldsymbol{A},
$$

which, by virtue of the field evolution equation (4.2), is always zero when $\eta=0$.

(ii) Field line preservation. A line moving with the fluid that is initially a line of force always remains a line of force. (Alternatively, if fluid particles initially lie on a common line of force then they continue to do so.)

To show this, it is convenient to reintroduce Clebsch variables as in $\S 2.1$. Then

$$
\boldsymbol{B}=\nabla \alpha \times \nabla \beta,
$$




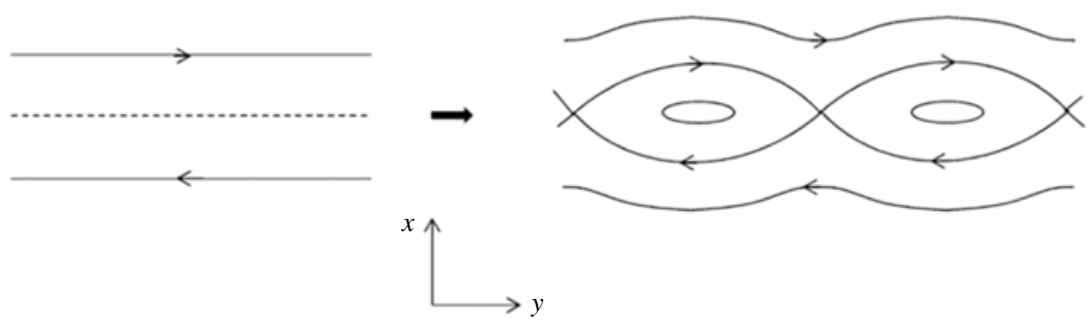

FIGURE 14. Magnetic island formation.

where $\alpha(x, y, t)$ and $\beta(x, y, t)$ are constant along a line of force (crucially they label each line of force). Therefore

$$
\frac{\partial \boldsymbol{B}}{\partial t}=\left(\nabla \frac{\partial \alpha}{\partial t}\right) \times \nabla \beta-\left(\nabla \frac{\partial \beta}{\partial t}\right) \times \nabla \alpha .
$$

However, we also have

$$
\begin{aligned}
\frac{\partial \boldsymbol{B}}{\partial t} & =\nabla \times[\boldsymbol{v} \times(\nabla \alpha \times \nabla \beta)] \\
& =-\nabla \times[(\boldsymbol{v} \cdot \nabla \alpha) \nabla \beta-(\boldsymbol{v} \cdot \nabla \beta) \nabla \alpha] \\
& =-\nabla(\boldsymbol{v} \cdot \nabla \alpha) \times \nabla \beta+\nabla(\boldsymbol{v} \cdot \nabla \beta) \times \nabla \alpha,
\end{aligned}
$$

where $\boldsymbol{v}$ is the fluid velocity. Although (4.10) and (4.11) do not completely determine $\partial_{t} \alpha$ and $\partial_{t} \beta$, a consistent solution is

$$
\frac{\partial \alpha}{\partial t}+\boldsymbol{v} \cdot \nabla \alpha=0, \quad \frac{\partial \beta}{\partial t}+\boldsymbol{v} \cdot \nabla \beta=0 .
$$

That is to say, there is a labelling of the lines of force in a moving ideal plasma such that each line moves with the fluid velocity $\boldsymbol{v}_{F}=\boldsymbol{E} \times \boldsymbol{B} / B^{2}$. Because the field lines are locked into the fluid, they cannot break or intersect one another, since this would require a discontinuous fluid velocity.

Note that the choice of field line labels, and of their velocity, is not unique. In fact, instead of saying that field lines move with the velocity of the fluid, $\boldsymbol{v}$, we could equally say that they move with velocity $(\boldsymbol{v}+\boldsymbol{u})$, where $\boldsymbol{u} \times \boldsymbol{B}=\nabla G$, and $G$ is any function satisfying $\boldsymbol{B} \cdot \nabla G=0$. Of course, no physical quantity would be affected by this. It would simply be a different labelling of the imaginary lines of force.

\subsection{Effect of field line preservation}

The effect of field line preservation in an ideal plasma can be seen if one tries to calculate the growth of magnetic islands in the so-called 'sheared slab' configuration $\boldsymbol{B}_{0}=(0, s x B, B)$, shown in figure 14. In this configuration $B_{y}$ varies only in the $x$ direction and changes sign at $x=0$. The question is: Can the field lines in this equilibrium reconnect across the $x=0$ plane to form magnetic islands? This would occur if there were an unstable perturbation with $B_{x 1} \neq 0$ at $x=0$. For example, a single Fourier mode $\sim \exp (\mathrm{i} k y)$ could create a chain of islands like those in figure 14 . (For such a mode, the 'resonant surface' $\boldsymbol{k} \cdot \boldsymbol{B}_{0}=0$ is located at $x=0$.) 
A perturbation $\left(\boldsymbol{B}_{1}, \boldsymbol{v}_{1}\right)$ of the sheared slab equilibrium satisfies

$$
\frac{\partial \boldsymbol{B}_{1}}{\partial t}=\left(\boldsymbol{B}_{0} \cdot \nabla\right) \boldsymbol{v}_{1}-\left(\boldsymbol{v}_{1} \cdot \boldsymbol{\nabla}\right) \boldsymbol{B}_{0}-\boldsymbol{B}_{0}\left(\boldsymbol{\nabla} \cdot \boldsymbol{v}_{1}\right)
$$

and for the single Fourier mode $B_{1} \sim B_{1}(x) \exp (\mathrm{iky})$,

$$
\frac{\partial B_{x 1}}{\partial t}=\left(\mathrm{i} s k x B_{0}\right) v_{x 1} .
$$

We see from this that growth of a reconnecting field across the $x=0$ plane would require an infinite fluid velocity at $x=0$ if the plasma resistivity were precisely zero!

\section{Resistive plasmas}

In a resistive plasma, field lines can break and reconnect. At first sight we might expect this process to be slow, as it is in resistive diffusion. However, reconnection occurs much more rapidly if it is concentrated in a small region $\delta \ll a$. Then it occurs at a rate $\eta / \delta^{2} \gg 1 / \tau_{R}$. An important example of this rapid reconnection occurs in resistive instabilities.

\subsection{Resistive instabilities: the tearing mode}

The basic 'tearing mode' instability involves the growth of magnetic islands in the sheared slab that we considered in the previous section (Furth et al. 1963). As we found, if $\eta=0$ any such growth is prevented by the field line preservation constraint, but now we wish to calculate the rate of growth when $\eta \neq 0$. In particular, we are interested in instabilities that grow rapidly compared to resistive diffusion, but slowly compared to MHD Alfvén time scales.

We again start from a sheared slab equilibrium $\boldsymbol{v}=0$ and $\boldsymbol{B}_{0}=\left(0, B_{0 y}(s x), B_{0 z}\right)$. We assume that $B_{0 y}(s x)$ is symmetric about $x=0$ and that the plasma is incompressible. Then the growth of a linear perturbation $\left(\boldsymbol{B}_{1}, \boldsymbol{v}\right)$ is given by

$$
\frac{\partial \boldsymbol{B}_{1}}{\partial t}=\boldsymbol{B}_{0} \cdot \nabla \boldsymbol{v}-\boldsymbol{v} \cdot \nabla \boldsymbol{B}_{0}+\frac{\eta}{\mu_{0}} \nabla^{2} \boldsymbol{B}_{1}
$$

and

$$
\rho_{0} \frac{\partial \boldsymbol{v}}{\partial t}=-\nabla p+\boldsymbol{j}_{1} \times \boldsymbol{B}_{0}+\boldsymbol{j}_{0} \times \boldsymbol{B}_{1} .
$$

It is convenient to write $\boldsymbol{B}_{1}=\hat{z} \times \nabla A_{1}$. Then for a Fourier mode $A \sim A_{1}(x) \exp (\gamma t+$ $\mathrm{i} k y), B_{x 1}=-\mathrm{i} k A_{1}$, and we can extract equations for $\left(A_{1}, v_{x}\right)$ alone, decoupled from all other components,

$$
-\gamma A_{1}-B_{0 y}(s x) v_{x} B_{0}+\frac{\eta}{\mu_{0}} \nabla_{\perp}^{2} A_{1}=0
$$

and

$$
\frac{\gamma \rho_{0}}{k^{2}} \nabla_{\perp}^{2} v_{x}-\frac{B_{0 y}(s x)}{\mu_{0}} \nabla_{\perp}^{2} A_{1}+\frac{\mathrm{d} j_{0}}{\mathrm{~d} x} A_{1}=0 .
$$

We saw that, when $\eta=0$, island growth requires $v_{x} \rightarrow \infty$ at $x=0$. Hence we expect the effect of resistivity to be most important near $x=0$. We therefore divide the calculation into a thin inner layer around $x=0$, of thickness $\delta$, where resistivity is important, and two outer regions $|x|>\delta$, where resistivity can be neglected. A solution is then constructed in each outer region and matched to the solution in the inner layer. 
Because we expect the tearing mode to grow slowly compared to the Alfvén time, we can neglect inertia in the outer regions. Then in these regions

$$
\frac{\mathrm{d}^{2} A^{e x t}}{\mathrm{~d} x^{2}}-k^{2} A^{e x t}-\frac{\mu_{0}}{B_{0 y}(s x)} \frac{\mathrm{d} j_{0}}{\mathrm{~d} x} A^{e x t}=0 .
$$

Note that this is exactly the equation for ideal plasma equilibrium in the outer regions - this equilibrium merely adjusts itself to the slowly changing inner solution.

We can integrate (5.5) inwards from \pm infinity, or from the plasma boundaries, and adjust the (linear) amplitude of the left and right solutions to be equal at $x=0$. Then in general we will find that there is a discontinuity in the slope $(\mathrm{d} A / \mathrm{d} x)$ between the left and right solutions at $x=0$. We define this discontinuity as the 'stability index',

$$
\Delta^{\prime} \equiv \frac{1}{A(0)}\left[\left(\frac{\mathrm{d} A}{\mathrm{~d} x}\right)_{+\delta}-\left(\frac{\mathrm{d} A}{\mathrm{~d} x}\right)_{-\delta}\right] .
$$

This $\Delta^{\prime}$ is all we require from the outer solutions and we will see later that $\Delta^{\prime}>0$ implies instability.

We now need to find a solution in the inner region. In this region $x$ is small but $v_{x}$ and $A_{1}$ vary very rapidly with $x$. We can therefore neglect the last term in (5.4) as well as derivatives in the $y$ direction. Then, in the thin resistive inner layer, equations (5.3) and (5.4) reduce to

$$
\begin{gathered}
\frac{\mathrm{d}^{2} A}{\mathrm{~d} x^{2}}-\frac{\gamma \mu_{0}}{\eta} A-\frac{s B_{0} \mu_{0}}{\eta} x v_{x}=0, \\
\frac{\gamma \rho_{0}}{k^{2}} \frac{\mathrm{d}^{2} v_{x}}{\mathrm{~d} x^{2}}-\frac{s^{2} B_{0}^{2}}{\eta} x^{2} v_{x}-\frac{s B_{0} \gamma}{\eta} x A=0 .
\end{gathered}
$$

We now 'stretch' the thickness of the inner layer by introducing $\tau=x / \delta$, and select

$$
\delta^{4}=\frac{\eta \gamma \rho_{0}}{s^{2} k^{2} B_{0}^{2}}
$$

so that the layer has unit width in $\tau$. We also rescale the velocity by introducing $w=\left(s B_{0} \delta / \gamma\right) v_{x}$. Then the resistive layer equations become

$$
\begin{gathered}
\frac{\mathrm{d}^{2} w}{\mathrm{~d} \tau^{2}}-\tau^{2} w=\tau A, \\
\frac{\mathrm{d}^{2} A}{\mathrm{~d} \tau^{2}}=\frac{\gamma \mu_{0} \delta^{2}}{\eta}(A+\tau w) .
\end{gathered}
$$

This essentially completes the solution! The change in $\mathrm{d} A / \mathrm{d} \tau$ across the inner layer will be of order $\gamma \mu_{0} \delta^{2} / \eta$. Hence, when we match the change in $\mathrm{d} A / \mathrm{d} x$ to the discontinuity $\Delta^{\prime}$ in the outer solutions, we will have

$$
\Delta^{\prime}=\frac{\gamma \mu_{0} \delta}{\eta} \times(\text { number of order unity })
$$

Therefore $\Delta^{\prime}=0$ is the stability threshold and, when $\Delta^{\prime}>0$,

$$
\gamma \sim \frac{\eta^{3 / 5}}{\mu_{0}^{4 / 5}}\left(\frac{s k B_{0}}{\rho^{1 / 2}}\right)^{2 / 5}\left(\Delta^{\prime}\right)^{4 / 5}
$$


In terms of Alfvén and resistive times, $\tau_{A}=a \sqrt{\mu_{0} \rho} / B_{0}$ and $\tau_{R}=a^{2} \mu_{0} / \eta$, defined relative to a characteristic dimension $a$ of the outer plasma, equation (5.13) becomes

$$
\gamma \sim \frac{\left(a \Delta^{\prime}\right)^{4 / 5}(k a)^{2 / 5}(s a)^{2 / 5}}{\tau_{R}^{3 / 5} \tau_{A}^{2 / 5}} .
$$

Consequently $\gamma \tau_{R} \sim S^{2 / 5} \gg 1$ and $\gamma \tau_{A} \sim S^{-3 / 5} \ll 1$. So our assumptions about the tearing mode are confirmed: when resistivity is small, the growth rate is indeed rapid compared to resistive diffusion but slow compared to the Alfvén speed, and the resistive layer is thin, with the width $\delta$ given by (5.9). Note that in the limit $\eta \rightarrow 0$ we recover an ideal plasma, but with a discontinuity in the form of a current sheet at the resonant layer $\boldsymbol{k} \cdot \boldsymbol{B}_{0}=0-$ a possibility we did not consider in our discussion of ideal plasmas.

It is important to recognise the significance of tearing modes. Of course, we expect that introducing a small resistivity will perturb the motion of a previously ideal plasma. But this is not what tearing modes do. Tearing modes are new modes involving critical layers in which resistivity is dominant no matter how small it may be. Mathematically they arise because the differential equations governing resistive plasma are of higher order than those governing ideal plasma. This means that there are solutions for the resistive plasma that do not exist when resistivity is strictly zero. (This is very similar to the effect of viscosity in fluid dynamics, where it also introduces additional solutions and is dominant in critical boundary layers.)

In addition to its intrinsic interest, there are several reasons why the calculation of tearing modes is important: $(a)$ it describes an instability that has been seen in experiments, $(b)$ it illustrates the importance of small departures from ideal Ohm's law, $(c)$ it confirms that reconnection can occur much faster than resistive diffusion and $(d)$ the matching procedure described in the calculation is used in many other calculations when there is a small departure from the ideal plasma. In these calculations more complicated models are used in the resonant layer, but the outer region calculation is essentially unchanged.

In one respect, however, our calculation of the tearing mode growth rate is atypical. Recall that in the outer region

$$
\frac{\mathrm{d}^{2} A}{\mathrm{~d} x^{2}}-k^{2} A=\frac{\mu_{0}}{B_{0 y}(s x)} \frac{\mathrm{d} j_{0}}{\mathrm{~d} x} A .
$$

We assumed that $j_{0}$ was symmetric about $x=0$, so the right-hand side of this equation tends to a constant times $A$ as $x \rightarrow 0$ and the general solution near $x=0$ is

$$
A \sim \alpha[1+\cdots]+\beta[x+\cdots] .
$$

In this case $\mathrm{d} A / \mathrm{d} x$ and $A$ are well behaved at $x=0$ and there is no difficulty in calculating $\Delta^{\prime}$. But if $j_{0}$ is not symmetric about $x=0$, the right-hand side of the equation $\sim A / x$ as $x \rightarrow 0$. Then the general solution near $x=0$ is

$$
A \sim \alpha[1+c(x \log |x|-x)]+\beta[x+\cdots],
$$

so that $A$ and $\mathrm{d} A / \mathrm{d} x$ are divergent at $x=0$. In this case, rather than matching the derivatives of inner and outer solutions, we match the large $(\alpha)$ and small $(\beta)$ solutions in the inner and outer regions. (If we had included plasma pressure in our calculation, 

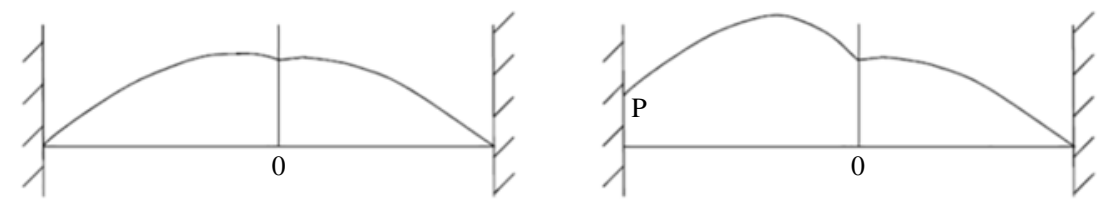

FIGURE 15. Faster reconnection: $P$ represents the forcing perturbation at the wall.

the general solution would have been of the form $\alpha|x|^{q}+\beta|x|^{1-q}$ near $x=0$, but we again match the large and small solutions.)

One might reasonably question whether at this level of detail the plasma can really be represented by a fluid model. However, the underlying concept of reconnection occurring in a thin layer, but dependent on a parameter $\Delta^{\prime}$ calculated from the plasma outside this layer, appears to be robust. This view is reinforced by another interpretation of $\Delta^{\prime}$. Suppose we remove the resistive layer and replace it by a thin flexible insulating membrane. The magnetic pressure on this membrane is $B^{2} / 2 \mu_{0}$, so if we deform it by a tearing-mode-like displacement $\xi_{x}$, the work done by the plasma (per unit area) on one side of the membrane is

$$
\delta W \sim \xi_{x}\left(\frac{B_{0}^{2}}{2}+\boldsymbol{B}_{0} \cdot \boldsymbol{B}_{1}\right) .
$$

As there is no change of plasma volume, the contribution from $B_{0}^{2}$ is zero, and from the earlier calculation of tearing modes, in the ideal plasma

$$
\begin{gathered}
\boldsymbol{B}_{0} \cdot \boldsymbol{B}_{1}=B_{0 y}(s x) \frac{\partial A}{\partial x} \\
\xi_{x} B_{0 y}(s x)=-A
\end{gathered}
$$

where $\gamma \xi_{x}=v_{x}$. Therefore the net change of energy is

$$
\delta W \sim A(0)^{2}\left[\left(\frac{1}{A} \frac{\mathrm{d} A}{\mathrm{~d} x}\right)_{-\varepsilon}-\left(\frac{1}{A} \frac{\mathrm{d} A}{\mathrm{~d} x}\right)_{\varepsilon}\right] .
$$

Hence $\Delta^{\prime}$ is a measure of the energy available in the ideal plasma if the field line conservation constraint is relaxed at the resonant surface $\boldsymbol{k} \cdot \boldsymbol{B}_{0}=0$.

\subsection{Forced reconnection}

As we saw, tearing mode reconnection is driven by the energy released from an unstable plasma. However, reconnection may occur even more rapidly when it is 'forced', that is, driven by plasma flows or imposed on the reconnecting region. Conceptually we might view the forcing as being applied externally, as in figure 15, effectively increasing the value of $\Delta^{\prime}$. In practice we will be more interested in reconnection driven by turbulence.

An often quoted idealised form of forced reconnection is the Sweet-Parker model. This represents plasma flow through an X-point of a two-dimensional magnetic field, as illustrated in figure 16. The antiparallel reconnecting fields are forced towards the $\mathrm{X}$-point by flows in the $\pm x$ direction, reconnect, and then flow outwards along the $\pm y$ directions. We can readily imagine that the forced inflows cause 'flattening' 


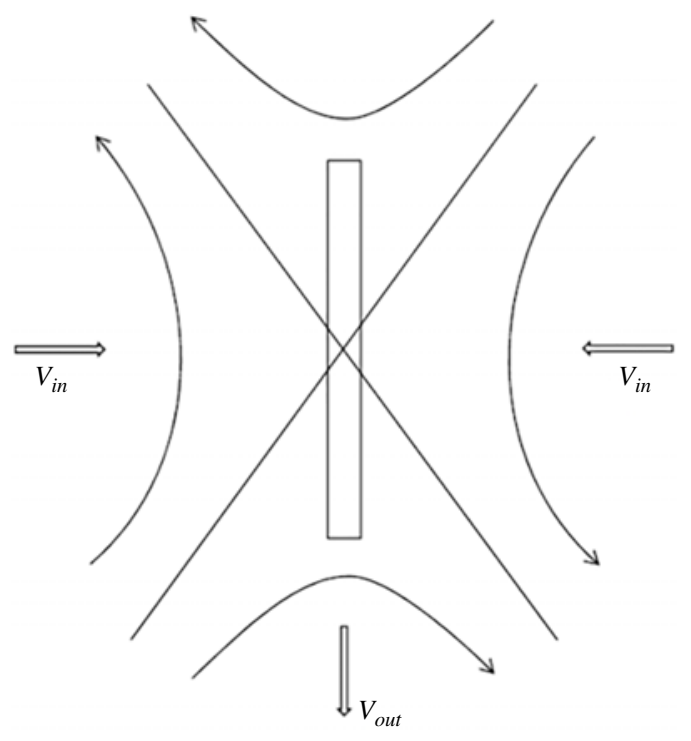

FIGURE 16. Model of forced reconnection.

of the X-point to eventually form a current sheet of thickness $\delta$ and length $l$, as indicated in figure 16, across which the field $B$ changes direction. We can then envisage a steady state, with plasma forced into the current sheet across the field, at a velocity $v_{x, \text { in }}$ controlled by resistivity, and flowing out along the field at a much higher velocity, $v_{y, \text { out }}$.

From Ohm's law we have for the inflow, $v_{i n} \sim \eta / \delta \mu_{0}$. Pressure balance, $\rho v_{y}^{2} / 2=$ $B_{0}^{2} / 2 \mu_{0}$, gives for the outflow, $v_{\text {out }} \sim B / \sqrt{\mu_{0} \rho}$, and mass conservation requires that $v_{\text {out }} \delta \sim v_{\text {in }} l$. Consequently, $v_{\text {in }}^{2} \sim l^{2} /\left(\tau_{R} \tau_{A}\right)$ so that flux is swept in, and reconnects at a rate $\sim\left(\tau_{A} \tau_{R}\right)^{-1 / 2}$, where $\tau_{A}$ and $\tau_{R}$ are again the Alfvén and resistive diffusion times relative to the outer dimensions of the reconnecting region (with scale length $\sim l$ ).

Of course, both the tearing mode and the Sweet-Parker model are over-idealised and, except in carefully controlled experiments, can scarcely represent real situations. In particular, the strictly two-dimensional nature of these models is likely to be unrepresentative, and the Sweet-Parker outflow is also likely to be unstable. (Numerical simulations generally show that reconnection is faster than it is in the idealised models.)

In a turbulent plasma, a more realistic picture is one in which elements of plasma, carrying differently oriented frozen-in fields, are forced together. In the absence of reconnection, they could not penetrate each other and a current layer would form between them. However, resistivity allows some penetration and reconnection. If this is not fast enough to keep up with the inflow, magnetic field will pile up against the current sheet, making the Alfvén speed larger and the current sheet thinner, so that reconnection speeds up. Consequently, in a turbulent resistive plasma, we can expect reconnection to proceed freely.

\section{Conclusion}

In Part I we have introduced some important basic features of magnetised plasmas, including the strong anisotropy (short range of effects in the direction perpendicular 
to $\boldsymbol{B}$, long range parallel to $\boldsymbol{B}$ ) and the consequent importance of magnetic field lines and surfaces. Their most important properties are:

(i) In an ideal $(\eta=0)$ plasma, there are many constraints on their motion arising from the fact that field lines cannot break or reconnect - even though they are hypothetical! These constraints dominate the behaviour of ideal plasmas.

(ii) In an even slightly resistive plasma these constraints may be ineffective, leading to new phenomena (as well as small modifications of the ideal behaviour). In turbulent resistive plasmas we expect breaking and reconnection of field lines to be the rule rather than the exception, and, as we will see in Part II, this provides an explanation and a prediction of the behaviour of turbulent plasmas in a very wide range of experiments.

\section{Part II}

\section{Plasma relaxation}

Plasma relaxation theory (Taylor $1974 b, 1975,1986,2000$ ) has its origin in early experiments on toroidal pinches - notably ZETA (Butt et al. 1966; Gibson et al. 1967). In these early pinch experiments, a toroidal field $B_{0}$ is first created by external coils and a toroidal current $I$ is then induced in the plasma (essentially by a transformer in which the plasma torus is the secondary). This current rapidly heats and compresses the plasma. A remarkable feature of these experiments is that, after an initial, violently turbulent phase, the plasma enters a 'quiescent' phase in which the turbulence is much reduced. Even more remarkable is the fact that in the quiescent phase the plasma profile is almost independent of any details of the initial state and depends principally on the 'pinch ratio' $\theta=B_{\theta} / B_{0}$, where $B_{\theta}$ is the poloidal field at the plasma boundary. (Alternatively, $\theta=2 I / a B_{0}$, where $a$ is the torus minor radius.) Furthermore, when $\theta$ exceeds a critical value, which is the same for all quiescent states, the toroidal field in the outer plasma is spontaneously reversed relative to the initial applied field!

These observations suggest that the plasma has reached some preferred, possibly stable, state - now known as the 'relaxed state'. The idea of such a state can be illustrated by a simple analogy. Suppose we immerse a flexible current-carrying loop of wire in a viscous insulating medium. What is its equilibrium state? While the wire is moving, it loses energy to the viscous medium, so its equilibrium must be a state of minimum energy - but minimum energy subject to certain constraints. (Otherwise all the energy would be dissipated!) One constraint is that the flux $L I$ linking the loop ( $L$ is the inductance) is constant, and if this were the only constraint the equilibrium would be the configuration of minimum $L I^{2} / 2$, subject to $L I$ being constant. (This is the state of maximum inductance.)

A plasma resembles an infinity of linked flexible wires and the first problem is to identify the appropriate constraints (Taylor 1986).

\subsection{Relaxation constraints}

We have seen that, for a perfectly conducting plasma, important constraints follow from

$$
\frac{\partial \boldsymbol{B}}{\partial t}=\nabla \times(\boldsymbol{v} \times \boldsymbol{B}),
$$

which we now write as

$$
\frac{\partial \boldsymbol{A}}{\partial t}=\boldsymbol{v} \times \boldsymbol{B}+\nabla \chi
$$


where $\boldsymbol{A}$ is the vector potential and $\chi$ is an arbitrary scalar. Consequently, changes in $\boldsymbol{A}$ must satisfy a similar equation,

$$
\delta \boldsymbol{A}=\boldsymbol{\xi} \times \boldsymbol{B}+\nabla \bar{\chi},
$$

where $\xi$ is an arbitrary plasma displacement (and $\bar{\chi}$ follows from $\chi$ ). It is clear that this does not restrict $\delta \boldsymbol{A}_{\perp}$, but $\delta A_{\|}$is constrained by

$$
\oint \frac{\mathrm{d} l}{B} \boldsymbol{B} \cdot \delta \boldsymbol{A}=0
$$

for any closed field line or magnetic surface (see $§ 2.1$ ).

A more convenient, and more fundamental, form of this constraint is that, for every infinitesimal flux tube surrounding a closed field line labelled by Clebsch variables $\alpha$, $\beta$, and for every magnetic flux surface, the volume integral

$$
K_{\alpha \beta} \equiv \int_{\alpha, \beta} \boldsymbol{A} \cdot \boldsymbol{B} \mathrm{d} \tau
$$

is invariant. This provides an infinity of constraints - which replace the single constraint of the flexible wire.

We can confirm the invariance of $K_{\alpha \beta}$ directly:

$$
\frac{\mathrm{d} K_{\alpha \beta}}{\mathrm{d} t}=\int_{V} \boldsymbol{B} \cdot \frac{\partial \boldsymbol{A}}{\partial t} \mathrm{~d} \tau+\int_{V} \boldsymbol{A} \cdot \frac{\partial \boldsymbol{B}}{\partial t} \mathrm{~d} \tau+\oint_{S}(\boldsymbol{A} \cdot \boldsymbol{B}) \boldsymbol{v} \cdot \mathrm{d} \boldsymbol{S},
$$

where $V$ and $S$ are respectively the volume and surface area of the infinitesimal flux tube. The last term arises from the motion of the flux tube and the others from the local change in $(\boldsymbol{A} \cdot \boldsymbol{B})$. Then, from (7.2),

$$
\int_{V} \boldsymbol{B} \cdot \frac{\partial \boldsymbol{A}}{\partial t} \mathrm{~d} \tau=\int_{V} \boldsymbol{B} \cdot \nabla \chi \mathrm{d} \tau=\oint_{S} \chi \boldsymbol{B} \cdot \mathrm{d} \boldsymbol{S}=0
$$

and from (7.1),

$$
\int_{V} \boldsymbol{A} \cdot \frac{\partial \boldsymbol{B}}{\partial t} \mathrm{~d} \tau=\int_{V} \boldsymbol{A} \cdot \nabla \times(\boldsymbol{v} \times \boldsymbol{B}) \mathrm{d} \tau=-\oint_{S}(\boldsymbol{A} \cdot \boldsymbol{B}) \boldsymbol{v} \cdot \mathrm{d} \boldsymbol{S}=0,
$$

(recall that $v$ is tangential to the boundary $S$ ).

Hence $\mathrm{d} K / \mathrm{d} t=0$, and to find the relaxed state we need to minimise the magnetic energy $\int_{V} \mathrm{~d} \tau B^{2} / 2 \mu_{0}$ over all variations $\delta \boldsymbol{A}$ subject to the infinity of constraints $K_{\alpha \beta}=$ constant. This leads to the variational problem

$$
\delta \int_{V} \mathrm{~d} \tau\left[\frac{1}{2}(\boldsymbol{\nabla} \times \boldsymbol{A})^{2}-\lambda_{\alpha, \beta} \boldsymbol{A} \cdot \nabla \times \boldsymbol{A}\right]_{\text {all } \delta \boldsymbol{A}, \delta \lambda}=0 .
$$

This is very inconvenient because of the restriction $(\boldsymbol{B} \cdot \nabla \lambda=0)$ on $\lambda$ that is implicit in the use of Clebsch variables $\alpha$ and $\beta$. We can avoid this by introducing the restriction explicitly through another Lagrange multiplier. Then the variational problem becomes

$$
\delta \int_{V} \mathrm{~d} \tau\left[\frac{1}{2}(\boldsymbol{\nabla} \times \boldsymbol{A})^{2}-\lambda_{(x, y, z)} \boldsymbol{A} \cdot \boldsymbol{\nabla} \times \boldsymbol{A}+\mu_{(x, y, z)} \boldsymbol{\nabla} \times \boldsymbol{A} \cdot \nabla \lambda\right]_{\text {all } \delta \boldsymbol{A}, \delta \lambda, \delta \mu}=0 .
$$


We also need to consider the appropriate boundary condition. When the magnetic field is entirely contained within a closed conducting shell, the tangential component of $\delta \boldsymbol{A}$ on the boundary is zero.

Returning to the variational problem, the minimisation requires

$$
\begin{aligned}
\delta W=\delta \boldsymbol{A} \cdot\{\boldsymbol{\nabla} \times(\boldsymbol{\nabla} \times \boldsymbol{A})-2 \lambda \boldsymbol{\nabla} \times \boldsymbol{A}-\boldsymbol{\nabla} \lambda \times(\boldsymbol{A}+\boldsymbol{\nabla} \mu)\} & =0, \\
-\delta \lambda\{(\boldsymbol{\nabla} \times \boldsymbol{A}) \cdot(\boldsymbol{A}+\boldsymbol{\nabla} \mu)\} & =0, \\
+\delta \mu\{(\boldsymbol{\nabla} \times \boldsymbol{A}) \cdot \boldsymbol{\nabla} \lambda\} & =0 .
\end{aligned}
$$

From (7.12), we see that

$$
(\boldsymbol{A}+\nabla \mu)=\boldsymbol{\xi} \times \boldsymbol{B},
$$

therefore

$$
\nabla \lambda \times(\boldsymbol{A}+\nabla \mu) \rightarrow(\boldsymbol{B} \cdot \nabla \lambda) \xi-(\boldsymbol{\xi} \cdot \nabla \lambda) \boldsymbol{B},
$$

where the first term is zero. Then (7.11) becomes

$$
\nabla \times(\nabla \times \boldsymbol{A})-(2 \lambda-\xi \cdot \nabla \lambda) \nabla \times \boldsymbol{A}=0 .
$$

Redefining $\lambda$, we obtain finally

$$
\nabla \times \boldsymbol{B}-\lambda_{\alpha \beta} \boldsymbol{B}=0 .
$$

Now, note well, this cannot be the correct description of the quiescent state, because $\lambda_{\alpha \beta}$ must be determined from the infinity of $K_{\alpha \beta}$, which in turn will reflect every detail of the initial conditions - entirely contrary to the observations.

The resolution of this dilemma is to recognise, as we did earlier, that even small resistivity leads to field line breaking and reconnection. Then it is impossible to identify individual field lines and the individual $K_{\alpha \beta}$ are no longer of any significance. But the sum of all the $K_{\alpha \beta}$ is still invariant. This is just the total magnetic helicity

$$
K_{0}=\int_{V} \boldsymbol{A} \cdot \boldsymbol{B} \mathrm{d} \tau=\text { invariant. }
$$

(Apart from the fact that they are rendered irrelevant by reconnection, the individual $K_{\alpha \beta}$ differ from the total helicity $K_{0}$ in another fundamental way. For the $K_{\alpha \beta}$ to be relevant, we would need to calculate and identify all the field lines - a knowledge of the field $\boldsymbol{B}$ itself is not enough - whereas $K_{0}$ is well defined by the field alone. In a loose analogy with particle dynamics, we could say that $K_{0}$ is an isolating integral, which constrains the available configuration space, whereas the $K_{\alpha \beta}$ are non-isolating integrals, which do not constrain the evolution of the field.)

We conclude, therefore, that the relaxed state of a slightly resistive turbulent plasma is obtained by minimising the magnetic energy subject to a single constraint, here denoted by the Lagrange multiplier $\mu$,

$$
\delta \int\left[\frac{1}{2}(\nabla \times \boldsymbol{A})^{2} \mathrm{~d} \tau-\mu \int \boldsymbol{A} \cdot \boldsymbol{\nabla} \times \boldsymbol{A} \mathrm{d} \tau\right]_{\text {all } \delta \boldsymbol{A}}=0 .
$$

That is

$$
\delta \boldsymbol{A} \cdot\{\boldsymbol{\nabla} \times(\boldsymbol{\nabla} \times \boldsymbol{A})-2 \mu \boldsymbol{\nabla} \times \boldsymbol{A}\}=0,
$$

and upon redefining $\mu$, finally

$$
\boldsymbol{\nabla} \times \boldsymbol{B}=\mu \boldsymbol{B} .
$$

This is no longer any force-free state, depending on the initial conditions; it is a unique state dependent only on a single parameter $\mu$. Thus we have already recovered the uniqueness of the quiescent phase. 


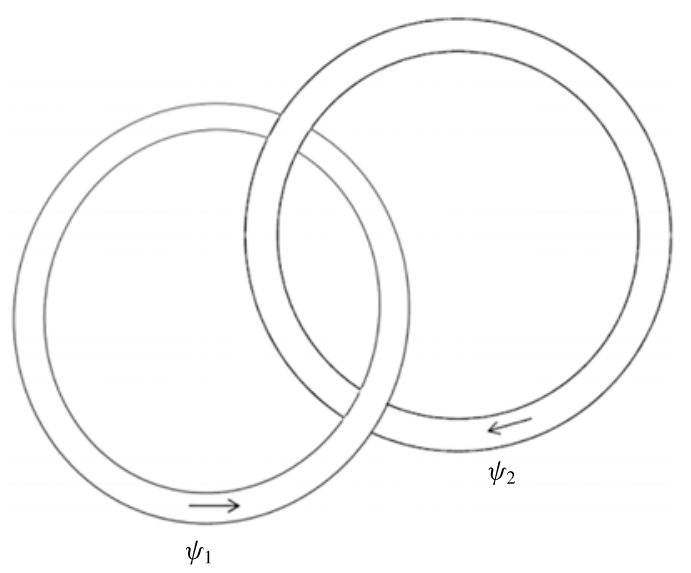

FIGURE 17. Linked flux tubes.

\subsection{Helicity and field line linkage}

Before discussing properties of the relaxed states given by (7.21), we should note some features of the magnetic helicity $K_{0}$.

The helicity $K_{0}=\int \boldsymbol{A} \cdot \boldsymbol{B} \mathrm{d} \tau$ is defined only if the volume of integration is bounded by a closed flux surface. Otherwise it is not gauge-invariant. This reflects the fact that $\boldsymbol{A} \cdot \boldsymbol{B}$ is not a local quantity. One cannot specify where the helicity resides, or a helicity density; only the total helicity within a closed flux surface can be defined.

In fact, helicity is a topological quantity related to the linkage of one flux tube with another. For example, for two flux tubes as in figure 17 , the helicity is $K_{0}=2 \psi_{1} \psi_{2}$ if they are linked as in the figure, and zero if they are not linked (Moffat 1978). Where the helicity is located when they are linked is a meaningless question.

If the volume concerned is multiply connected, as in a torus, then even though the bounding surface is a flux tube, the simple formula (7.18) for $K_{0}$ is not fully gaugeinvariant. The full gauge-invariant form should be

$$
K_{1}=\int_{V} \boldsymbol{A} \cdot \boldsymbol{B} \mathrm{d} \tau-\oint_{C_{1}} \boldsymbol{A} \cdot \mathrm{d} \boldsymbol{l} \oint_{C_{2}} \boldsymbol{A} \cdot \mathrm{d} \boldsymbol{s},
$$

where $C_{1}$ and $C_{2}$ are circuits the long and short way around the torus boundary. However, in most applications the circuit integrals are themselves constant and the simpler form can be used.

\subsection{Back to reality}

If all this sounds too much like exotic mathematics, there is a down-to-earth engineering interpretation of $K_{0}-$ as the volt-seconds from the driving transformer. (Volt-seconds are a standard measure of transformer performance and something you would certainly know was real if you touched the terminals!)

To see this connection, we introduce a small gap in the toroidal shell, as in figure 18 , in order to apply the loop voltage $V_{\text {loop }}$. Then, using (7.22)

$$
\frac{\mathrm{d} K}{\mathrm{~d} t}=\int_{S}\left(\boldsymbol{A} \times \frac{\partial \boldsymbol{A}}{\partial t}\right) \cdot \mathrm{d} \boldsymbol{S}-\oint_{C_{1}} \frac{\partial \boldsymbol{A}}{\partial t} \cdot \mathrm{d} \boldsymbol{l} \oint_{C_{2}} \boldsymbol{A} \cdot \mathrm{d} \boldsymbol{s} .
$$




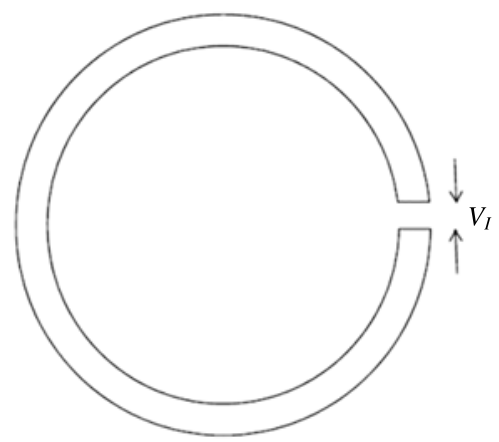

FIGURE 18. Interpreting magnetic helicity in terms of stored volt-seconds.

But $\partial \boldsymbol{A} / \partial t \neq 0$ only in the gap. There we may write $\mathrm{d} \boldsymbol{S}=\mathrm{d} \boldsymbol{l} \times \mathrm{d} \boldsymbol{s}$ and

$$
\int_{S}\left(\boldsymbol{A} \times \frac{\partial \boldsymbol{A}}{\partial t}\right) \cdot \mathrm{d} \boldsymbol{S} \rightarrow-\oint(\boldsymbol{A} \cdot \mathrm{d} \boldsymbol{s}) \oint\left(\frac{\partial \boldsymbol{A}}{\partial t} \cdot \mathrm{d} \boldsymbol{l}\right),
$$

where $\oint(\boldsymbol{A} \cdot \mathrm{d} \boldsymbol{s})=\Psi_{\text {tor }}$ and $\oint\left(\partial_{t} \boldsymbol{A} \cdot \mathrm{d} \boldsymbol{l}\right)=-V_{\text {loop }}$ so

$$
\frac{\mathrm{d} K}{\mathrm{~d} t} \rightarrow 2 \Psi_{\text {tor }} V_{\text {loop }}
$$

Therefore, if loop volts are applied at a constant toroidal flux,

$$
\frac{K}{2 \Psi_{\text {tor }}}=\text { volt-seconds stored in plasma. }
$$

In principle (7.25) and (7.26) describe the start-up of the toroidal plasma, but as this is ill-controlled and volt-seconds are lost in ionisation and heating, we cannot usefully apply them to this initial stage. (The start-up loop voltage is much larger than that required to maintain the relaxed state.)

\subsection{Uniqueness of relaxed states}

In the next few lectures we will discuss specific solutions of equation (7.21) for relaxed states. But first we show that each relaxed state is uniquely determined with no arbitrary or fitted parameters - by the externally controlled quantities $\Psi_{t o r}$ and $K$. To see this, scale all dimensions in the problem by $\mu$. It is then clear, if it was not already, that the solution of (7.21) must be of the form $\boldsymbol{B}=c \hat{\boldsymbol{B}}(\mu \boldsymbol{r} ; \mu a, \mu R)$, $\boldsymbol{A}=(c / \mu) \hat{\boldsymbol{A}}(\mu \boldsymbol{r} ; \mu a, \mu R)$, where $a$ and $R$ are minor and major radii and $c$ is the arbitrary scale factor (Taylor 1974b). Then

$$
K=\int \frac{c^{2}}{\mu} \hat{B}(\rho) \hat{A}(\rho) \frac{\mathrm{d}^{3} \rho}{\mu^{3}} \sim \frac{c^{2}}{\mu^{4}} F(\mu a, \mu R)
$$

and

$$
\Psi_{t o r}=\int c \hat{B}(\rho) \frac{\mathrm{d}^{2} \rho}{\mu^{2}} \sim \frac{c}{\mu^{2}} G(\mu a, \mu R),
$$

giving

$$
\frac{K}{\Psi_{t o r}^{2}}=\text { function of }(\mu a, \mu R)
$$

which fixes $\mu$. The scale factor $c$ can then be set from $K$ or $\Psi_{t o r}$. 


\subsection{Summary}

Now let us summarise what we have seen so far. Observations of turbulent plasmas in a toroidal pinch, with initial toroidal field $B_{0}$ and current $I$, have shown that quiescent states form that depend only on the parameter $\theta \equiv 2 I / a B_{0}$, and if $\theta>\theta_{c}$ the toroidal field spontaneously reverses.

We interpret these as 'relaxed' states, that is, states of minimum energy subject to constraints. In an ideal plasma there would be an infinity of constraints, but this leads to a state in strong disagreement with observations. In a slightly resistive plasma, the infinity of individual constraints become irrelevant and the only surviving constraint is that the total magnetic helicity $K_{\alpha \beta}=\int_{\alpha \beta} \boldsymbol{A} \cdot \boldsymbol{B} \mathrm{d} \tau$ is invariant. This leads to relaxed states in agreement with observations, as we shall see in detail in the following sections.

\section{Relaxed states in toroidal pinch}

Now, at last, we turn to calculating a relaxed state. We first consider a toroidal pinch surrounded by a conducting shell, represented by the boundary condition $\boldsymbol{B} \cdot \boldsymbol{n}=0$ (see figure 4). As we know, such a system has two invariants, $K$ and $\psi_{t o r}$, and these completely specify the relaxed state.

For simplicity, we first solve $\boldsymbol{\nabla} \times \boldsymbol{B}=\mu \boldsymbol{B}$ for the relaxed state in a cylinder, which can be considered an approximation for a large-aspect-ratio toroidal pinch. The magnetic field then depends only on the minor radius, so that $B_{r}=0, B_{\theta}=B_{\theta}(r)$, $B_{z}=B_{z}(r)$ and

$$
\begin{gathered}
-\frac{\partial B_{z}}{\partial r}=\mu B_{\theta}, \\
\frac{1}{r} \frac{\partial}{\partial r}\left(r B_{\theta}\right)=\mu B_{z},
\end{gathered}
$$

so

$$
\frac{1}{r} \frac{\partial}{\partial r}\left(r \frac{\partial B_{z}}{\partial r}\right)+\mu^{2} B_{z}=0
$$

Therefore

$$
B_{z}=\alpha \mathrm{J}_{0}(\mu r), \quad B_{\theta}=\alpha \mathrm{J}_{1}(\mu r) .
$$

Unsurprisingly, this is known as the Bessel function profile. Integration of (8.2) shows that the pinch ratio $\theta=\mu a / 2$. A further calculation relates the parameter $\mu$ to $K / \Psi_{\text {tor }}^{2}$ as in (7.29),

$$
\frac{K}{\Psi^{2}}=\frac{l}{2 \pi a}\left\{\frac{\mu a\left[\mathrm{~J}_{0}^{2}(\mu a)+\mathrm{J}_{1}^{2}(\mu a)\right]-2 \mathrm{~J}_{0}(\mu a) \mathrm{J}_{1}(\mu a)}{\mathrm{J}_{1}^{2}(\mu a)}\right\}
$$

where $l$ is the cylinder length, identified with $2 \pi R$ in a torus.

The Bessel profile fits the experimental data remarkably well, as can be seen in figure 19 and in Bodin (1983, 1984, 1987).

\subsection{Spontaneous field reversal}

Since the 'toroidal' field is $B_{z} \sim \mathrm{J}_{0}(\mu r)$, it is clear that the onset of spontaneous field reversal in the relaxed state occurs at $\mu a>2.4$. This corresponds to an experimental pinch parameter $\theta>1.2$ - which again agrees well with the experimental observations. 


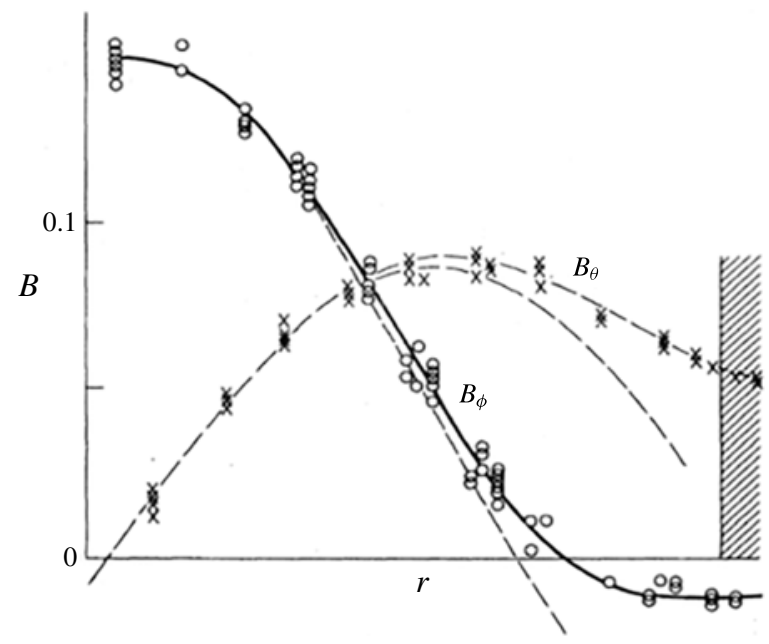

FIgURE 19. Comparison of experimental magnetic field profiles in the quiescent state of the toroidal pinch HBTX-1A to the theoretical expressions (8.4). (Reproduced with permission from Bodin (1984).)

The experimental data from reverse field pinches (RFPs) is usually presented in the form of an $F-\theta$ diagram, where

$$
F \equiv \frac{B_{\text {toroidal }}^{(\text {wall })}}{\left\langle B_{\text {toroidal }}\right\rangle},
$$

and the angled brackets denote the average over the cross-section. The value of $F$ in the relaxed state is

$$
F=\frac{2 \theta \mathbf{J}_{0}(2 \theta)}{\mathbf{J}_{1}(2 \theta)} .
$$

Note that this is calculated from 'first principles', and contains no empirical or fitted factors.

Equation (8.7) is in good agreement with experiment, as can be seen in figure 20 . Note that the experimental points from several machines lie on a universal curve, close to the theoretical one but at somewhat higher $\theta$. (This may be due to the lower current density near the wall, where the plasma is contaminated by impurities.)

Even more striking evidence for relaxation can be seen in the time-dependent behaviour shown in figure 21. We see that if the operating point $(F, \theta)$ is forced away from the locus of relaxed states (e.g. by a temporary increase in loop voltage), it very quickly $(0.8 \mu \mathrm{s}$ ! - comparable to the Alfvén time $\sim 1 \mu \mathrm{s})$ reverts back to it. However, as long as the operating point is changed slowly, the plasma stays close to the theoretical relaxed-state curve (figures $21 b$ and 22).

\section{Energetics of toroidal relaxed states}

Actually, determination of the relaxed state is more complex than the analysis so far suggests. This is because there may be more than one solution of (7.21) compatible with $K, \Psi$ and the boundary conditions. Therefore, in any situation we must consider all possible solutions of (7.21) and select that which has the lowest energy. 


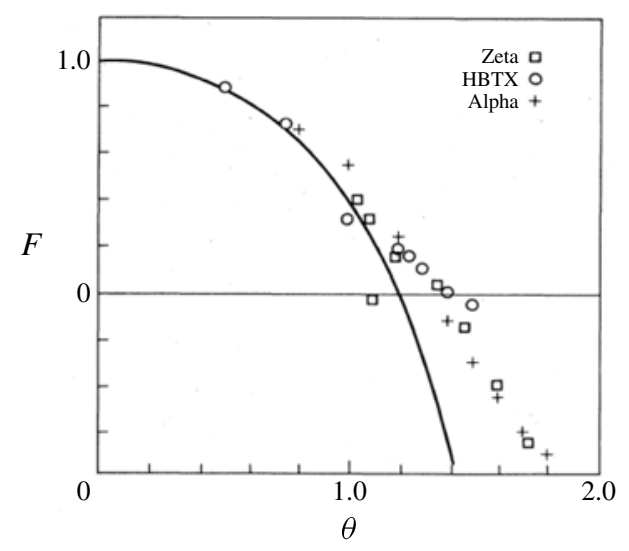

FIgURE 20. An $F-\theta$ diagram showing data from the pinch experiments HBTX1, ALPHA and ZETA and the theoretical curve. (Reproduced with permission from Bodin \& Newton (1980).)

(a)

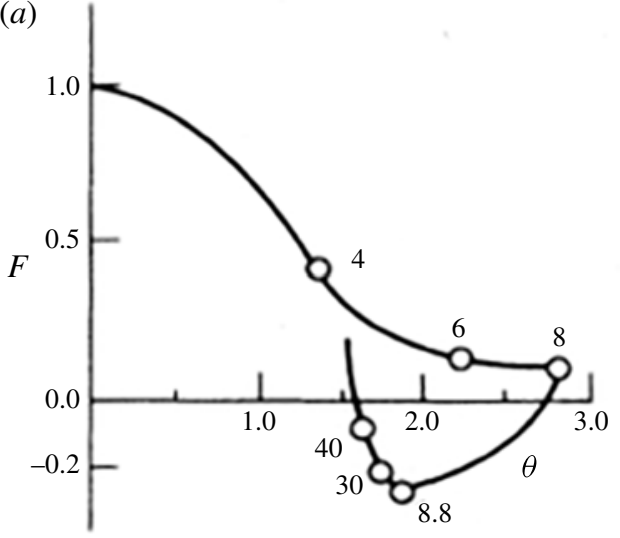

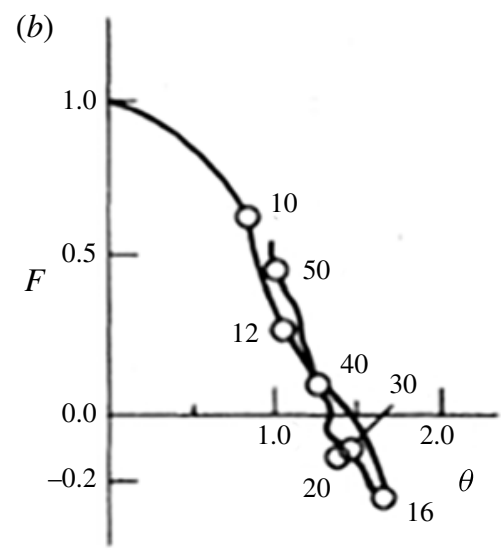

FIgURE 21. Time-dependent $F-\theta$ curve for HBTX1 in $(a)$ fast mode and $(b)$ slow mode. Time intervals are given in microseconds. (Reproduced with permission from Bodin \& Newton (1980).)

In a cylinder we can find all the solutions of (7.21) explicitly (Taylor 1968). Any solution, whether axisymmetric or not, satisfies

$$
\begin{gathered}
\frac{1}{r} \frac{\partial}{\partial \theta} B_{z}-\frac{\partial}{\partial z} B_{\phi}=\mu B_{r}, \\
\frac{\partial B_{r}}{\partial z}-\frac{\partial B_{z}}{\partial r}=\mu B_{\theta}, \\
\frac{1}{r} \frac{\partial}{\partial r}\left(r B_{\theta}\right)-\frac{1}{r} \frac{\partial B_{r}}{\partial \theta}=\mu B_{z}, \\
\nabla \times(\nabla \times B)=\mu^{2} \boldsymbol{B} .
\end{gathered}
$$



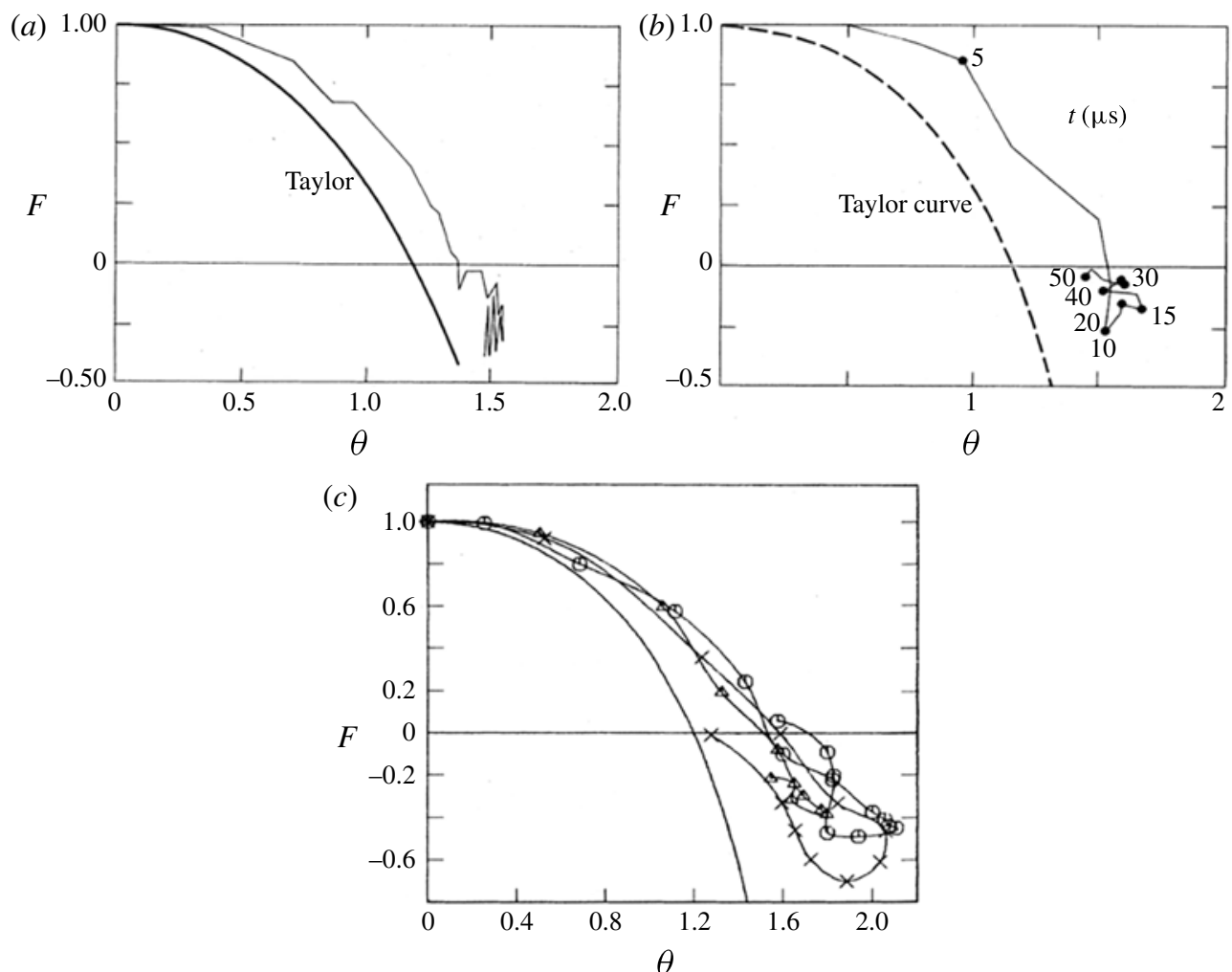

FIgURE 22. Time-dependent $F-\theta$ curves from pinches operated in slow mode: (a) ZT40 experiment (from DiMarco 1983), (b) TPRE experiment (reproduced with permission from Tamaru et al. 1979) and (c) REPUTE experiment (reproduced with permission from Toyama et al. 1985).

Therefore, the most convenient starting point is to first find $B_{z}$ from

$$
\nabla^{2} B_{z}+\mu^{2} B_{z}=0
$$

and then compute the other components of $\boldsymbol{B}$ from (9.4).

The general solution of (9.5) can be expressed as a sum of Fourier modes $B_{z}=$ $h(r) \cos (m \theta+k z)$, where

$$
\frac{1}{r} \frac{\partial}{\partial r}\left(r \frac{\partial h}{\partial r}\right)-\left(\frac{m^{2}}{r^{2}}+k^{2}\right) h+\mu^{2} h=0,
$$

so that

$$
h(r)=\mathbf{J}_{m}(y), \quad \text { where } y \equiv r\left(\mu^{2}-k^{2}\right)^{1 / 2} .
$$

The general form of the relaxed state in a cylinder therefore becomes

$$
\boldsymbol{B}=\sum_{m, k} a_{m k} \boldsymbol{B}^{m k}(\boldsymbol{r})
$$

where

$$
B_{r}^{m k}=-\frac{1}{\left(\mu^{2}-k^{2}\right)^{1 / 2}}\left\{k \mathbf{J}_{m}^{\prime}(y)+\frac{m \mu}{y} \mathbf{J}_{m}(y)\right\} \sin (m \theta+k z),
$$




$$
\begin{gathered}
B_{\theta}^{m k}=-\frac{1}{\left(\mu^{2}-k^{2}\right)^{1 / 2}}\left\{\mu \mathbf{J}_{m}^{\prime}(y)+\frac{m k}{y} \mathbf{J}_{m}(y)\right\} \cos (m \theta+k z), \\
B_{z}^{m k}=\mathbf{J}_{m}(y) \cos (m \theta+k z) .
\end{gathered}
$$

Note that this is an exact solution.

In the general solution $(9.8)$, the $(m=0, k=0)$ term is of a different character to all the others. It satisfies the boundary condition $B_{r}=0$ for any value of $\mu$ and it carries non-zero toroidal flux. All other terms satisfy the boundary condition only for discrete values of $\mu\left(=\mu_{m k}\right)$ given by

$$
k \mathrm{~J}_{m}^{\prime}\left[\left(\mu^{2}-k^{2}\right)^{1 / 2} a\right]+\frac{m \mu}{\left(\mu^{2}-k^{2}\right)^{1 / 2} a} \mathbf{J}_{m}\left[\left(\mu^{2}-k^{2}\right)^{1 / 2} a\right]=0,
$$

and they carry no toroidal flux. Consequently, there are two distinct types of relaxed state in a cylinder.

Type I: the primitive or symmetric state, consisting solely of the $(m=0, k=0)$ term. This is the Bessel state we have already discussed, in which $\mu$ is determined by $K / \Psi^{2}$.

Type II: a mixed or helical state made up of an $(m=0, k=0)$ term, to provide the required toroidal flux, plus one term with $m$ and $k$ non-zero, i.e. $\boldsymbol{B}=\alpha_{0} \boldsymbol{B}^{00}+$ $\alpha_{m k} \boldsymbol{B}^{m k}$ (no summation over $m, k$ ). In this mixed state $\mu(m, k)$ is given by (9.12). Consequently, $\mu$ is no longer determined by $K / \Psi^{2}$; instead, with $\mu$ fixed, $K / \Psi^{2}$ determines the mixing ratio $\alpha_{m k} / \alpha_{0}$.

Both Type I and Type II relaxed states are therefore fully determined by $K$ and $\Psi$, but in quite different ways. We now need to find which relaxed state has the lowest energy, and, if it is a Type II, what are the specific values of $m$ and $k$.

\subsection{Primitive and mixed states}

The task of finding which is the lowest-energy solution is made easier by two comparison theorems (Taylor 1968):

(i) The first theorem is that if two solutions have the same value of $\Psi$ and $K$, then the one with the smaller $\mu$ has the lower energy. The proof of this is as follows.

If $\int \boldsymbol{A}_{1} \cdot \boldsymbol{B}_{1} \mathrm{~d} \tau=\int \boldsymbol{A}_{2} \cdot \boldsymbol{B}_{2} \mathrm{~d} \tau$ with $\boldsymbol{A}_{1}=\boldsymbol{A}_{2}$ on the boundary, and both $\boldsymbol{\nabla} \times \boldsymbol{B}_{1}=\mu_{1} \boldsymbol{B}_{1}$ and $\boldsymbol{\nabla} \times \boldsymbol{B}_{2}=\mu_{2} \boldsymbol{B}_{2}$, then

$$
\int\left[\nabla \times\left(\boldsymbol{A}_{1}-\boldsymbol{A}_{2}\right)\right]^{2} \mathrm{~d} \tau=\left(\mu_{2}+\mu_{1}\right) \int\left(\boldsymbol{A}_{2}-\boldsymbol{A}_{1}\right) \cdot \nabla \times \boldsymbol{A}_{2} \mathrm{~d} \tau
$$

and

$$
\int\left(\boldsymbol{\nabla} \times \boldsymbol{A}_{2}\right)^{2} \mathrm{~d} \tau-\int\left(\boldsymbol{\nabla} \times \boldsymbol{A}_{1}\right)^{2} \mathrm{~d} \tau=\left(\mu_{2}-\mu_{1}\right) \int\left(\boldsymbol{A}_{2}-\boldsymbol{A}_{1}\right) \cdot \nabla \times \boldsymbol{A}_{2} \mathrm{~d} \tau .
$$

Therefore, if $W_{1}$ and $W_{2}$ are the energies of two relaxed states, then

$$
W_{2}-W_{1}=\frac{\mu_{2}^{2}-\mu_{1}^{2}}{\left(\mu_{2}+\mu_{1}\right)^{2}} \int \frac{\left(B_{2}-B_{1}\right)^{2}}{2} \mathrm{~d} \tau .
$$

Hence, if $\mu_{2}^{2}>\mu_{1}^{2}, W_{2}>W_{1}$.

(ii) The second theorem is that the eigenvalues of (9.12) for the same $k$ increase with increasing $m$. The proof of this is similar to the argument above and to the Sturm-Liouville comparison theorems in the theory of differential equations (Ince 1956).

By theorem (i), to find the solution with the lowest energy, we must select that with the smallest $\mu$. For a Type I solution this means taking the smallest root of (8.5). 


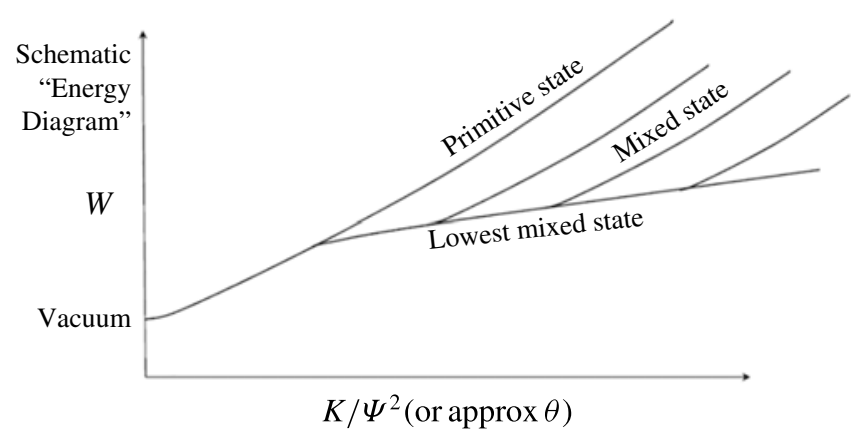

FIGURE 23. Illustration of the energy of the relaxed states in a cylindrical plasma as a function of $K / \Psi^{2}$.

By theorem (ii), for a Type II solution it means taking $m=1$ and then searching through $k$ at $m=1$ to find the smallest eigenvalue of (9.12). This occurs at $k a \approx 1.25$ and has the value $\mu a=3.11$.

This essentially completes the solution of our problem. The primitive solution $\boldsymbol{B}=$ $\alpha \boldsymbol{B}^{00}$ is the correct relaxed state so long as $\mu a<3.11$, i.e. $\theta \leqslant 1.6$. Otherwise, the correct state is a mixed, helical state, $\boldsymbol{B}=\alpha_{0} \boldsymbol{B}^{00}+\alpha_{m k} \boldsymbol{B}^{m k}$, with $m=1, k=1.25$ and $\mu a=3.11$.

Recalling that in the primitive state $\mu a=F\left(K / \Psi^{2}\right)$, this is equivalent to saying that the primitive state is the correct lowest-energy state for $K / \Psi^{2}$ less than some critical value, and the mixed state is the correct one for $K / \Psi^{2}$ greater than this value. This situation is illustrated schematically in figure 23. Equally, of course, we could say that the primitive state occurs when the volt-seconds are below a critical value and the mixed state occurs when the volt-seconds exceed that value.

\subsection{Current saturation and spontaneous symmetry breaking}

The Type II helical states with $\mu a=3.11(\theta \sim 1.6)$ have a remarkable property. In these states, since $\mu$ is fixed, an increase in volt-seconds (at fixed $\Psi$ ) produces no increase in the toroidal current $I$ ! In other words, a helical relaxed state acts as a current-limiting circuit element! When the voltage is increased, instead of producing an increase in current, the current remains the same but flows in a tighter helix with higher inductance. (This is reminiscent of the flexible wire with which we began the discussion of relaxed states!)

Evidence for this second type of relaxed state, and for current saturation, is shown in figure 24. This shows discharges in which $\theta$ is initially driven to a large value. However, it then falls quickly to $\sim 1.6$ and remains there for the rest of the discharge. (Some discharges can be prevented from relaxing and held at $\theta>1.6$, but at the cost of increased fluctuation levels and power losses; see figure 25.)

The transition from a primitive axisymmetric state to a helical state means that relaxation theory predicts not one, but two critical values of the pinch parameter $\theta$ :

(i) at $\theta \approx 1.2 \rightarrow$ spontaneous field reversal occurs, but the plasma remains axisymmetric (see $\S 8.1$ );

(ii) at $\theta \approx 1.6 \rightarrow$ current saturation occurs and a helical deformation sets in (see $\S 9.1)$.

Note that the second transition, from an axisymmetric state to the helical mixed state, is a neat example of 'spontaneous symmetry breaking'. 

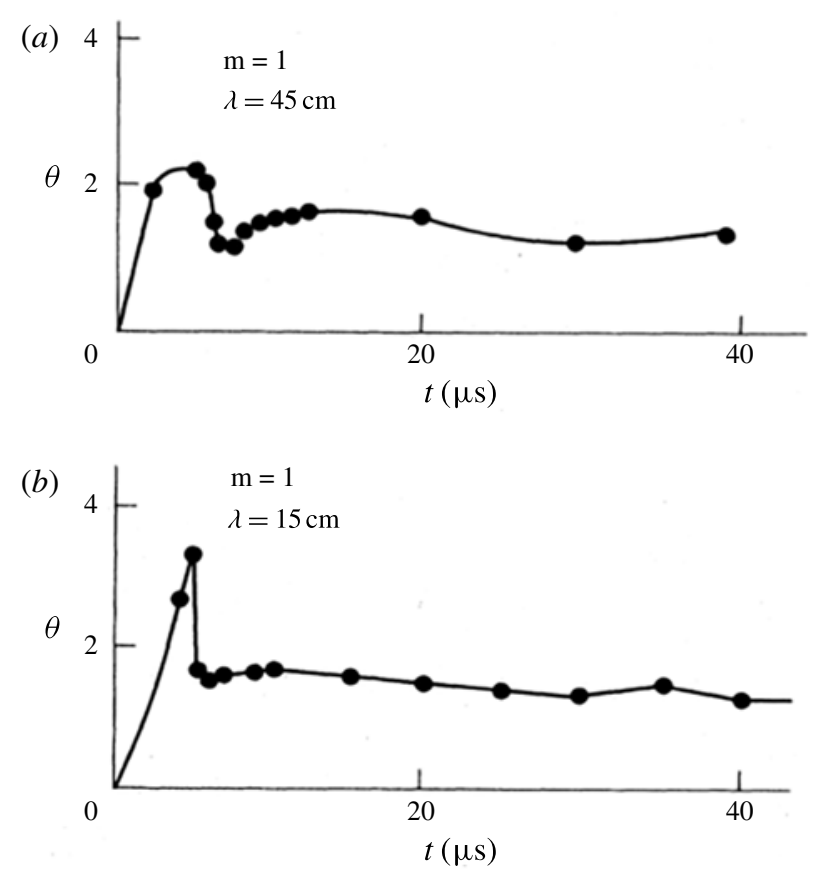

FIGURE 24. Evidence for second critical $\theta$ in HBTX1. (Reproduced with permission from Bodin \& Newton (1980).)

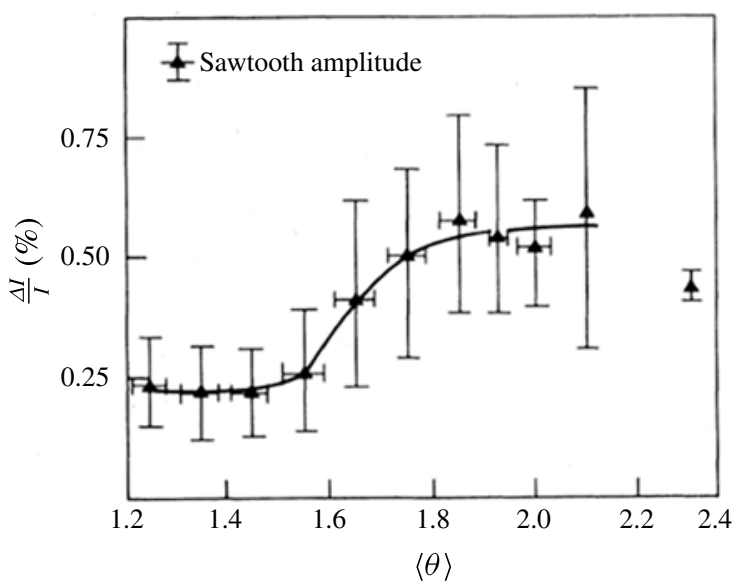

FIGURE 25. Driving a toroidal pinch current above the critical value. Fluctuation level versus $\theta$ in ZT-40. (Reproduced with permission from Watt \& Nebel (1983). Copyright 1983, AIP Publishing LLC.)

\subsection{Stability}

Like all relaxed states, the relaxed states of the toroidal pinch are 'minimum energy' and therefore stable against any perturbations that do not change the helicity. This, of course, includes all ideal MHD perturbations. 


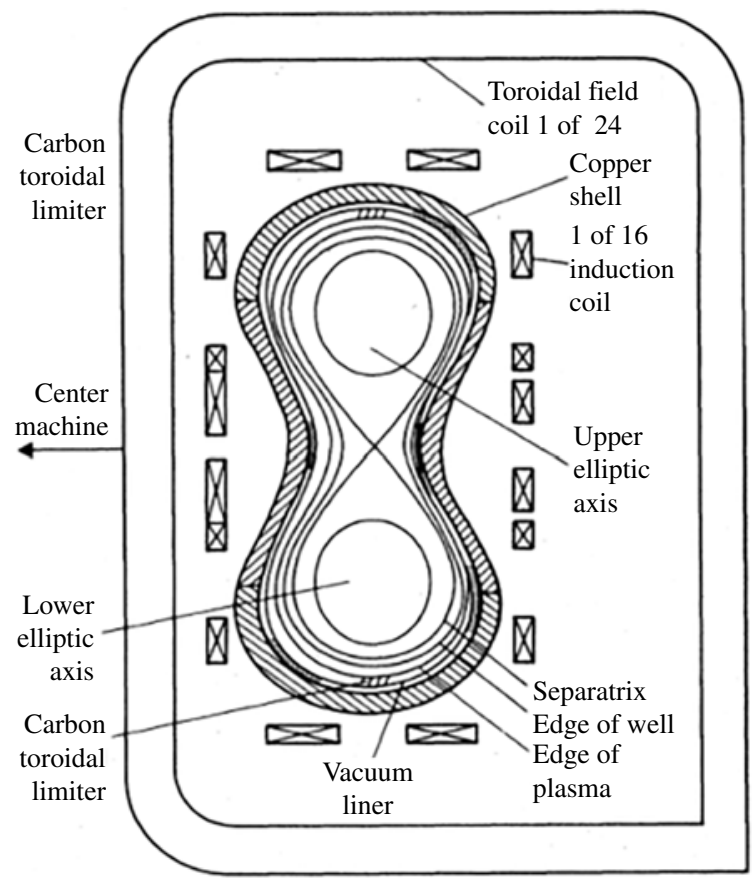

FIGURE 26. Multipinch experiment at General Atomics. (Reproduced with permission from La Haye et al. (1986).)

The transition from Type I to Type II (helical) relaxed states at $\mu a=3.11$ coincides with a linear stability boundary for resistive tearing modes. However, the present analysis is not restricted to the linear regime. The nonlinear amplitude of the helical deformation is given precisely in terms of $K / \Psi^{2}$ as (Martin \& Taylor 1974; Taylor 1986)

$$
\left(\frac{\alpha_{1}}{\alpha_{0}}\right)=0.47\left(\frac{2 \pi a}{l} \frac{K}{\Psi^{2}}-8.21\right)^{1 / 2} .
$$

\section{The multipinch}

The 'multipinch', an experiment carried out at General Atomics (La Haye et al. 1986), is important because it shows that relaxation occurs in experiments other than the RFP, and because its relaxed states show some striking and unexpected features. The cross-section of the multipinch resembles a figure eight with height $(50 \mathrm{~cm})$ and width $(20 \mathrm{~cm})$ chosen to resemble two circular toroidal pinches one above the other, connected at the 'waist' of the figure eight (see figure 26). The major radius of the torus is $\sim 52 \mathrm{~cm}$ and the start-up operation is similar to that of orthodox RFPs.

It is not difficult to calculate the axisymmetric relaxed states of the multipinch (La Haye et al. 1986; Taylor 1986). (Note that we are now discussing a finite-aspectratio torus with non-circular cross-section, not an infinite cylinder.) Using cylindrical coordinates $(R, \phi, z)$, the magnetic field can be written as

$$
\boldsymbol{B}=\frac{\hat{e}_{\phi} \times \nabla \chi}{R}+\hat{e}_{\phi} \frac{f}{R} .
$$


(a)

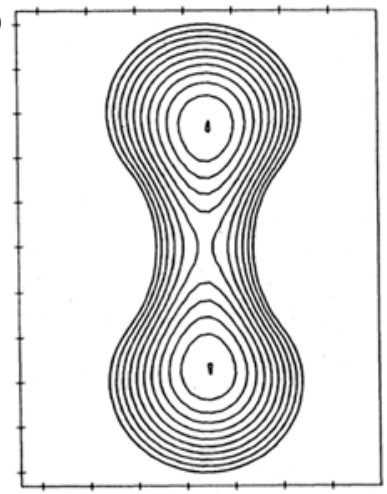

(b)

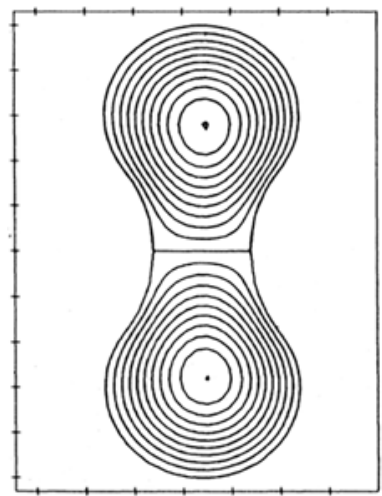

FIGURE 27. Relaxed profiles in the multipinch: (a) primitive relaxed state, with $\mu a=1.5$ and $(b)$ lowest eigenfunction, with $\mu a=2.21$. (Reproduced with permission from La Haye et al. (1986).)

Then, in the relaxed state $\nabla \times \boldsymbol{B}=\mu \boldsymbol{B}$, we have $\nabla f=-\mu \nabla \chi$, so that $f=C-\mu \chi$, and

$$
\Delta^{+} \chi \equiv \frac{\partial^{2} \chi}{\partial z^{2}}+R \frac{\partial}{\partial R}\left(\frac{1}{R} \frac{\partial \chi}{\partial R}\right)=\mu f .
$$

The boundary condition is $\chi=$ constant, which without loss of generality can be set to zero. The constant $C$ is fixed by the toroidal flux $\Psi$,

$$
C=\mu\langle\chi\rangle+\langle R\rangle \Psi / A
$$

where $A$ is the cross-sectional area and the averages are defined as

$$
\langle f\rangle \equiv\left(\int \frac{f}{R} \mathrm{~d} R \mathrm{~d} z\right)\left(\int \frac{1}{R} \mathrm{~d} R \mathrm{~d} z\right)^{-1} .
$$

Then axisymmetric relaxed states are given by

$$
\Delta^{+} \chi+\mu^{2}(\chi-\langle\chi\rangle)=\mu\langle R\rangle \Psi / A .
$$

After our experience with the RFP we can anticipate that the eigenfunctions corresponding to $(10.5)$,

$$
\Delta^{+} \chi+\mu^{2}(\chi-\langle\chi\rangle)=0
$$

will also be needed to interpret the behaviour of the multipinch.

Equations (10.5) and (10.6) are Grad-Shafranov-like equations, albeit unorthodox in that they are non-local through $\langle\chi\rangle$. A solution of (10.5) for a relaxed state is shown in figure $27(a)$. In addition to being axisymmetric, it is also symmetric about the equatorial mid-plane, with $B_{\phi}$ the same sign in the upper and lower lobes. The lowest axisymmetric eigenfunction, calculated from (10.6), has eigenvalue $\mu a=2.21$, and is shown in figure $27(b)$. Note that this is antisymmetric about the equatorial plane, with $B_{\phi}$ having opposite sign in the upper and lower lobes. It therefore carries no net toroidal flux. 

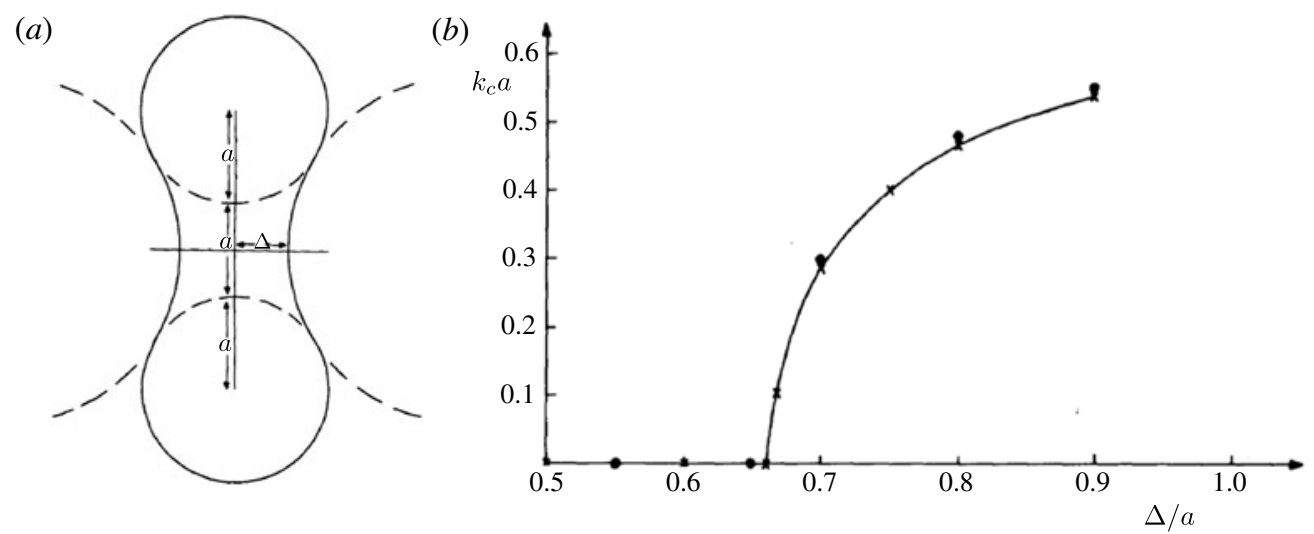

FIGURE 28. (a) Idealised multipinch and $(b)$ toroidal wavenumber corresponding to the lowest eigenvalue as a function of $\Delta$. (Reproduced with permission from Gimblett et al. (1987). Copyright 1987, AIP Publishing LLC.)

The toroidal field $f$ in the relaxed state is given by

$$
\Delta^{+} f+\mu^{2} f=0
$$

with $f$ constant on the boundary. The value of this constant is again fixed by the toroidal flux

$$
\langle f\rangle=\frac{\langle R\rangle}{A} \Psi .
$$

The lowest axisymmetric eigenvalue can also be calculated from (10.7) by imposing the restriction $\langle f\rangle=0$, i.e. zero toroidal flux. But do not confuse this zero-flux eigenvalue with that defined by the boundary condition $f=0$. The latter determines a field reversal point analogous to $\theta=1.2$ in RFPs. In the multipinch, the zero-flux and field reversal eigenvalues are close together, $\mu a=2.21$ and $\mu a=2.18$, respectively (see discussion below).

\subsection{Nature of the eigenfunctions in multipinch}

In the same way that we had to determine which of many eigenfunctions in a cylinder had the lowest energy, we still have to decide whether the lowest eigenvalue in the multipinch is in fact associated with an axisymmetric eigenfunction. In a cylinder the lowest eigenfunction was helical, but in a re-entrant cross-section the reverse may be the case.

To see why, consider the extreme case of a multipinch made up of two circular tori, one above the other, connected only by a narrow slit where they make contact. In this case an axisymmetric eigenfunction, carrying no net flux, could be constructed from a circular cylinder solution in each torus, one carrying positive toroidal flux and the other carrying equal negative flux. To be continuous, the toroidal field would then have to vanish at the slit - so the two cylinder solutions must have $\mu a \sim 2.4$, much lower than the helical eigenvalue $\mu a \sim 3.11$. This suggests that the lowest eigenfunction in a multipinch is axisymmetric, at least when the 'waist' is narrow. (It also explains why the field reversal point is so close to the zero-flux eigenvalue.)

Numerical computations (Gimblett et al. 1987) confirm this picture. Figure 28 shows the toroidal wavenumber of the lowest eigenfunction for an idealised linear 

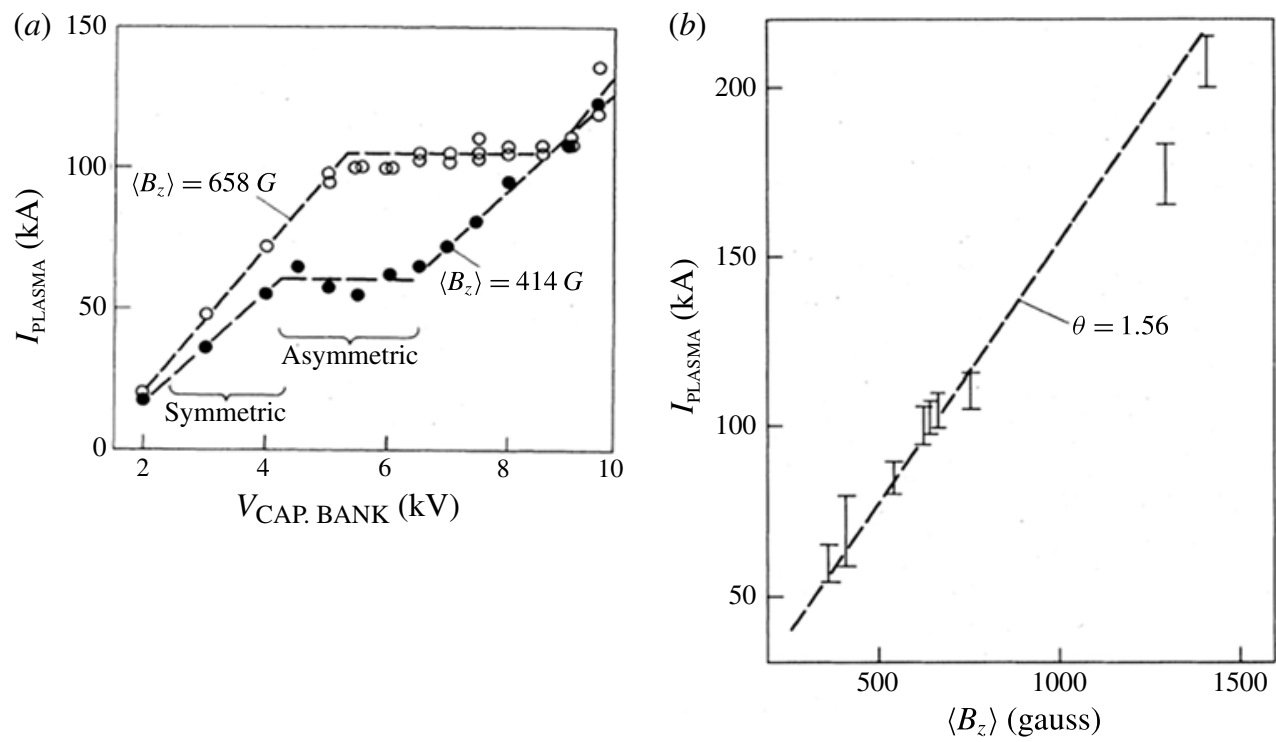

FIgURE 29. (a) Plasma current versus driving voltage and (b) variation of saturation current with toroidal flux in the multipinch. The value $\theta=1.56$ corresponds to $\mu a=2.42$ here. (Reproduced with permission from La Haye et al. (1986).)

multipinch, with cross-section made up of circular arcs. When the 'waist' $\Delta$ is comparable with the diameter of the lobes $(\Delta / a \sim 1$ so there is essentially no indentation at the waist), the helical wavelength $2 \pi / k_{c}$ is $\sim 10$ times the lobe width. As the waist is narrowed, the wavelength increases and becomes infinite, that is, the eigenfunction becomes axisymmetric, when $\Delta=0.66$. (This is somewhat larger than the actual experiment, but its cross-section is not made up of circular arcs.)

We can now describe the properties we would expect of relaxed states in the multipinch.

(i) For small $K / \Psi^{2}$ (low volt-seconds), the relaxed state will be axisymmetric and symmetric about the equator/mid-plane, as in figure $27(a)$. In this state, plasma current is the same in both lobes and increases with increasing volt-seconds.

(ii) When $K / \Psi^{2}$, or the volt-seconds, reach a critical value, the relaxed state becomes a superposition of the axisymmetric state and the eigenfunction shown in figure $27(b)$. Therefore it continues to be axisymmetric but is no longer symmetric about the mid-plane. As the volt-seconds (at fixed toroidal flux) increase further, more current flows in one lobe and less in the other, but the total current does not change. Also, this saturated total current is proportional to the toroidal flux $\Psi$.

\subsection{Experimental results}

The features described above are clearly demonstrated in the experiment. Figure 29 shows the peak plasma current as a function of the driving transformer voltage $V_{C B}$, at fixed $\Psi$. (Plasma current is a measure of $\mu a$ and $V_{C B}$ is a rough measure of $K / \Psi^{2}$.) Note the following:

(i) At low $V_{C B}$, the current is axisymmetric and symmetric about the equatorial midplane, i.e. equal in the upper and lower lobes, and it increases with $V_{C B}$. 
(ii) Above a critical $V_{C B}$, the current remains axisymmetric but ceases to be symmetric about the mid-plane and it no longer increases with $V_{C B}$. The total current saturates but more of it flows in one lobe and less in the other, and this asymmetry increases as $V_{C B}$ increases.

(iii) The saturation current, $I_{s a t}$, is proportional to the toroidal flux - in agreement with the relaxed-state prediction, $I_{s a t} / \Psi \propto \mu a$ (see figure $29 b$ ).

(iv) The observed proportionality between $I_{\text {sat }}$ and $\Psi$ corresponds to $\mu a \sim 2.42$, in reasonable agreement with the theoretical value $\mu a=2.21$ for the zero-flux eigenvalue.

\subsection{Flux generation}

In fact, all the features of the experiment agree well with the properties calculated for the relaxed states - except one! According to theory, the total current should remain at its saturated value indefinitely as $V_{C B}$ increases beyond the critical value corresponding to $\mu=2.21$. But this is not what happens in the experiment: at some point the current starts to increase again. The reason for this is that, as $V_{C B}$ increases, less and less current flows in one lobe and more in the other. This imbalance increases until at some point essentially all the current flows in one lobe and very little in the other. If current saturation were to continue beyond this point, as in theory it should, the current would have to reverse in one lobe. Instead, the discharge dies out in that lobe and becomes confined to the other, where it acts as a simple toroidal pinch whose current increases with $V_{C B}$.

This feature of the multipinch brings out an important aspect of relaxation: it is not a passive decay process. It involves the generation of flux and current by plasma turbulence - as in a turbulent dynamo. When the multipinch reaches the state in which essentially all the current is in one lobe, and any further advance would require reverse current, the 'dynamo' is unable to act through the narrow gap from one lobe to the other - so the current remains confined to one lobe.

Finally we should note that current saturation in the multipinch is another example of spontaneous symmetry breaking, as it was in the cylinder. However, the symmetry involved is different in the two systems. In the cylinder it is the axial symmetry that is broken, whereas in the multipinch it is the symmetry about the equatorial plane.

\section{Spherical systems I: spheromak}

Relaxation also occurs in spherical systems, that is, systems that are not multiply connected as is a torus. This topological change has a profound effect on the nature of the relaxed states (Turner et al. 1983; Taylor 1985, 1986, 1992).

The simplest spherical system is the spheromak, shown in figure 30 . This has nested toroidal flux surfaces that may appear similar to those in a toroidal pinch. The crucial difference is that in the spheromak there is no central aperture (no hole in the doughnut) to permit toroidal field coils. This implies the following:

(i) Reconnection can occur across the axis so that toroidal flux need not be conserved.

(ii) The toroidal field $B_{\phi}$ vanishes everywhere on the boundary. But this does not imply $q=0$, as it would in a toroidal system. In fact, in a spheromak $q$ is remarkably uniform in the relaxed state, with $q=0.825$ on the magnetic axis and $q=0.72$ at the wall. The finite value of $q$ at the wall arises because $q(\psi)$ is an average over a flux surface and near the wall this involves taking a limit in 


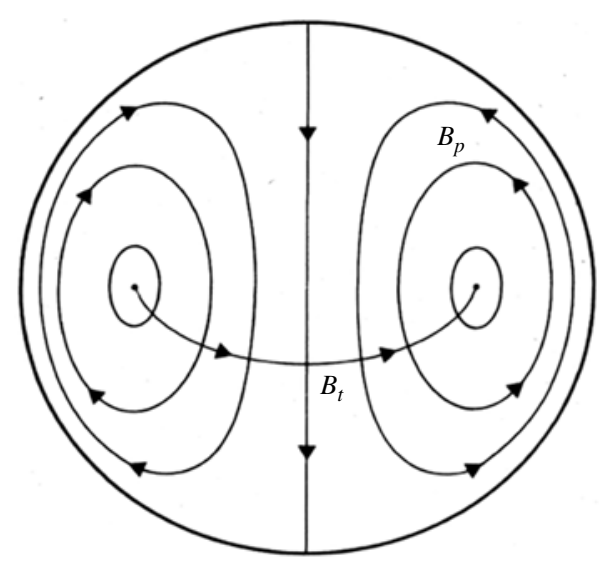

FIGURE 30. Schematic of a spheromak configuration (from Taylor 1986).

which both $B_{\text {toroidal }}$ and $B_{\text {poloidal }}$ tend to zero at the poles, with the ratio leading to a finite $q(\Psi)$.

As there can be no transformer core through a spheromak, we cannot use this to create a plasma. Instead, there are three main techniques for creating a spheromak:

(i) coaxial gun injection,

(ii) combined $\theta-z$ discharges and

(iii) an inductive flux core.

These are illustrated in figure 31, and the details of their operation can be found in Taylor (1986) and references therein.

\subsection{Relaxed states of a spheromak}

The helicity $K=\int \boldsymbol{A} \cdot \boldsymbol{B} \mathrm{d} \tau$ is conserved in a spheromak during relaxation (see $\S 7.1$ ), and the relaxed state satisfies

$$
\nabla \times(\nabla \times A)=\mu \nabla \times A .
$$

Now, we recall that in a torus there were two invariants, $K$ and $\Psi$, which were just sufficient to determine the relaxed state. However, in a spheromak, we have only one invariant, the helicity $K$. The toroidal flux is no longer invariant because annihilation of flux can occur across the axis of symmetry. How, then, can the relaxed state be unique in a spheromak?

The key to this question is that in a torus there were two independent loop integrals on the surface of the torus: $\oint \boldsymbol{A} \cdot \mathrm{d} \boldsymbol{l}$, the flux through the 'hole' in the torus; and $\oint \boldsymbol{A} \cdot \mathrm{d} \boldsymbol{s}=\Psi$, the toroidal flux inside the torus. But in a spheromak, being simply connected, all loop integrals on the surface are identical. In fact, since there is no flux through the boundary, they are all zero. Apart from an irrelevant gauge transformation this is equivalent to $\boldsymbol{A} \equiv 0$ on the boundary of a spheromak. But this is just the spheromak eigenvalue condition!

Hence for a spheromak, the only relaxed state is the lowest eigenfunction: the eigenvalue determines $\mu$ and the field amplitude is determined by the sole invariant $K$. This means that, apart from a scale factor, the relaxed state in a spheromak is unique and entirely determined by the shape of the container. 
(a)
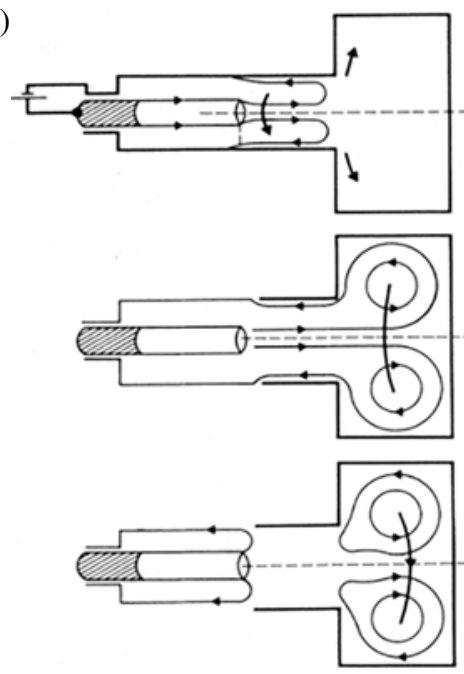

(b)
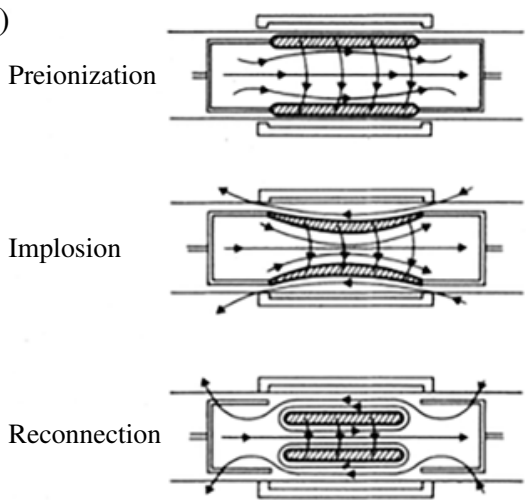

Equilibrium

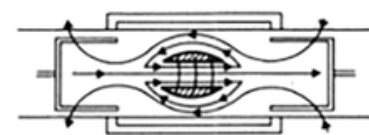

(c)

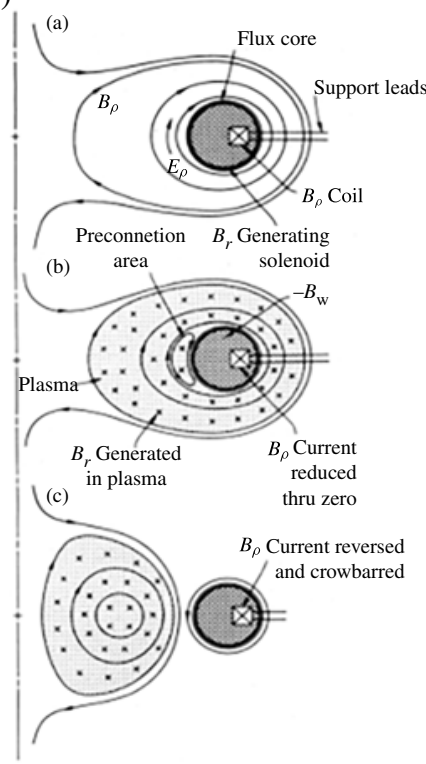

FIGURE 31. Methods to create a spheromak discharge: (a) by plasma gun (reproduced with permission from Turner et al. 1983, copyright 1983, AIP Publishing LLC), (b) by combined $\theta-z$ discharge (reprinted with permission from Goldenbaum et al. 1980, copyright 1980 by the American Physical Society) and (c) by inductive flux core in S-1 (reprinted with permission from Yamada et al. 1981, copyright 1981 by the American Physical Society).

\subsection{Spheromak eigenfunctions}

Axisymmetric eigenfunctions in a spheromak are easily found. The field is again written as (10.1) and the eigenvalue problem reduces to

$$
\Delta^{+} \chi+\mu^{2} \chi=0
$$

with $\chi=0$ on the boundary, as in (10.6). 
In a sphere of radius $a$, the eigenfunction, in spherical coordinates $(\rho, \theta, \phi)$, is

$$
\begin{gathered}
B_{\rho}=2 B_{0}\left[\frac{j_{1}(\mu \rho)}{\mu \rho}\right] \cos \theta, \\
B_{\theta}=B_{0}\left[j_{1}(\mu \rho)\right] \sin \theta, \\
B_{\phi}=-B_{0} \frac{\mathrm{d}}{\mathrm{d} \rho}\left(\rho j_{1}(\mu \rho)\right) \sin \theta,
\end{gathered}
$$

where $j_{1}(x) \equiv \mathrm{J}_{3 / 2}(x) / x^{1 / 2}$, and the eigenvalue is $\mu a=4.49$.

In a cylindrical container of height $h$ and radius $a$ (which, of course, is topologically spherical), the eigenfunction, in cylindrical coordinates $(r, \theta, z)$, is

$$
\begin{gathered}
B_{r}=-B_{0} k \mathrm{~J}_{1}(l r) \cos k z, \\
B_{\phi}=B_{0} \mu \mathrm{J}_{1}(l r) \sin k z, \\
B_{z}=B_{0} k \mathrm{~J}_{0}(l r) \sin k z,
\end{gathered}
$$

where $k h=\pi, l a=3.83$ and the eigenvalue is $\mu^{2}=(3.83 / a)^{2}+(\pi / h)^{2}$.

\subsection{Experimental data}

In figure 32 the poloidal and toroidal field profiles given by (11.8) are compared with those measured in the BETA II experiment (Turner et al. 1983). This has a cylindrical flux conserver with $h / a \sim 1$ and a plasma created by a coaxial gun (see figure $31 a$ ). The agreement is reasonable, particularly in view of the complex way the plasma is created, and it is particularly significant that the measured profiles remain essentially unchanged as the magnetic energy decays to $\sim 1 / 8$ of its initial value. (Recall that the relaxed state of a spheromak is determined solely by the shape of the container.)

The near-uniformity of $\mu$ in a relaxed state is supported by the measurements shown in figure 33, taken on the S-1 spheromak, which has an ellipsoidal flux conserver and a plasma produced by an inductive flux core (Hart et al. 1986). Figure 33(a) shows the ratio of poloidal current to poloidal flux across the mid-plane. The observations lie on a straight line whose slope agrees remarkably well with the computed value of $\mu \sim 5.5$. Figure 33(b) shows how the ratio $J / B=\mu$ on the mid-plane changes from an initial highly non-uniform profile (dashed line) to a much more uniform profile (solid line) after relaxation.

Finally, figure 34 provides the most striking evidence for relaxation. This shows the evolution of the poloidal and toroidal fluxes, and of $q$ on the magnetic axis, during a plasma discharge in the S-1 spheromak (Janos et al. 1985a,b). Figure 34(c) shows that $q$ rises rapidly from a small initial value and becomes close to that predicted for the relaxed state. It is then essentially constant for the remainder of the experiment. This rapid rise in $q$ is accompanied by an increase in toroidal flux (see figure $34 b$ ) and a decrease in poloidal flux (see figure $34 a$ ), during a period when there is strong plasma activity. This again shows that relaxation is not simply a passive decay but involves the creation and destruction of magnetic flux by plasma turbulence - or in this case the conversion of poloidal flux into toroidal flux.

\section{Spherical systems II: flux core spheromak}

An interesting development of the spheromak is a configuration obtained from it by introducing a central core of externally produced magnetic flux along the axis, which enters through one polar cap and leaves through the other (see figure 35). This 'flux core spheromak' again has nested toroidal magnetic surfaces that resemble those in a toroidal pinch, but there is only plasma in the central core and reconnection can occur across the axis. Consequently the toroidal flux is again not conserved in this system. 

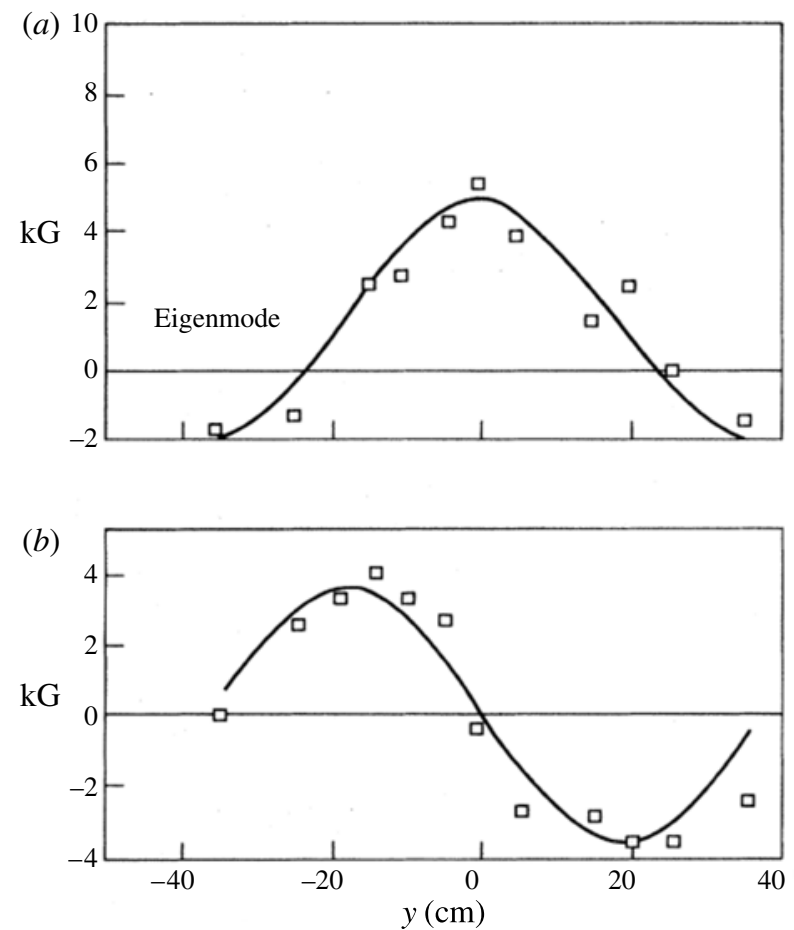

FIgURE 32. Magnetic field profiles in the BETA II spheromak. (a) Poloidal field and (b) toroidal field. Experimental measurements are shown as squares; the solid line is the theoretical prediction. (Reproduced with permission from Turner et al. (1983). Copyright 1983, AIP Publishing LLC.)
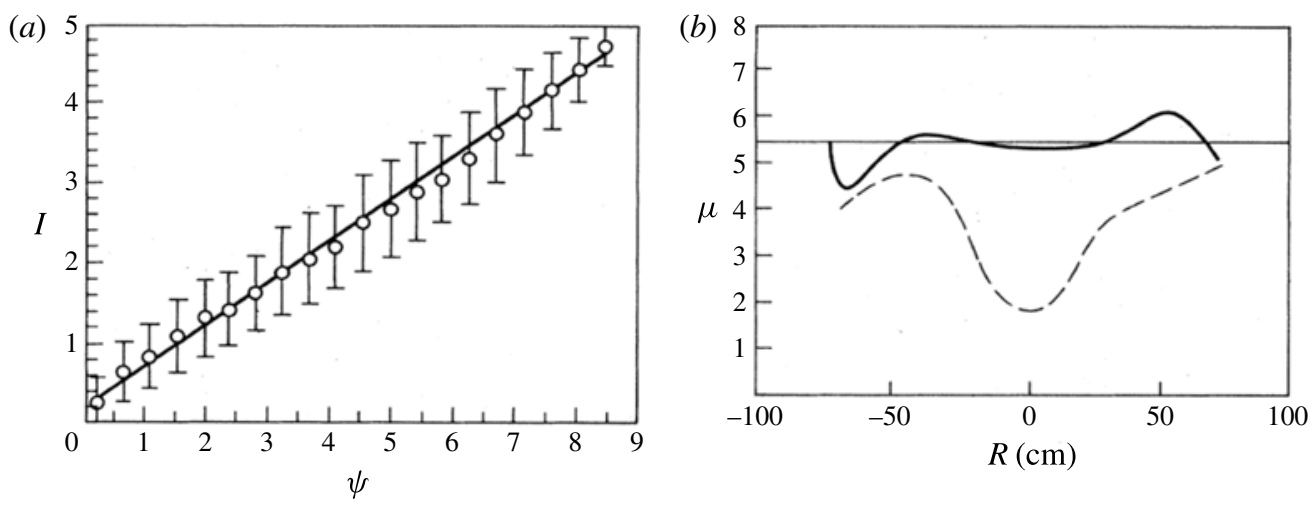

Figure 33. (a) Poloidal current versus poloidal flux and (b) measured profile of $\mu(r)$ in S-1 spheromak. (Reproduced with permission from Hart et al. (1986). Copyright 1986, AIP Publishing LLC.)

\subsection{Relative helicity}

In the flux core spheromak, the plasma boundary is not a completely closed flux surface, and we noted earlier that in this case our definition of helicity is not gaugeinvariant. This is more of a conceptual problem than a practical one, and can be overcome by introducing a modified definition of helicity. 

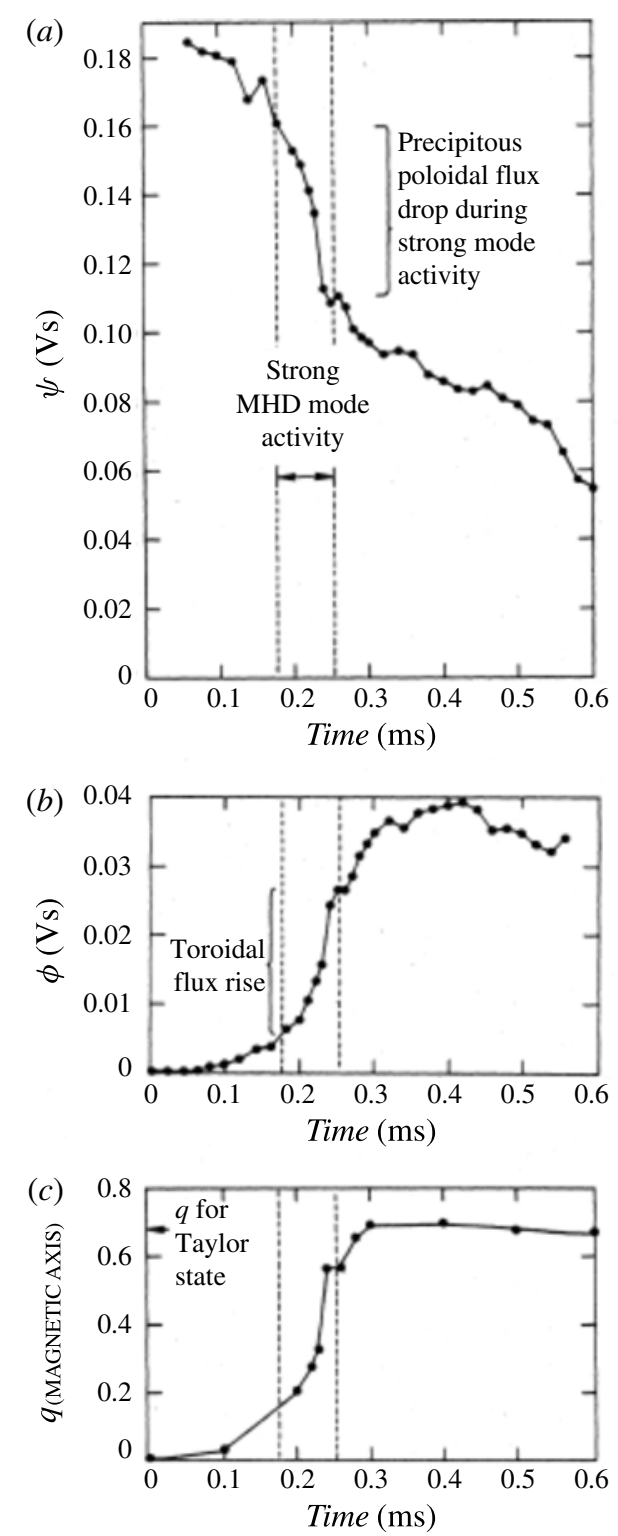

FIGURE 34. Time dependence of magnetic fields in S-1 spheromak - see text for details. (Reproduced with permission from Janos et al. (1985a).)

The most intuitive modification is to imagine that the flux leaving or entering the boundary is extended outside as a vacuum field, where $\nabla \times \boldsymbol{B}=0$. Then the integral $\int \boldsymbol{A} \cdot \boldsymbol{B} \mathrm{d} \tau$, inside plus outside the sphere, is gauge-invariant. If the boundary of the sphere is a perfect conductor, the normal component of $\boldsymbol{B}$ is 'frozen in' and changes inside the sphere do not affect the hypothetical field outside. Therefore, the difference in $\int \boldsymbol{A} \cdot \boldsymbol{B} \mathrm{d} \tau$ for two configurations that differ inside the sphere but have the same $\boldsymbol{n} \cdot \boldsymbol{B}$ on the surface (and therefore the same hypothetical extension field) is also welldefined and gauge-invariant. This is sometimes called the relative helicity, $K_{R}$. 


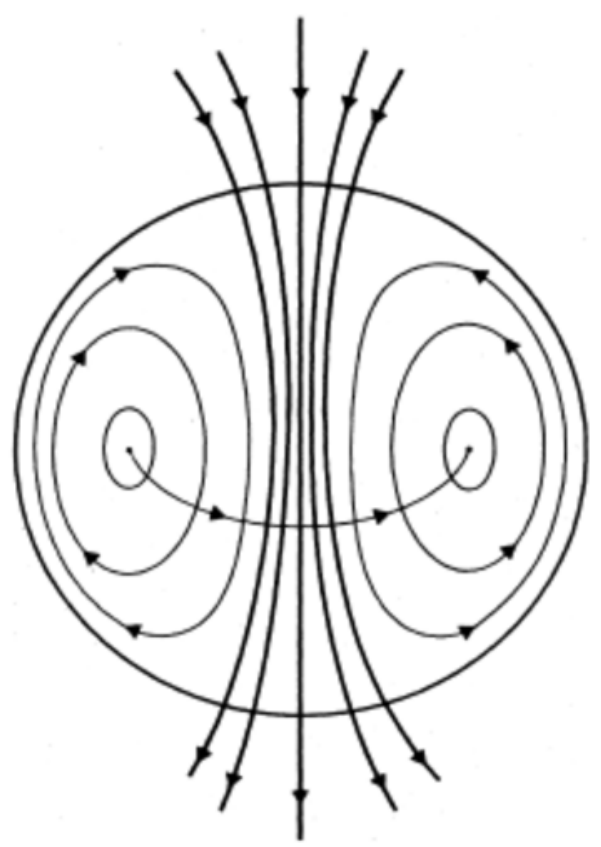

FIGURE 35. Schematic of a flux core spheromak (from Taylor 1986).

Note that $K_{R}$ must include the integral both inside and outside the sphere in order to be gauge-invariant, even though physically the latter does not change. This requirement reflects the fact that helicity is not a local quantity: it can be transferred from inside to outside by a gauge transformation!

The relative helicity satisfies

$$
\frac{\mathrm{d} K_{R}}{\mathrm{~d} t}=-2 \int_{\text {interior }} \boldsymbol{E} \cdot \boldsymbol{B} \mathrm{d} \tau-2 \int_{\text {exterior }} \boldsymbol{E} \cdot \boldsymbol{B} \mathrm{d} \tau .
$$

The exterior field does not change, therefore $\boldsymbol{E}_{\text {exterior }}=-\nabla \phi$ and

$$
\frac{\mathrm{d} K_{R}}{\mathrm{~d} t}=-2 \int_{\text {interior }} \boldsymbol{E} \cdot \boldsymbol{B} \mathrm{d} \tau+2 \oint_{S} \phi \boldsymbol{B} \cdot \mathrm{d} \boldsymbol{S} .
$$

In the interior $\boldsymbol{E}+\boldsymbol{v} \times \boldsymbol{B}=0$ and the first integral vanishes. If the potential $\phi$ is constant over the surface, the last integral is also zero.

The purpose of introducing the flux core spheromak here is that it demonstrates another method of injecting helicity, in this case by a static voltage. If, instead of a uniform potential on the surface, one polar cap is held at a different potential from the other, helicity will be injected at a rate

$$
\frac{\mathrm{d} K_{R}}{\mathrm{~d} t}=2 V_{p} \Psi_{p}
$$

where $V_{p}$ is the voltage between the polar caps and $\Psi_{p}$ is the flux through them (Taylor 1986). 


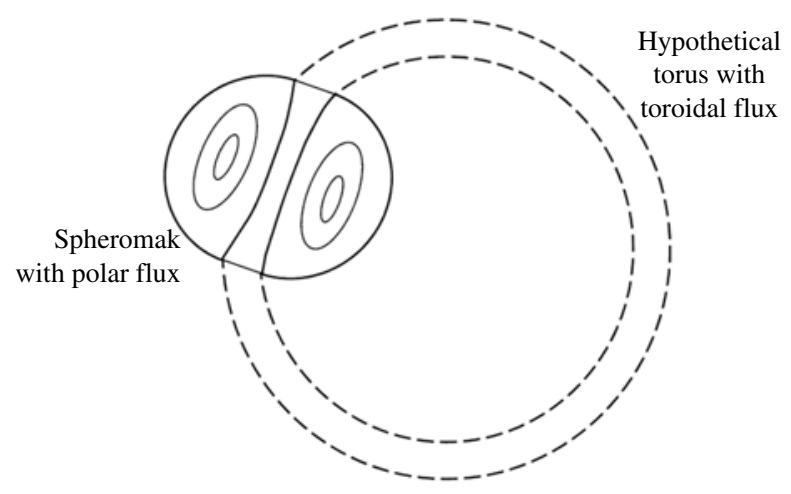

FIGURE 36. Analogy between tokamak and flux core spheromak.

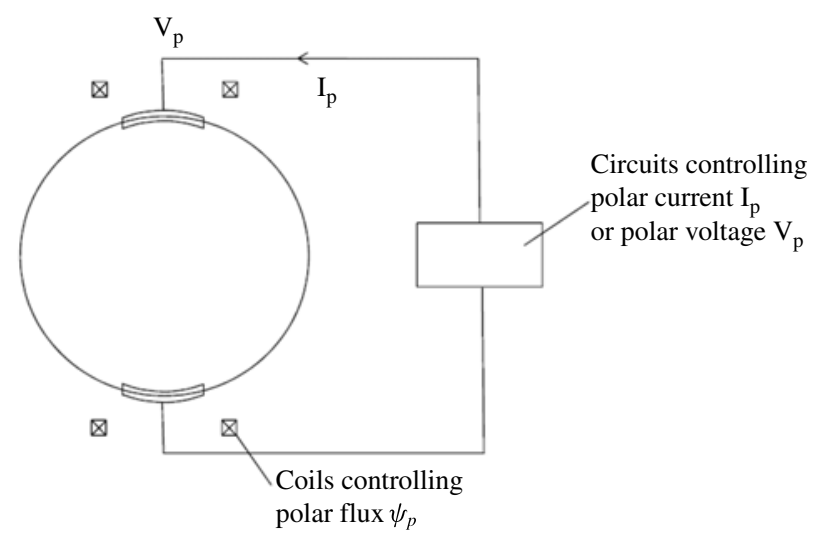

FIGURE 37. Set-up to control relaxed state of flux core spheromak externally.

\subsection{Relaxed states of flux core spheromak}

The relaxed state of the flux core spheromak satisfies the usual equation (11.1) and the relative helicity $K_{R}$ is invariant, but the appropriate solution is selected in a different way. In fact, a flux core spheromak can be operated in several ways. If the plasma relaxes from an initial state with given helicity $K_{R}$, and with fixed flux $\Psi_{p}$ through the polar caps, then $\mu$ is specified by $K_{R} / \Psi_{p}^{2}$ somewhat in the same way as in a toroidal pinch (see figure 36 and $\S 9$ ). However, this is only correct if the polar caps can provide whatever current is required by the resulting relaxed state. If this is not so, a voltage sheath would appear at the polar caps and the resulting voltage drop would reduce the helicity according to (12.3). This leads to another way of operating a flux core spheromak. If one polar cap is insulated from the other and connected to an external circuit that maintains a fixed current $I_{p}$, then in the relaxed state $\mu=I_{p} / \Psi_{p}$. This provides a method for external control of a flux core spheromak (see figure 37).

The relaxed states of a flux core spheromak are calculated in a similar way to those in a simple spheromak. Only the boundary conditions are different. Instead of $\boldsymbol{B} \cdot \boldsymbol{n}=0$ on the boundary, it now has a fixed value on the polar caps and is zero elsewhere. A typical computed field profile is shown in figure 38. Analytic solutions for an idealised flux core system in which the polar caps are shrunk to a point while 


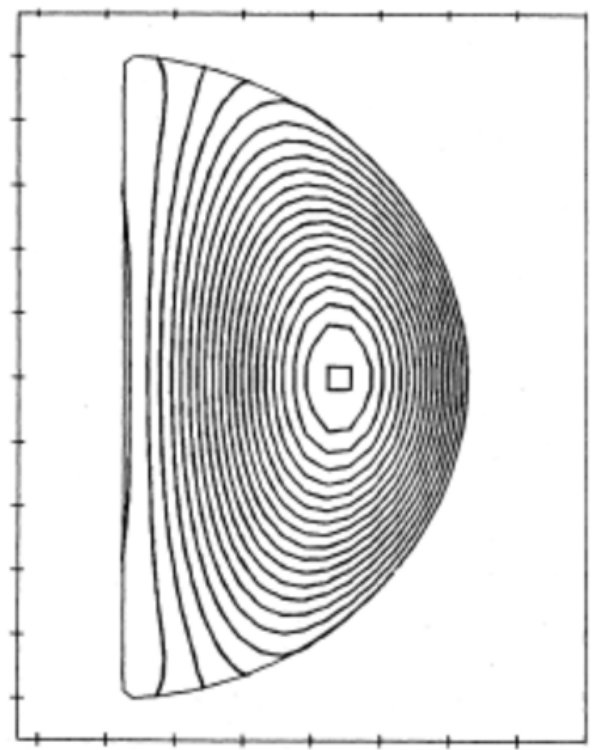

FIGURE 38. Calculated field in a flux core spheromak, $\mu a=4.09$ (from Taylor 1986).

retaining finite flux through them are shown in figure 39. These show the changes that occur as the current through the polar caps, and therefore $\mu$, is increased. When $\mu$ is much less than the lowest spheromak eigenvalue $(\mu a=4.49)$, the externally linked flux and current form a large part of the total flux and current. But as $\mu$ approaches the eigenvalue, the ratio of self-generated flux to externally linked flux increases indefinitely, and the externally linked flux becomes confined to a slim pencil along the axis of symmetry. This situation would constitute an extreme example of the generation of fields by the relaxation process. At the critical point all the fields would, in theory, be generated by the plasma itself.

\subsection{Experimental control of relaxed states}

In a simple spheromak the field in the relaxed state will normally decay at the resistive diffusion rate (while retaining its profile). However, as we noted above, if the polar flux $\Psi_{p}$ and the polar current $I_{p}$ in a flux core spheromak are maintained, it should be possible to maintain its relaxed state at any amplitude and any $\mu=K_{R} / \Psi_{p}^{2}$ below the lowest eigenvalue. This idea is the basis for the experiments described below.

A somewhat distorted form of flux core spheromak, as shown in figure 40, has been extensively investigated at Los Alamos (CTX) (Jarboe et al. 1985; Barnes et al. 1986) and at UMIST (SPHEX) (Kitson \& Browning 1990). The plasma in CTX is created by a plasma gun (on the left of the diagram). This injects plasma into a container, or 'flux conserver', at the right of the figure. If we concentrate attention on the flux conserver, we can see that there is a core of flux (shaded), which passes from the inner electrode of the gun, along the axis of the plasma in the flux conserver and then round the outside of the plasma back to the outer electrode of the gun. From the plasma viewpoint, the gun voltage appears across the flux core and acts as if it were between polar caps. This voltage can sustain the configuration against resistive decay for many times the usual resistive diffusion time. 


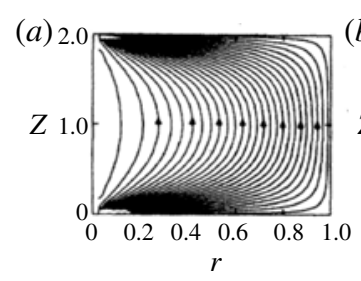

(6)

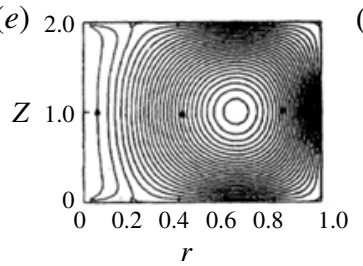

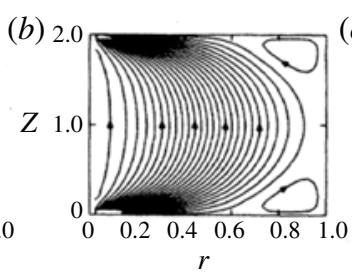

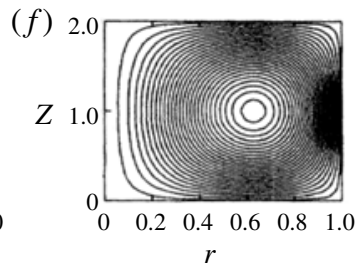

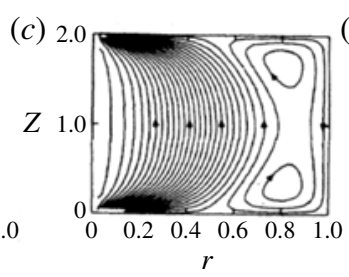

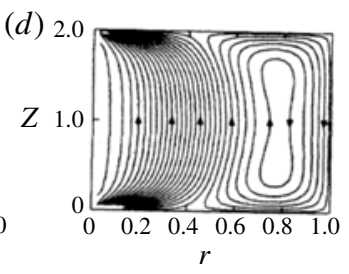

(g) 2.0

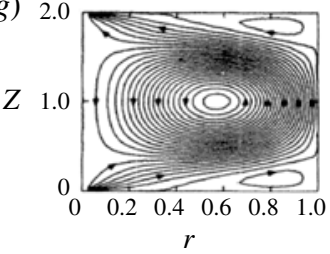

FIGURE 39. Evolution of analytic field profiles in an idealised flux core spheromak at various values of $\mu a$ : (a) $\mu a=0.001,(b) \mu a=2.25,(c) \mu a=2.70,(d) \mu a=3.00,(e) \mu a=$ 4.00, $(f) \mu a=4.14$ and $(g) \mu a=5.00$. (Reproduced with permission from Turner (1984). Copyright 1984, AIP Publishing LLC.)

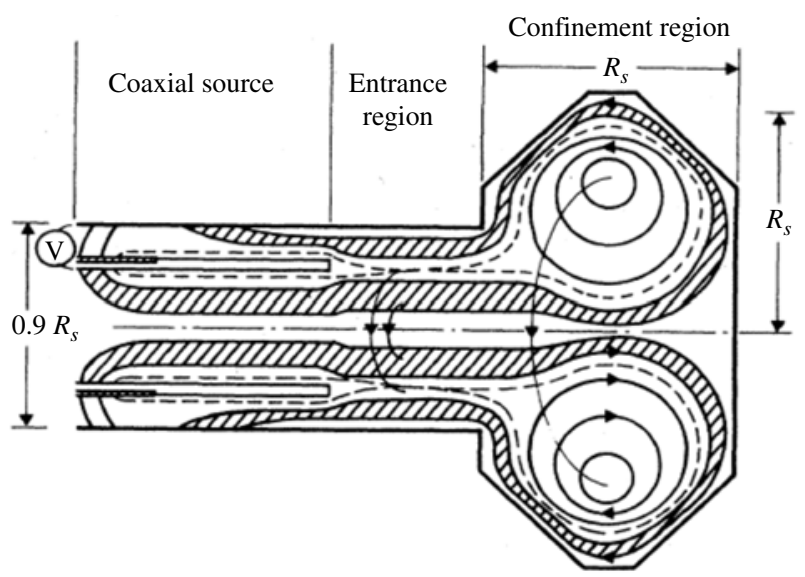

FIGURE 40. Sustained configuration in CTX experiment (from Jarboe et al. 1985).

At this point we should note the time scales involved in this discussion:

(i) Relaxation time. This is much faster than the resistive diffusion time $a^{2} / \eta$, and often close to the Alfvén time. This leads to the relaxed state. The helicity $K_{R}$ is invariant on this time scale.

(ii) The resistive diffusion time $\sim a^{2} / \eta$. This is the time scale on which $K_{R}$ and the relaxed state itself decays, if it is not maintained by some external means.

(iii) The sustainment time. This is the time, longer than $a^{2} / \eta$, on which the discharge can be maintained in its relaxed state by an external voltage.

\subsection{The kinked Z-pinch}

Once it was realised that a flux core spheromak could be maintained in the ways described above, it was found that they could also be created and maintained in more 


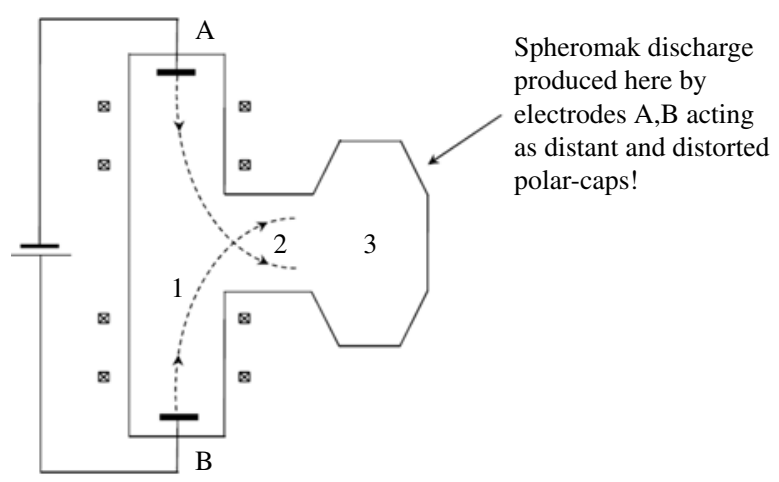

FIGURE 41. Illustration of kinked Z-pinch source.

exotic configurations. One of these was the 'kinked Z-pinch' helicity source (Jarboe et al. 1985; Fernández et al. 1989), shown in figure 41. In a highly idealised way, we can regard this device as creating a sequence of relaxed states in each of the three regions marked 1, 2 and 3. In region 1, we have a simple linear pinch maintained by the electrodes. Insofar as it is relaxed, $\mu$ would be set by the ratio of the current $I_{s}$ and flux $\Psi_{s}$ at the electrodes, $\mu_{1}=I_{s} / \Psi_{s}$. In region 2, we have a helical relaxed state of a circular cylinder, with no net current or flux. If the cylinder were long, this would be the $m=1, k a=1.23$ eigenfunction, which we discussed in connection with the cylindrical pinch. This would have $\mu_{2} a_{2} \sim 3.11$ as calculated in $\S 9.1$. Finally, in region 3 , we would have a spheromak with $\mu_{3} a_{3}$, defined solely by the size and shape of the flux conserver (see $\$ 11.2$ ).

Now, recall from $\S 9.1$ that the energy for a given helicity in a relaxed state increases with $\mu$. Consequently, it will be energetically favourable for the discharge to pass from region 1 to 2 and then into 3 if, and only if,

$$
\mu_{1}>\mu_{2}>\mu_{3}
$$

In fact, $\mu_{2}>\mu_{3}$ is set by the dimensions of the apparatus, but $\mu_{1}$ can be controlled externally and we can expect there to be a threshold for the successful injection of the spheromak into the flux conserver, set by

$$
\frac{I_{s}}{\Psi_{s}}=\mu_{1}>\mu_{2} .
$$

Such a threshold does seem to occur, as can be seen in figure 42, which shows a 'figure of merit' for the spheromak created in the flux conserver. This is roughly proportional to its total helicity. Figure $42(a)$ is for a $20 \mathrm{~cm}$ radius entrance cylinder, whilst figure $42(b)$ is for a $17 \mathrm{~cm}$ radius entrance cylinder. It is clear from these figures that there is no significant helicity input into the flux conserver unless $\mu_{1}>$ $13 \mathrm{~m}^{-1}$ in $(a)$, and $\mu_{1}>14 \mathrm{~m}^{-1}$ in $(b)$. The theoretical threshold for a long cylinder would be $\sim 15.6 \mathrm{~m}^{-1}$ for a $20 \mathrm{~cm}$ entrance radius.

\section{Helicity injection}

As we have seen in several situations, because a relaxed state is completely determined by the helicity $K$ and flux $\Psi$, it can be maintained for longer than the resistive decay time if $K$ and $\Psi$ can be controlled externally. 

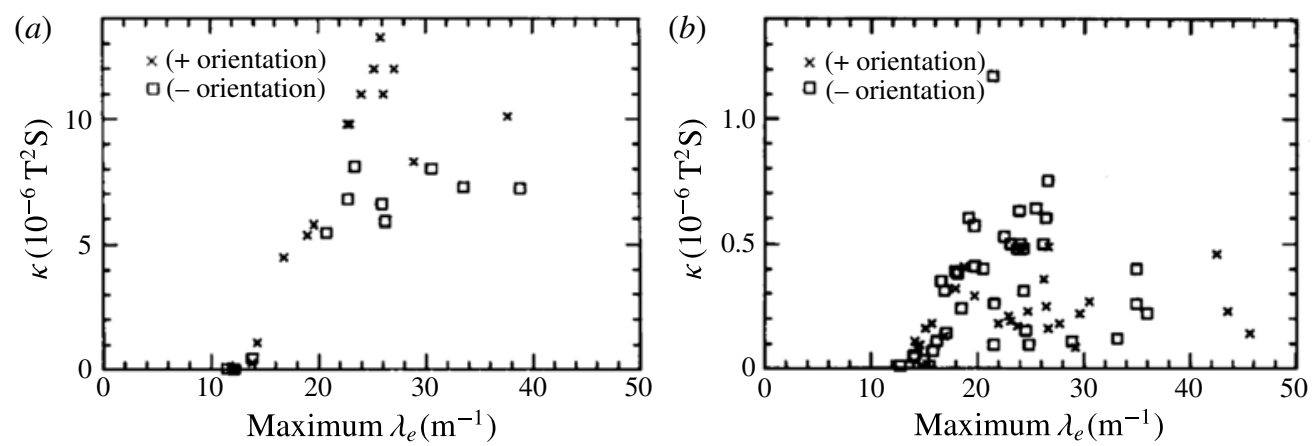

FIGURE 42. Spheromak figure of merit $\kappa$ versus $\lambda_{e} \equiv \mu$. (Reproduced with permission from Fernández et al. (1989). Copyright 1989, AIP Publishing LLC.)

In a toroidal system there is no difficulty in principle maintaining $\Psi_{\text {tor }}$ by the usual toroidal field coils, and in (7.25) we observed that

$$
\frac{\mathrm{d} K}{\mathrm{~d} t}=2 V_{\text {loop }} \Psi_{\text {tor }},
$$

apparently giving control over $K$. But unfortunately $V_{\text {loop }}$ itself can be maintained only for a short time - limited by the volt-seconds available from the driving transformer. However, an oscillating $V_{\text {loop }}$ could be maintained, and if $\Psi_{\text {tor }}$ is simultaneously modulated in the appropriate phase, there is an average input of helicity

$$
\left\langle\frac{\mathrm{d} K}{\mathrm{~d} t}\right\rangle \sim \hat{V}_{\text {loop }} \hat{\Psi}_{\text {tor }} \cos \theta,
$$

where $\hat{V}_{\text {loop }}$ and $\hat{\Psi}_{\text {tor }}$ are the oscillation and modulation amplitudes, and $\theta$ is the phase difference between them.

If this method is to have any chance of success, the plasma relaxation time must be less than the period of oscillation (so that the plasma stays close to its relaxed state) and the period of oscillation must be much less than the resistive decay time, so that there is negligible decay between each cycle.

This method of helicity injection, which is also known as 'oscillating field current drive' (OFCD), was attempted on the ZT-40M experiment (Schoenberg et al. 1988) with limited success. In part this was due to the small amplitudes of $V_{\text {loop }}$ and $\Psi_{\text {tor }}$ that were available, but a more fundamental obstacle is that the process disturbs the plasma equilibrium and creates serious losses by 'modulation-enhanced plasma-wall interaction'.

In a flux core spheromak we saw that a voltage $V_{p}$ applied to the polar caps injects helicity at a rate

$$
\frac{\mathrm{d} K}{\mathrm{~d} t}=2 V_{p} \Psi_{p}
$$

where $\Psi_{p}$ is the flux linking the polar caps. This method of injection was successfully used in the CTX and other experiments, described in $\S 12.3$.

\subsection{Spheromak injection into tokamak}

A remarkable application of helicity injection is the attempt (Brown \& Bellan 1990) to influence the current in a tokamak byinjecting helicity in the form of small 


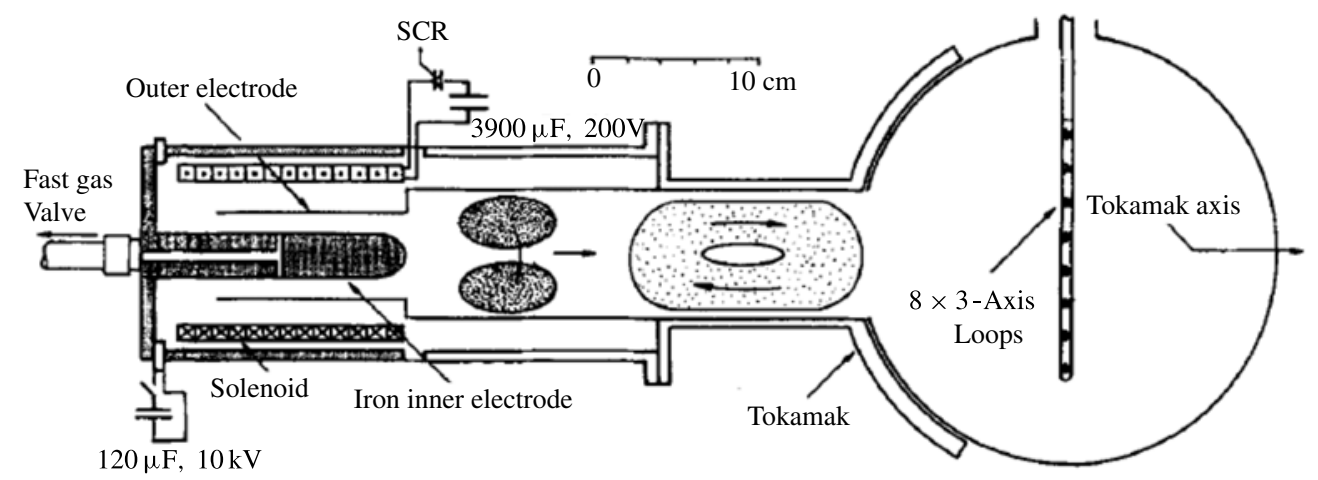

FIGURE 43. Schematic of spheromak injection into ENCORE tokamak at Caltech. (Reproduced with permission from Brown \& Bellan (1990). Copyright 1990, AIP Publishing LLC.)

spheromaks from a coaxial gun in a side arm into the main torus! This is illustrated in figure 43. As in the previous discussions, a small spheromak is created in the mouth of the gun and moves into the main tokamak chamber. To understand the observations, one must recall that helicity is a pseudo-scalar and therefore can be right-handed or left-handed. The handedness is related to the sign of $\mu$, that is, to the relative directions of $\boldsymbol{J}$ and $\boldsymbol{B}$.

One can set up the main tokamak discharge with either right- or left-handed helicity by reversing the direction of the toroidal magnetic field, and one can create spheromaks with either right- or left-handed helicity by reversing the polarity of the gun voltage. Therefore, there are four permutations of the experiment:

(a) L-hand spheromak injected into L-hand tokamak,

(b) L-hand spheromak injected into R-hand tokamak,

(c) R-hand spheromak injected into R-hand tokamak,

(d) R-hand spheromak injected into L-hand tokamak.

The effect on the tokamak current of injecting the spheromak for the four cases is shown in figure 44. The dotted line in (a) shows the behaviour when there is no spheromak injection. It can be seen that when the injected spheromak and the tokamak have the same chirality (cases (a) and (c)), there is a small spike in the tokamak current. Conversely, when the spheromak and tokamak have opposite helicity (cases (b) and (d)), there is a small drop in the tokamak current. In all four cases the initial rise or drop in current is followed by a rapid decay. This is thought to be due to the cooling of the main plasma by the large amount of cold gas that accompanies the spheromak from the coaxial gun.

\subsection{Wave injection}

Another, untried, method of helicity injection is by plasma waves (Mett \& Tataronis 1989; Taylor 1989; Mett \& Taylor 1992). One might envisage doing this by means of an antenna similar to those used for plasma heating.

Helicity injection by waves is hard to visualise (but see Chan et al. 1990). We have previously emphasised that helicity is a non-local quantity - the linking of lines of force - and it is difficult to relate this to wave motion. In fact, this process is more 


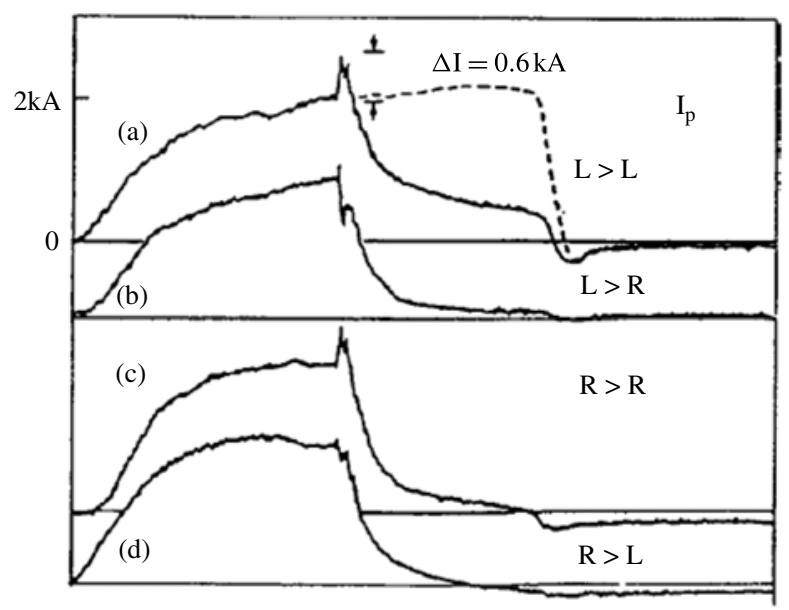

FIGURE 44. Effect on tokamak current of spheromak injection - see text for details. (Reproduced with permission from Brown \& Bellan (1990). Copyright 1990, AIP Publishing LLC.)

usually described as 'current drive', but it is interesting to see the connection with helicity. There are, of course, many types of plasma wave, but we will consider only the simplest - waves in a uniform plasma immersed in a uniform magnetic field $\boldsymbol{B}=$ $\left(0,0, \boldsymbol{B}_{0}\right)$. However, this already illustrates some of the conceptual problems.

We start from what looks like a continuity equation for $\boldsymbol{A} \cdot \boldsymbol{B}$,

$$
\frac{\partial}{\partial t}(\boldsymbol{A} \cdot \boldsymbol{B})+\nabla \cdot \boldsymbol{Q}+2(\boldsymbol{E} \cdot \boldsymbol{B})=0
$$

where $\boldsymbol{Q}=\phi \boldsymbol{B}+\boldsymbol{E} \times \boldsymbol{A}$. For a plasma with an Ohm's law $\boldsymbol{E}+\boldsymbol{v} \times \boldsymbol{B}=\eta \boldsymbol{j}$,

$$
\frac{\partial}{\partial t}(\boldsymbol{A} \cdot \boldsymbol{B})+\nabla \cdot \boldsymbol{Q}+2 \eta(\boldsymbol{j} \cdot \boldsymbol{B})=0 .
$$

Neither $\boldsymbol{A} \cdot \boldsymbol{B}$ nor $\boldsymbol{Q}$ is gauge-invariant, but it is clear that $\boldsymbol{Q}$ must represent a flux of helicity. If we consider a small-amplitude wave so that $\boldsymbol{B}=\boldsymbol{B}_{0}+\boldsymbol{b} \mathrm{e}^{\mathrm{i} \omega t}, \boldsymbol{Q}$ becomes

$$
\boldsymbol{q}=\frac{1}{2}\left[\phi \boldsymbol{b}^{*}+\left(\boldsymbol{e} \times \boldsymbol{a}^{*}\right)+\text { c.c. }\right],
$$

and we can therefore introduce a flux

$$
\boldsymbol{q}^{\prime}=\frac{\mathrm{i}}{\omega}\left(\boldsymbol{e} \times \boldsymbol{e}^{*}\right),
$$

which has the same divergence as $\boldsymbol{q}$ but is manifestly gauge-invariant. This form also brings out the connection between helicity and polarisation: a plane-polarised wave carries no helicity. (Actually this is obvious, as a plane-polarised wave has complete mirror symmetry whereas helicity is antisymmetric under reflection.)

However, a circularly polarised wave, propagating parallel to $\boldsymbol{B}_{0}$, with a vector potential given by the real part of

$$
\boldsymbol{A}=(A, \mathrm{i} A, 0) \exp [\mathrm{i}(k z-\omega t)]
$$


does carry helicity in the form (13.7). (Here $\omega$ is real and $k$ complex, $k=k_{0}(\omega)+$ $\mathrm{i} k_{1}(\omega)$, as for waves generated by an antenna at $z=0$.)

From (13.8)

$$
\boldsymbol{e}=(\mathrm{i} \omega A,-\omega A, 0) \exp [\mathrm{i}(k z-\omega t)]
$$

therefore

$$
\boldsymbol{q}^{\prime}=\boldsymbol{q}_{z}=\omega|A|^{2} \mathrm{e}^{-2 k_{1} z}
$$

Define $|A|^{2} \mathrm{e}^{-2 k_{1} z}=A^{2}(z)$, the square of the local wave amplitude, then

$$
\boldsymbol{q}_{z}=\omega A^{2}(z)=v\left(k_{0} A^{2}\right),
$$

where $v=\omega / k_{0}$ is the wave velocity. This can be interpreted as the wave carrying helicity density $k_{0} A^{2}$. Finally, then,

$$
\nabla \cdot \boldsymbol{q}=-2 \omega k_{1} A^{2}(z) .
$$

Now consider a specific plasma wave, an Alfvén wave in a resistive plasma, with viscosity $v$. The linearised wave equations are

$$
\begin{gathered}
-\omega \boldsymbol{b}=(\boldsymbol{k} \cdot \boldsymbol{B}) \boldsymbol{v}+\mathrm{i} \frac{\eta}{\mu_{0}} k^{2} \boldsymbol{b}, \\
-\omega \rho \boldsymbol{v}=\frac{1}{\mu_{0}}(\boldsymbol{k} \cdot \boldsymbol{B}) \boldsymbol{b}+\mathrm{i} \rho \nu k^{2} \boldsymbol{v} .
\end{gathered}
$$

This has the dispersion relation

$$
\left(\omega+\mathrm{i} \frac{\eta}{\mu_{0}} k^{2}\right)\left(\omega+\mathrm{i} \nu k^{2}\right)=k^{2} v_{A}^{2},
$$

where $v_{A}$ is the Alfvén speed (see (4.5)). When $\eta$ and $v$ are small, $k_{0} \approx \omega / v_{A}$ and the wave damping is $k_{1} \approx\left(\eta \mu_{0}^{-1}+v\right) k_{0}^{2} / 2 v_{A}$. Therefore

$$
\boldsymbol{\nabla} \cdot \boldsymbol{q}=-\left(\frac{\eta}{\mu_{0}}+v\right) k_{0}^{3} A^{2}(z) .
$$

Thus it appears that we can indeed inject helicity from polarised waves, and that the input is proportional to the total wave damping, $\left(\eta \mu_{0}^{-1}+v\right)$.

As mentioned above, this form of helicity injection is conventionally interpreted as current drive. The mean current induced by the wave fluctuations can be calculated directly from Ohm's law $\eta\left\langle j_{z}\right\rangle=\langle\boldsymbol{v} \times \boldsymbol{b}\rangle$. If the wave equation (13.14) is used to express $\boldsymbol{v}$ in terms of $\boldsymbol{b}$, then

$$
\eta\left\langle j_{z}\right\rangle=\frac{A^{2}(z)}{B_{0}}\left(\omega k_{1}-\frac{\eta}{\mu_{0}} k^{2} k_{0}\right)
$$

and using $k_{1}$ as given above, the wave-driven current is

$$
\eta\left\langle j_{z}\right\rangle=\frac{k_{0}^{3} A^{2}(z)}{2 B_{0}}\left(v-\frac{\eta}{\mu_{0}}\right) .
$$

This is in agreement with the net helicity injection given by (13.4) in steady state,

$$
-\nabla \cdot \boldsymbol{q}-2 \eta\left\langle\boldsymbol{j}_{\omega} \cdot \boldsymbol{b}_{\omega}\right\rangle=\left[\left(\eta \mu_{0}^{-1}+v\right)-2 \eta \mu_{0}^{-1}\right] k_{0}^{3} A^{2}(z)=\left(v-\eta \mu_{0}^{-1}\right) k_{0}^{3} A^{2}(z),
$$

where $2 \eta\left\langle\boldsymbol{j}_{\omega} \cdot \boldsymbol{b}_{\omega}\right\rangle$ is the decay of helicity associated with the wave fluctuations (13.8) themselves. Note the remarkable fact that, if resistivity were the only damping, the loss of helicity through the wave fluctuations would be exactly twice the input from the wave. 


\section{General theory of relaxed toroidal states}

It is interesting to cast the theory of relaxed states in a general form that illustrates the connection between different types of relaxed state (Jensen \& Chu 1984; Taylor 1986). (Even though it is not a convenient way to study specific examples.)

In a general toroidal system, the relaxed state is the lowest-energy, that is, smallest$\mu$, solution of

$$
\nabla \times(\nabla \times A)=\mu \nabla \times A
$$

with $\boldsymbol{n} \cdot \boldsymbol{\nabla} \times \boldsymbol{A}=0$ on the boundary and $\oint \boldsymbol{A} \cdot \mathrm{d} \boldsymbol{l}, \oint \boldsymbol{A} \cdot \mathrm{d} \boldsymbol{s} \equiv \Psi$ and $\int \boldsymbol{A} \cdot \boldsymbol{B} \mathrm{d} \tau \equiv K$ specified. (The use of $\boldsymbol{A}$ instead of $\boldsymbol{B}$ allows all constraints to be set as boundary conditions.)

To describe the possible relaxed states, we must also introduce the eigenfunctions defined by

$$
\nabla \times\left(\nabla \times a_{i}\right)=\lambda_{i} \nabla \times \boldsymbol{a}_{i},
$$

with boundary condition $\boldsymbol{a}_{i}=0$. Note that the eigenfunctions carry no toroidal flux.

As we found explicitly in the case of the circular cylinder, the relaxed state is one of two possibilities:

(i) a primitive solution of (14.1) in which $\mu$ is determined by $K / \Psi^{2}$;

(ii) a mixed solution, that is, a superposition of a primitive solution and an eigenfunction, in which case $\mu$ is determined as the smallest eigenvalue and $K / \Psi^{2}$ determines the relative amplitudes of the eigenfunction and primitive components.

To see what differences, if any, might arise in a general toroidal system, we assume that the $\boldsymbol{a}_{i}$ are a sufficiently complete set and write the solution as

$$
\boldsymbol{A}=\boldsymbol{A}_{0}+\sum \alpha_{i} \boldsymbol{a}_{i}
$$

where $\boldsymbol{A}_{0}$ is the vector potential for a vacuum field that satisfies the same boundary conditions as the actual problem. The eigenfunctions are orthogonal, where the following integrals are taken over the system volume:

$$
\left(\lambda_{i}-\lambda_{j}\right) \int \boldsymbol{a}_{i} \cdot \nabla \times \boldsymbol{a}_{j} \mathrm{~d} \tau=0
$$

and

$$
\lambda_{i} \int \boldsymbol{a}_{i} \cdot \nabla \times \boldsymbol{a}_{i} \mathrm{~d} \tau=\int\left(\nabla \times \boldsymbol{a}_{i}\right)^{2} \mathrm{~d} \tau>0,
$$

so they can be normalised such that

$$
\int \boldsymbol{a}_{i} \cdot \nabla \times \boldsymbol{a}_{j} \mathrm{~d} \tau=\int \boldsymbol{a}_{j} \cdot \nabla \times \boldsymbol{a}_{i} \mathrm{~d} \tau=\frac{\lambda_{i}}{\left|\lambda_{i}\right|} \delta_{i j} .
$$

Substituting (14.3) into the relaxed-state equation (14.1), and using the orthogonality of the eigenfunctions, we can find the $\alpha_{i}$ - provided $\mu$ is not an eigenvalue. Then

$$
\begin{gathered}
\boldsymbol{A}=\boldsymbol{A}_{0}+\sum_{i} \frac{\mu}{\left(\lambda_{i}-\mu\right)} T_{i} \boldsymbol{a}_{i}, \\
T_{i} \equiv \int \boldsymbol{a}_{i} \cdot \nabla \times \boldsymbol{A}_{0} \mathrm{~d} \tau
\end{gathered}
$$




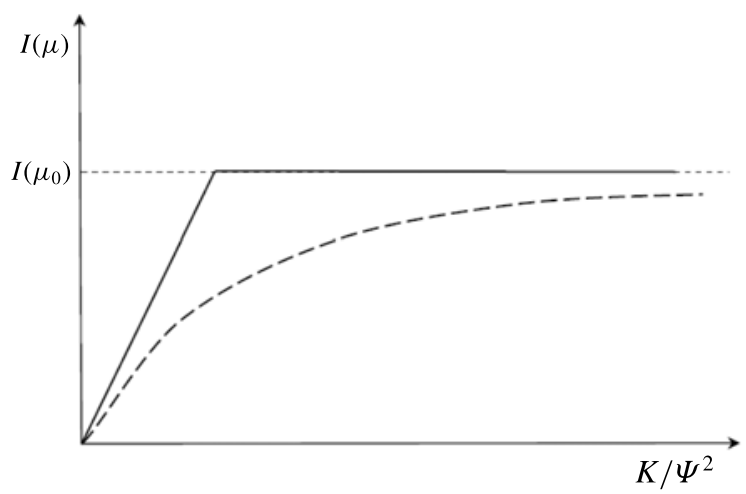

FIGURE 45. Current saturation in general relaxed states: soft (dashed) and hard (solid). Lowest eigenvalue is $\mu_{0}$.

and

$$
\frac{K}{\Psi^{2}}=\frac{1}{\Psi^{2}}\left\{\int\left(\boldsymbol{A}_{0} \cdot \nabla \times \boldsymbol{A}_{0}\right) \mathrm{d} \tau+\sum_{i} T_{i}^{2} \frac{\lambda_{i}}{\left|\lambda_{i}\right|}\left(\frac{\lambda_{i}^{2}}{\left(\lambda_{i}-\mu\right)^{2}}-1\right)\right\}
$$

This defines $\mu$ as $\mu=f\left(K / \Psi^{2}\right)$. We are interested only in the smallest $\mu$, which must lie below $\lambda_{0}$ because $K / \Psi^{2} \rightarrow \infty$ as $\mu \rightarrow \lambda_{0}$.

However, many eigenfunctions may be decoupled from the vacuum field, that is

$$
T_{i}=\int \boldsymbol{a}_{i} \cdot \nabla \times \boldsymbol{A}_{0} \mathrm{~d} \tau=0 .
$$

For example, in an axisymmetric (but not necessarily circular cross-section) system, all the non-axisymmetric eigenfunctions $\sim \exp (\mathrm{in} \phi)$ are decoupled from the axisymmetric vacuum field. Some axisymmetric eigenfunctions may also be decoupled, e.g. by the (anti)symmetry about the equatorial plane, as in the multipinch. To remind us of this we write

$$
\boldsymbol{A}=\boldsymbol{A}_{0}+\sum_{i}^{\prime} \frac{\mu}{\left(\lambda_{i}-\mu\right)} \frac{\lambda_{i}}{\left|\lambda_{i}\right|} T_{i} \boldsymbol{a}_{i}
$$

where $\sum_{i}^{\prime}$ denotes the sum only over coupled eigenfunctions.

If the lowest eigenfunction (that with the smallest $\left.\lambda_{0}\right)$ is not decoupled $\left(T_{0} \neq 0\right)$, the relaxed state (14.7) exhibits a 'soft' form of current saturation in which the toroidal current $I \rightarrow I_{\text {sat }}$ as $K / \Psi^{2} \rightarrow \infty$ (see (14.9) and the dashed line in figure 45).

If the lowest eigenfunction $\boldsymbol{a}_{0}$ is decoupled, then when $\mu=\lambda_{0}$ we can add a term $\beta \boldsymbol{a}_{0}$ to the sum (14.11) (which otherwise does not contain $\boldsymbol{a}_{0}$ ) to create a state in which $\beta$ is determined by $K / \Psi^{2}$. This state exhibits a 'hard' saturation in which the toroidal current abruptly stops increasing when $K / \Psi^{2}$ reaches a finite critical value (solid line in figure 45).

At this point one should ask the following questions. What if, as is always the case in the real world, there are small field errors in a supposedly axisymmetric system, which couple the supposedly decoupled $\boldsymbol{a}_{0}$ to the vacuum field? Does one then get a primitive state with soft saturation or a mixed state with hard saturation? 
In fact, there is no anomaly. If small field errors are incorporated, we have

$$
\boldsymbol{A}=\boldsymbol{A}_{0}+\sum_{i}^{\prime} \frac{\mu}{\left(\lambda_{i}-\mu\right)} \frac{\lambda_{i}}{\left|\lambda_{i}\right|} T_{i} \boldsymbol{a}_{i}+\frac{\mu}{\left(\lambda_{0}-\mu\right)} \frac{\lambda_{0}}{\left|\lambda_{0}\right|} \tilde{T}_{0} \boldsymbol{a}_{0},
$$

where $\tilde{T}_{0}$ is the coupling to the error field, and if the error field is ignored we have

$$
\boldsymbol{A}=\boldsymbol{A}_{0}+\sum_{i}^{\prime} \frac{\mu}{\left(\lambda_{i}-\mu\right)} \frac{\lambda_{i}}{\left|\lambda_{i}\right|} T_{i} \boldsymbol{a}_{i}+\beta \boldsymbol{a}_{0} .
$$

Taking the view that we should include error fields, if we let these decrease so that $\tilde{T}_{0} \rightarrow 0$, then we find that (for fixed $\left.K / \Psi^{2}\right) \mu \rightarrow \lambda_{0}$ and the limit $\tilde{T}_{0} \lambda_{0} \mu /\left|\lambda_{0}\right|\left(\mu_{0}-\mu\right)$ $\rightarrow \beta$, so we have the same result as when field errors are ignored. Consequently, the soft saturation with field errors simply goes over to the hard saturation as field errors diminish (see figure 45).

\section{Conclusion}

The theory of relaxation has been remarkably successful in accounting for, and predicting, the behaviour of turbulent plasmas in many different circumstances. Even more remarkable is that it does this from 'first principles' - there are no fitted or arbitrary parameters in the calculation of relaxed states.

It is therefore important to be clear about the scope and limits of the theory. Some significant points are:

(i) Relaxation is a theory of turbulent plasma. It is turbulence, allied to a small resistivity, that enables the plasma to reach the relaxed state. A plasma, or a simulation, that is at all times stable or quiescent will not relax.

(ii) Because relaxation depends on turbulence, there is always some uncertainty whether relaxation will be complete. This is particularly relevant in tokamak experiments where there is a strong stabilising vacuum magnetic field, unlike the experiments we have discussed in which the magnetic field is largely generated by currents in the plasma. (Currents in the plasma are free to move; those in massive external metal coils are not supposed to!) Also, tokamaks are carefully designed and operated to maintain stability as far as possible. Consequently it appears that relaxation in tokamaks is only partial and limited, as in sawteeth and disruptions - and even these are avoided as much as possible.

(iii) Relaxation theory is concerned with actual plasma motions. Despite its mathematical similarity, it should not be confused with purely mathematical minimisation principles involving virtual displacements, such as Hamilton's principle. Nor should one arbitrarily add extra conserved mathematical quantities unless there is a physical reason to do so. The cornerstone of the theory is that turbulence plus resistivity introduces localised reconnection that scarcely changes total helicity, but has a global effect on plasma behaviour.

(iv) Relaxation theory says nothing about exactly how the plasma gets to the relaxed state. (This is both its great strength and its weakness.) It describes only the state the plasma eventually (though very rapidly compared to resistive processes) reaches. In this regard it is similar to a statistical derivation of thermal distributions, which also does not describe in detail how this distribution is reached. 
(v) You will have noticed that we have said nothing about plasma pressure and that all relaxed states are force-free, stable, equilibria. This is because, if lines of force are free to break and reconnect, we must expect that pressure will be equalised by flow along the field during this reconnection. One might suggest that this equalisation would be slower than reconnection, but to describe it would need a full theory of plasma turbulence capable of calculating the development from initial to final state. Not only do we lack such a theory, but it would not be in any way universal - which was the original requirement of a theory to explain the quiescent states.

(vi) In the historical development of confinement systems a distinction was initially drawn between (1) those like mirrors and stellarators, in which one could envisage setting up an unquestionably stable vacuum field and adding plasma as a perturbation, and (2) those like toroidal pinches, in which the plasma itself produced much of the confining field and which therefore lacked the obvious stability of a vacuum field. One might claim that the discovery of relaxed states somewhat reduced this distinction, with the relaxed state playing the role of the vacuum field.

\section{Part III}

\section{Adiabatic traps and mirror machines}

So far, we have concentrated on toroidal confinement systems and the fluid model. Now we turn to a different confinement system - the adiabatic trap - and the guiding centre model.

In an adiabatic trap, the magnetic field lines are not confined to the system. (Recall that this is only possible in a torus.) Instead, plasma loss along the field lines is inhibited by the 'magnetic mirror' effect. This depends on the existence of an 'adiabatic invariant' for each particle - its magnetic moment $\mu=m v_{\perp}^{2} / 2 B$.

The invariance of $\mu$, together with the fact that the total energy, $E$, of a particle is constant in a static field, means that

$$
\frac{1}{2} m v_{\|}^{2}=E-\mu B,
$$

so that $\mu B$ acts as a potential for the parallel motion. Particles in a field $B_{0}$ are therefore 'reflected' by a stronger field $B_{m}$ if their velocity lies outside the 'loss cone'

$$
\left(\frac{v_{\perp}}{v}\right)<\left(\frac{B_{0}}{B_{m}}\right)^{1 / 2}
$$

A typical mirror machine based on this effect is shown in figure 46.

Note that the existence of a loss cone in velocity space means that the plasma pressure in an adiabatic trap is always anisotropic. This is an important distinction between adiabatic traps and toroidal systems - which usually have near-isotropic pressure. It also means that scattering into the loss cone inevitably leads to loss of plasma in mirror machines.

\subsection{Digression on adiabatic invariants}

Adiabatic invariants are an interesting feature of classical mechanics and were important in the 'old' quantum theory - where they were the classical variables that became 'quantised', i.e. restricted to discrete values. 


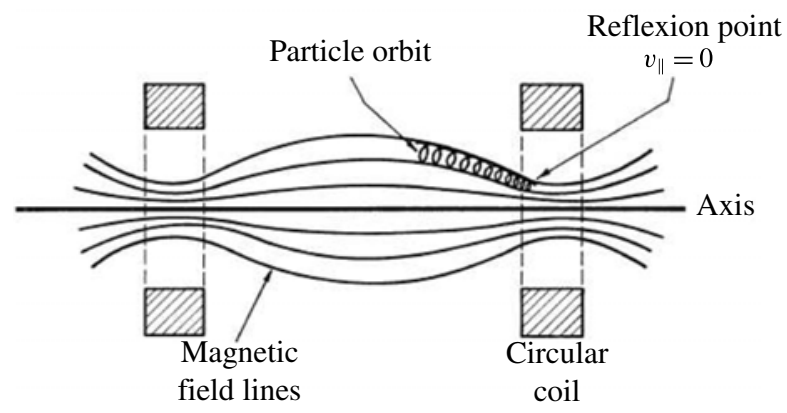

FIGURE 46. Mirror machine (from Taylor 1968).

An adiabatic invariant arises when the motion of a classical particle, with Hamiltonian $H\left(p, q, a_{i}\right)$, is periodic - as it must be in one dimension if it is bounded (Goldstein 1957). Then if the parameters $a_{i}$ are changed slowly compared to the period of oscillation, the resulting quasi-periodic motion possesses an invariant

$$
I=\oint p \mathrm{~d} q,
$$

which acts as a 'constant of motion' in a sense that will be explained shortly.

The classic example of such an invariant is associated with the motion of a pendulum whose length is changed slowly. In this case the invariant is the energy $E(t)$ divided by the frequency $\omega(t)$ of the pendulum,

$$
I \equiv \frac{E}{\omega},
$$

i.e. as the length of the pendulum is altered, the change in its energy is proportional to the change in its frequency. (This is a hint that there might be a connection with quantum states, $\left.\{E=I \omega\}_{\text {classical }} \rightarrow\{E=(n+1 / 2) \hbar \omega\}_{\text {quantum }}.\right)$

It is important to note that the distinguishing feature of an invariant is not, as is sometimes stated, that it varies slowly - it is that changes in the invariant remain small for an indefinite time.

Now let us look at what we mean when we say that the invariant acts as a constant of motion.

The Hamiltonian for a harmonic oscillator with unit mass is

$$
H=\frac{p^{2}}{2}+\omega^{2}(t) \frac{q^{2}}{2} \equiv E
$$

and, when $\omega$ is constant,

$$
p=a \cos (\omega t+\lambda), \quad q=a \omega \sin (\omega t+\lambda) .
$$

To find the invariant when $\omega(t)$ varies slowly, we first transform to new variables $P$ and $Q$, such that

$$
\begin{aligned}
& p \rightarrow(2 \omega P)^{1 / 2} \cos Q \\
& q \rightarrow\left(\frac{2 P}{\omega}\right)^{1 / 2} \sin Q
\end{aligned}
$$


(The generating function for this transformation is $F(q, Q)=(\omega / 2) q^{2}$ cot $Q$; see Goldstein (1957).) Then the new Hamiltonian is

$$
H=\omega(t) P+\frac{\dot{\omega}}{2 \omega} P \sin 2 Q,
$$

and the equations of motion become

$$
\begin{aligned}
\dot{P} & =-\frac{\dot{\omega}}{\omega} P \cos 2 Q, \\
\dot{Q} & =\omega+\frac{\dot{\omega}}{2 \omega} \sin 2 Q .
\end{aligned}
$$

We now introduce a slow time dependence of the oscillation frequency, via the small parameter $\epsilon$, by writing $\omega=\omega(\epsilon t)$, and look for an invariant as a power series in $\epsilon$ :

$$
\text { constant }=J(P, Q, \epsilon t)=J_{0}(P, Q)+\epsilon J_{1}(P, Q, \epsilon t)+\epsilon^{2} J_{2}(P, Q, \epsilon t)+\cdots .
$$

Then requiring

$$
\frac{\mathrm{d} J}{\mathrm{~d} t}=\epsilon \frac{\partial J}{\partial t}+\frac{\partial J}{\partial P} \dot{P}+\frac{\partial J}{\partial Q} \dot{Q}=0
$$

and using (16.11), we obtain, order by order, the recurrence relation

$$
\omega \frac{\partial J_{n}}{\partial Q}=-\frac{\partial J_{n-1}}{\partial t}-\frac{\dot{\omega}}{\omega}\left[\frac{1}{2} \sin 2 Q \frac{\partial J_{n-1}}{\partial Q}-P \cos 2 Q \frac{\partial J_{n-1}}{\partial P}\right] .
$$

This allows us to generate the invariant term by term. The first term $J_{0}$ must be a function of $P$ only, and if we choose $J_{0} \equiv P$ then, using (16.8), we can confirm that this is indeed the integral $I$ introduced earlier (equation (16.3)),

$$
I=\oint p \mathrm{~d} q=\oint \sqrt{2 \omega P} \cos Q \sqrt{\frac{2 P}{\omega}} \cos Q \mathrm{~d} Q \equiv P .
$$

The next few terms in the series are

$$
\begin{gathered}
J_{1}=\frac{1}{2}\left(\frac{\dot{\omega}}{\omega^{2}}\right) P \sin 2 Q, \\
J_{2}=\frac{1}{8}\left(\frac{\dot{\omega}}{\omega^{2}}\right)^{2} P+\frac{1}{4 \omega} \frac{\partial}{\partial t}\left(\frac{\dot{\omega}}{\omega^{2}}\right) P \cos 2 Q, \\
J_{3}=\left[\frac{1}{16}\left(\frac{\dot{\omega}}{\omega^{2}}\right)^{3}-\frac{1}{8}\left(\frac{1}{\omega} \frac{\partial}{\partial t}\right)^{2}\left(\frac{\dot{\omega}}{\omega^{2}}\right)\right] P \sin 2 Q,
\end{gathered}
$$

and this pattern is followed by subsequent terms: odd-numbered terms $\sim \sin 2 Q$ and even-numbered terms $\sim \cos 2 Q$ plus a quantity independent of $Q$.

So, now we see that $I=P=\oint p \mathrm{~d} q$ is actually only the first term of an expansion of the invariant $J$ in powers of $\epsilon$. However, the important point is that all the higher-order terms in this series are small and vanish when $\omega(t)$ is constant. So if $\omega(t)$ starts from a steady value at $t \rightarrow-\infty$, then changes slowly before eventually returning to a steady 
value at $t \rightarrow+\infty$, the initial and final values of $I=\oint p \mathrm{~d} q$ should be the same. Hence, in this sense the series (16.12) is an exact constant of motion.

Perhaps surprisingly, this is not what actually happens. In fact, the initial and final values of $I$ differ by a small quantity that does not appear in the power series because it cannot be expanded in $\epsilon$. This difference, often called the 'non-adiabatic change', is of the form (Hastie et al. 1969)

$$
\frac{\Delta I_{n a}}{I} \sim A \mathrm{e}^{-\alpha / \epsilon} \cos \phi,
$$

where $A$ and $\alpha$ are constants as $\epsilon \rightarrow 0$ and $\phi$ is the phase of the oscillation at some reference point (usually the point at which the phase is changing most slowly). This situation leads to the statement that an adiabatic invariant is 'constant to all orders but not a constant'.

(A hint that there must be a non-adiabatic contribution comes from the correspondence between a classical invariant and quantum states mentioned earlier. If the frequency of a quantum oscillator is altered slowly and then returned to its original value, the oscillator would not return to its original state. Instead, it would have an exponentially small probability of being in 'excited' states. The classical limit of this is the non-adiabatic change.)

A rigorous calculation of the non-adiabatic change (which, of course, cannot be obtained from an expansion or iteration in $\epsilon$ ) is very lengthy, but a strong indication of its exponential form can be given rather easily. If we return to (16.11),

$$
\begin{aligned}
\dot{P} & =-\frac{\dot{\omega}}{\omega} P \cos 2 Q, \\
\dot{Q} & =\omega+\frac{\dot{\omega}}{2 \omega} \sin 2 Q,
\end{aligned}
$$

and in the equation for $\dot{P}$ simply replace $Q$ by its first approximation,

$$
Q \sim \int^{t} \omega\left(\epsilon t^{\prime}\right) \mathrm{d} t^{\prime}
$$

we have

$$
\frac{\Delta P}{P} \sim \int_{-\infty}^{\infty} \frac{\dot{\omega}(\tau)}{\omega(\tau)} \exp \left[-\frac{\mathrm{i}}{\epsilon} \int^{\tau} \omega\left(\tau^{\prime}\right) \mathrm{d} \tau^{\prime}\right] \mathrm{d} \tau .
$$

If $\omega(\tau)$ is analytic, this can be evaluated by contour integration, with the result

$$
\frac{|\Delta P|}{P}=A \exp \left(-\frac{\alpha}{\epsilon}\right),
$$

where $A$ and $\alpha$ are related to the order and position of the (complex) zero of $\omega(\tau)$ closest to the real axis.

\subsection{Invariants and particle orbits}

The motion of a charged particle in a time-dependent uniform field $B(t)$ is exactly equivalent to a harmonic oscillator. To see this, it is convenient to take the vector potential

$$
\boldsymbol{A}(t)=(-y B(t), x B(t), 0)
$$


Then the Hamiltonian is

$$
H=\frac{1}{2}\left(p_{x}+y B(t)\right)^{2}+\frac{1}{2}\left(p_{y}-x B(t)\right)^{2},
$$

from which we get the equations of motion in the form

$$
\ddot{z}+\mathrm{i} B \dot{z}+\mathrm{i} \frac{\dot{B}}{2} z=0
$$

where $z=x+\mathrm{i} y$. (The term in $\dot{z}$ is the Lorentz, $\boldsymbol{v} \times \boldsymbol{B}$, term and that in $\dot{B}$ is due to the induced electric field.) Writing

$$
z=q \exp \left(-\mathrm{i} \int^{t} \frac{B(\tau)}{2} \mathrm{~d} \tau\right)
$$

reduces (16.27) to the oscillator equation

$$
\ddot{q}+\frac{1}{4} B^{2}(t) q=0 .
$$

So we can carry over the results we obtained for the oscillator to the charged particle in a uniform field: then the lowest-order invariant is the magnetic moment $m v_{\perp}^{2} / B$ as we anticipated.

Unfortunately, we are interested in the motion of a particle in a static, non-uniform field, rather than in a time-dependent, uniform one. This is a far more complex problem that cannot be reduced to harmonic motion. Nevertheless, the qualitative features of the adiabatic invariant are similar to those for the harmonic oscillator. Thus there is a power-series expansion for the invariant, now in powers of the ratio of Larmor radius to the scale of the variation in magnetic field. Since this is proportional to $m / e$, we can formally use $\lambda \equiv m / e$ as an expansion parameter; then the first term is $m v_{\perp}^{2} / 2 B$ and there is a non-adiabatic contribution of the form $A \exp (-\alpha / \epsilon) \cos \phi$.

A convenient starting point for calculating the power series is the Vlasov equation for a distribution of particles (see $\S 1.3$ ). (Its connection with the invariants is, of course, that if $I_{j}$ are invariants, then $f\left(I_{j}\right)$ is a stationary distribution.)

Introducing the gyro-phase angle $\phi$ about the magnetic field, the energy $\epsilon$ and the magnetic moment $\mu$ as velocity coordinates, and $(\alpha, \beta, s)$ as spatial coordinates $(\alpha$ and $\beta$ label a field line as in $\S 2.1$ and $s$ is the distance along it), the Vlasov equation

$$
\frac{\partial f}{\partial t}+\boldsymbol{v} \cdot \nabla f+\frac{e_{s}}{m_{s}}(\boldsymbol{v} \times \boldsymbol{B}) \cdot \frac{\partial f}{\partial \boldsymbol{v}}=0
$$

can be written as

$$
\frac{\partial f}{\partial \phi}=\lambda\left(D f+\frac{1}{B} \frac{\partial f}{\partial t}\right) .
$$

Here $D$ is a partial differential operator in $(\phi, \mu, \alpha, \beta, s)$, with coefficients depending on the gradients, curvature and torsion of the field lines.

We now seek a solution of (16.31),

$$
f=f_{0}+\lambda f_{1}+\lambda^{2} f_{2}+\cdots,
$$

that is stationary on ever increasing time scales, $t, t / \lambda, t / \lambda^{2}, \ldots$ It turns out (as detailed in Hastie et al. 1967) that a distribution is stationary on all scales if it 
depends only on three variables - the energy $\epsilon$, the magnetic moment $\mu_{0}=m v_{\perp}^{2} / 2 B$, and a longitudinal invariant

$$
J_{0}=\oint v_{\|} \mathrm{d} s=\oint\left(\epsilon-\mu_{0} B\right)^{1 / 2} \mathrm{~d} s .
$$

The latter is associated with periodic motion along the field (between magnetic mirrors for trapped particles or around closed field lines for passing particles in a torus).

If we now write the true invariants $\hat{\mu}$ and $\hat{J}$ as

$$
\begin{gathered}
\hat{\mu}=\mu_{0}+\lambda \mu_{1}+\lambda^{2} \mu_{2}+\cdots, \\
\hat{J}=J_{0}+\lambda J_{1}+\lambda^{2} J_{2}+\cdots,
\end{gathered}
$$

and identify the terms in the series calculated for a stationary distribution with the corresponding terms of the expansion

$$
f\left(\mu_{0}, J_{0}, \epsilon\right)+\lambda\left(\mu_{1} \frac{\partial f}{\partial \mu_{0}}+J_{1} \frac{\partial f}{\partial J_{0}}\right)+\lambda^{2}+\cdots,
$$

we obtain a recursion equation for the higher-order terms in the invariants $\hat{\mu}$ and $\hat{J}$ :

$$
\left\{\begin{array}{l}
J_{n+1} \\
\mu_{n+1}
\end{array}\right\}=\int D\left\{\begin{array}{l}
J_{n} \\
\mu_{n}
\end{array}\right\}-\sigma \int_{s_{0}}^{s} \frac{B \mathrm{~d} s}{q} L\left\{\begin{array}{l}
J_{n} \\
\mu_{n}
\end{array}\right\}+\int \frac{\mathrm{d} \alpha}{\partial J / \partial \alpha} H\left\{\begin{array}{l}
J_{n} \\
\mu_{n}
\end{array}\right\} .
$$

Here $D$ is the Vlasov operator (16.31), $L$ and $H$ are iterates of it, and $\sigma= \pm 1$ distinguishes the two possible directions of $v_{\|}$. The first few terms, $\mu_{1}, \mu_{2}$ and $J_{1}$, of the invariants are given in Hastie et al. (1967). An unexpected feature is that they depend on the direction $\sigma$ of the particle motion along the magnetic field.

It is not clear that a rigorous calculation of the non-adiabatic contribution to $\mu$ exists for particles in a spatially varying field. Some calculations of it essentially replace the motion in a spatially varying field $B(\boldsymbol{r})$ by motion in a fictitious time-dependent field $B^{*}(t)$ - taken to be the field at the guiding centre of the particle, i.e. $\mathrm{d} B^{*} / \mathrm{d} t=v_{\|} \partial B / \partial s$. This, of course, leads to the exponential form (16.24) dependent on a complex zero of $B^{*}(t)$. (The uncertainty about such a calculation lies in the fact that we are trying to calculate an exponentially small quantity when the guiding centre orbit is only accurate to order $\epsilon$, a larger quantity. Moreover, whereas a particle in a slowly timevarying field experiences only the slow variation in $B(t)$, a particle in a spatially varying field also experiences a 'fast' variation around the Larmor orbit.)

\subsection{Orbit calculations}

The magnetic moment is clearly the most important invariant for plasma confinement in mirrors, and it is interesting therefore to see its behaviour in particle orbits computed in a non-uniform field. This is illustrated in figure 48(a-c), reproduced from Hastie et al. (1969). (Actually, these are for orbits in a quadrupole field, as shown in figure 47 , whose strength is given by

$$
B=\frac{2 I}{a q}\left(1+2 q \cos \Phi+q^{2}\right)^{1 / 4}
$$

so that $\Phi=\pi$ is the weak-field plane of symmetry, $2 a$ is the separation between the current filaments and $q$ labels field lines. In such a field there are both trapped orbits, near the weak-field plane, and untrapped circulating orbits.) 


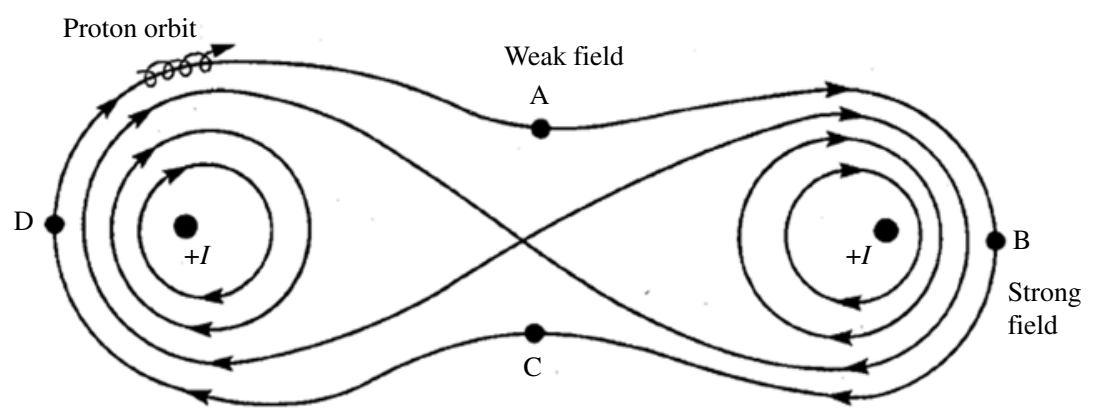

FIGURE 47. Quadrupole field configuration (from Hastie 1993).

Figure 48(a) shows the change in the magnetic moment invariant, calculated from the approximations $\mu_{0}, \quad\left(\mu_{0}+\lambda \mu_{1}\right)$ and $\left(\mu_{0}+\lambda \mu_{1}+\lambda^{2} \mu_{2}\right)$ to the infinite power series (16.34). This orbit starts at the weak-field plane and makes two reflections and two transits of the weak-field plane. The lowest approximation $\mu_{0}=m v_{\perp}^{2} / 2 B$ shows significant oscillations between transits and larger 'jumps' as it crosses the weak-field plane. As higher-order terms are added to the power series, the oscillations disappear, but the 'jumps' remain.

The exponential form of these non-adiabatic jumps is confirmed by figure $48(b)$. (More extensive calculations of these jumps can be found in Howard (1971).)

Figure 48(c) shows the non-adiabatic changes over many transits of the weak-field plane. Owing to the large number of Larmor cycles that occur between successive transits, the phase at each transit is effectively random. After several transits, the particle is scattered from a trapped orbit to a passing one and back again (the boundary between trapped and passing orbits is shown by the dashed line).

It should be clearly understood that the parameters for these calculations were chosen to illustrate the points of interest and are not representative of typical mirror machines.

Finally, we should note that there is actually another invariant, in addition to $\hat{\mu}$ and $\hat{J}$, for a particle in a mirror machine, associated with the periodic orbit of the guiding centre as it drifts around the axis of symmetry. The invariant in this case is the magnetic flux enclosed by the drift orbit. However, it is seldom invoked, as the drift motion can be studied directly (see $\S 20$ ).

\subsection{Summary}

Adiabatic invariants are subtle quantities, and in the case of charged particles in a nonuniform magnetic field they are complicated functions of the field gradient, curvature and torsion. However, in practice, provided the Larmor radius is everywhere much smaller than the local field gradient $R=(\nabla B / B)^{-1}$, the magnetic moment is effectively constant - apart from the effect of collisions.

\section{Simple mirror machines}

On the basis of the guiding centre drifts (see $\S 1.3$ ) and the invariance of the magnetic moment, an axisymmetric mirror machine, such as that shown in figure 46, should confine a low- $\beta$ plasma ( $\beta=$ plasma pressure/magnetic pressure). The drifts are azimuthal and cause no direct loss, the mirrors confine particles (except for those 

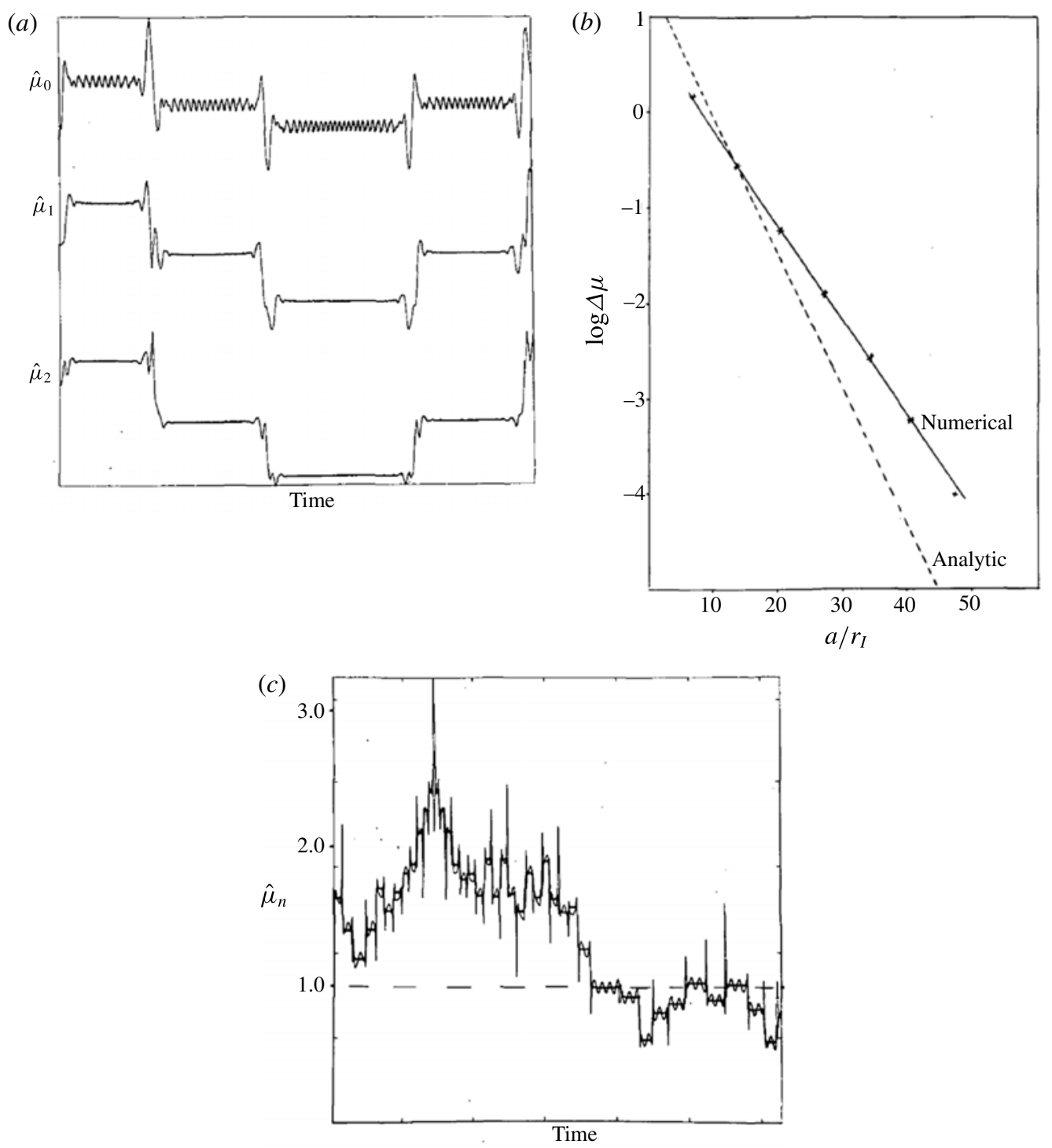

FIGURE 48. (a) Evolution of successive approximations to the particle magnetic moment as it moves in a mirror field. (b) Exponential form of the non-adiabatic jump at each transit as a function of $a / r_{L} \propto 1 / v$. (c) Long-time behaviour of a non-adiabatic particle. Here $q=1.19, \Phi=\pi$ and $r / a=0.075$. (Reproduced with permission from Hastie et al. (1969).)

within the loss cone) and the magnetic field is created solely by external coils and is therefore unconditionally stable!

Unfortunately, there are two serious problems with this simple picture. The first is that collisions cause particles to be scattered into the loss cone. This is a more complex problem than it may appear at first sight (Post 1983, 1987). Electrons are scattered much faster than ions; therefore an electrostatic potential builds up, which narrows the loss cone for electrons (in fact they are essentially confined 

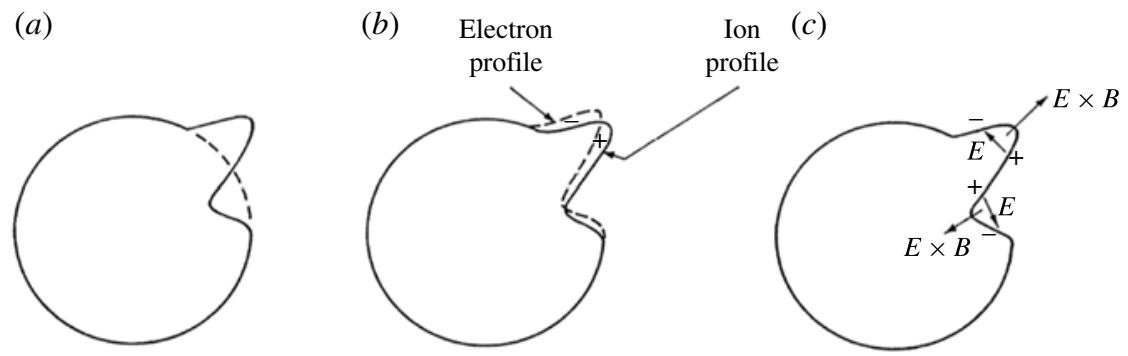

FIGURE 49. Development of the flute instability in a mirror machine (from Taylor 1968); see text for details.

electrostatically) and widens it for ions. Also, the loss cone varies with the local field strength and therefore with the shape of the field - so the loss does not depend solely on the mirror ratio, $R=B_{\max } / B_{\min }$. Nevertheless, as the result of several computations, it appears that the confinement time set by scattering is a few ion-ion collision times $\tau_{i i}$, and varies very slowly with the mirror ratio. A popular fit to the computations is

$$
\tau_{c} \sim \tau_{i i} \log _{10} R .
$$

In contrast, the confinement in toroidal systems can be many collision times, $\tau_{c} \sim$ $\tau_{i i}\left(a^{2} / \rho_{i}^{2}\right)$.

The second serious problem for mirror machines is that the system is unstable even at low plasma pressure. This instability arises in the following way (see figure 49). At low $\beta$ the plasma does not have enough energy to distort the near-vacuum magnetic field. Consequently, any perturbation must take the form of a flute-like ridge, aligned with the magnetic field (as shown in cross-section in figure 49a). Then the azimuthal $\operatorname{grad} B$ drifts of ions and electrons, in opposite directions, produce a space charge along the sides of the ridge. This in turn produces an electric field, such that the ensuing $E \times B$ drift increases the amplitude of the deformation. The direction of the drifts is set by the fact that the magnetic field in a simple mirror, since it is essentially a vacuum field, always decreases with distance from the axis, at least in the centre of the machine.

This simple picture already suggests a cure for the instability. If the radial field gradient were reversed, the grad $B$ drift, and therefore the $E \times B$ drift, would reverse, and the growth of any ridge-like deformation would be suppressed rather than amplified.

The recipe for stability in a mirror machine is therefore that the magnetic field should increase in the radial as well as in the axial direction. In fact, we shall investigate fields that increase in all directions from a non-zero minimum. Such fields are now known as minimum- $B$ fields or magnetic wells. (Note that, although the field in an axisymmetric 'spindle' cusp increases in all directions from its centre (see figure 50), it is not classed as a 'minimum- $B$ ' field, as its minimum is zero. Consequently, the criterion for adiabatic invariance of the magnetic moment cannot be satisfied there.)

The problem with this prescription for stability is that one cannot create an axisymmetric vacuum field with the minimum- $B$ property. Axisymmetry is desirable because the $\operatorname{grad} B$ drifts then obviously close and do not lead to particle loss. However, this simplification must be abandoned. 


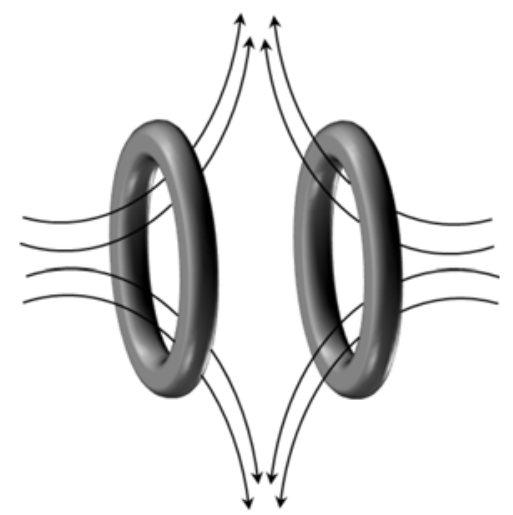

FIGURE 50. Sketch of spindle cusp configuration.

The simplest, non-axisymmetric minimum- $B$ field is created by adding 'Ioffe bars' to the simple mirrors (Gott et al. 1962), as shown in figure 51.

Near the axis the field due to the bars is

$$
\begin{gathered}
B_{r}=-\frac{4 I}{R}\left(\frac{r}{R}\right) \cos 2 \theta, \\
B_{\theta}=\frac{4 I}{R}\left(\frac{r}{R}\right) \sin 2 \theta,
\end{gathered}
$$

where $R$ is the distance of the rods from the axis and $I$ is the current in each rod. If the original axisymmetric mirror field is represented by

$$
\begin{gathered}
B_{z}=B_{0}\left[1-\alpha \mathrm{I}_{0}\left(\frac{2 \pi r}{L}\right) \cos \left(\frac{2 \pi z}{L}\right)\right], \\
B_{\theta}=-\alpha B_{0} \mathrm{I}_{1}\left(\frac{2 \pi r}{L}\right) \sin \left(\frac{2 \pi z}{L}\right),
\end{gathered}
$$

where $\mathrm{I}_{0}$ and $\mathrm{I}_{1}$ are modified Bessel functions, the mirrors are at $z= \pm L / 2$, the mirror ratio $R=(1+\alpha) /(1-\alpha)$, and the field strength near the axis is

$$
B^{2}=B_{0}^{2}(1-\alpha)^{2}+4 \pi^{2} B_{0}^{2}\left\{\alpha(1-\alpha) \frac{z^{2}}{L^{2}}+\frac{r^{2}}{L^{2}}\left[\frac{4 I^{2} L^{2}}{\pi^{2} B_{0}^{2} R^{4}}-\frac{\alpha(1-\alpha)}{2}\right]\right\} .
$$

As the current in the bars is increased, when

$$
I^{2}>\frac{\pi^{2} R^{4}}{8 L^{2}} \alpha(1-\alpha) B_{0}^{2}
$$

the 'magnetic isobars', on which $|\boldsymbol{B}|=$ constant, change from open hyperboloids to closed ellipsoids - the classic minimum- $B$ configuration. This change in the isobars is shown in figure 52 .

A similar minimum- $B$ configuration can be formed from a single coil - the 'tennis ball seam' (see figure 53). (This was invented at Culham and independently at Livermore - where of course it was dubbed the 'baseball seam'!) 
(a)

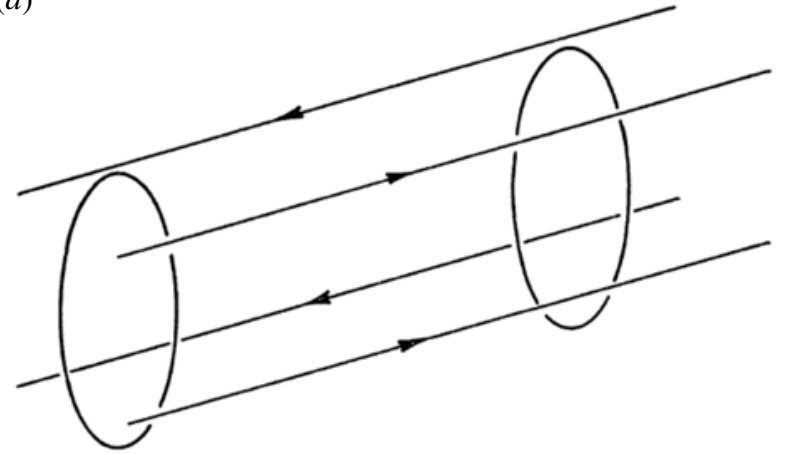

(b)

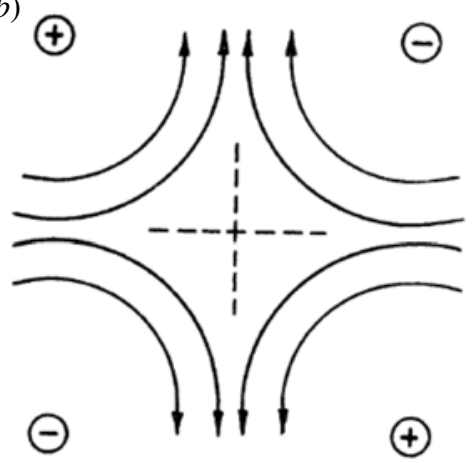

FIgURE 51. (a) Mirror machine stabilisation by Ioffe bars and $(b)$ the resulting magnetic field (from Taylor 1968).

(a)

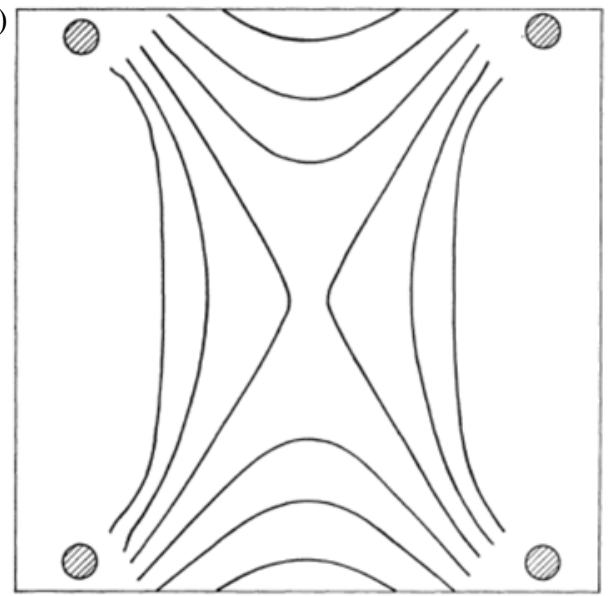

$(b)$

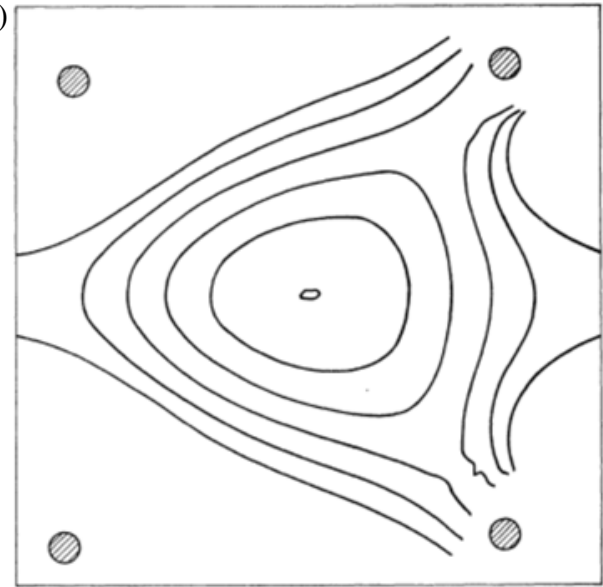

FIgURE 52. Magnetic isobars in a stabilised minimum- $B$ mirror device for $(a)$ small current in the stabilising rods and $(b)$ large current in the rods (from Taylor 1968).

A fundamental problem with the field created by the Ioffe bars or the tennis ball coil is the complicated particle drift orbits - it is far from clear that the orbits are closed or that confinement and charge neutrality are maintained.

An important step therefore is the realisation (Taylor 1963, 1965) that containment and stability in magnetic wells can be described solely in terms of their basic property: that the magnetic isobars form a set of closed, nested surfaces surrounding a non-zero minimum of $|\boldsymbol{B}|$. (Note that the magnetic isobars are not flux surfaces. In general, a line of force will cut a magnetic isobar twice, or not at all.)

\section{Equilibria in magnetic wells}

\subsection{Fluid picture}

For equilibrium in minimum- $B$ systems, the pressure tensor,

$$
\boldsymbol{P}=p_{\perp} \boldsymbol{I}+\left(p_{\|}-p_{\perp}\right) \boldsymbol{n n},
$$




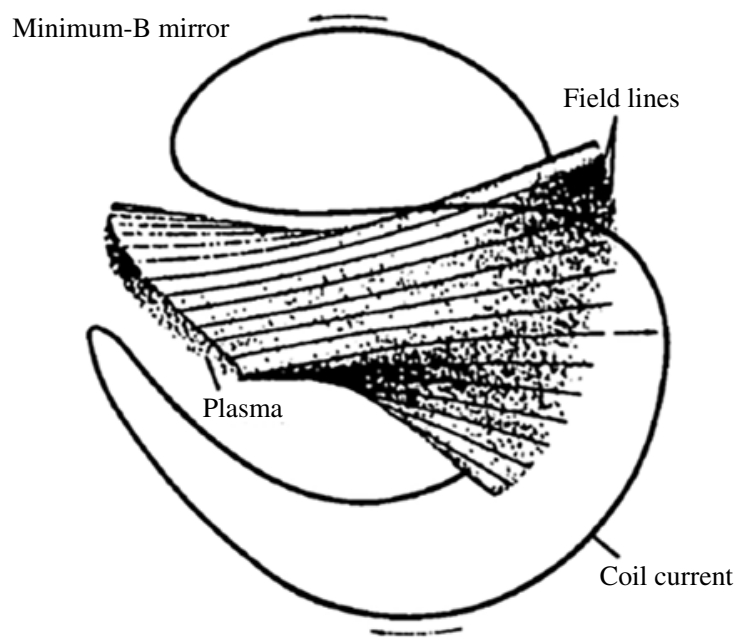

FIGURE 53. Schematic of the tennis ball coil and field lines. (Reproduced with permission from Post (1987).)

which is diagonal but not isotropic, must satisfy (see $§ 1.3$ )

$$
j \times B=\nabla \cdot P,
$$

where $\boldsymbol{n}$ is a unit vector in the direction of $\boldsymbol{B}$ and $\boldsymbol{I}$ is the unit tensor. From the parallel component of this, we find a condition on the pressure,

$$
\frac{\partial p_{\|}}{\partial s}+\frac{\left(p_{\perp}-p_{\|}\right)}{B} \frac{\partial B}{\partial s}=0,
$$

where $s$ is distance along the field.

If (18.3) is satisfied, we can solve (18.2) for $\boldsymbol{j}_{\perp}$,

$$
\boldsymbol{j}_{\perp}=-\frac{\nabla p_{\perp} \times \boldsymbol{B}}{B^{2}}+\frac{\boldsymbol{B} \times \nabla \cdot\left[\left(p_{\|}-p_{\perp}\right) \boldsymbol{n n}\right]}{B^{2}} .
$$

Charge neutrality then imposes a restriction on $\boldsymbol{j}_{\|}=\lambda \boldsymbol{B}$, as in $\S 2$,

$$
\nabla \cdot \boldsymbol{j}_{\|}=\boldsymbol{B} \cdot \nabla \lambda=-\nabla \cdot \boldsymbol{j}_{\perp}
$$

which after some manipulation leads to

$$
\boldsymbol{B} \cdot \nabla \lambda=-\nabla\left(p_{\perp}+p_{\|}\right) \cdot \frac{\boldsymbol{B} \times \nabla B}{B^{3}} .
$$

Integrating this from the point at which a line of force enters the plasma to the point at which it leaves thus gives the constraint

$$
\int \nabla\left(p_{\perp}+p_{\|}\right) \cdot \frac{\boldsymbol{B} \times \nabla B}{B^{4}} \mathrm{~d} s=0 .
$$

If this were not satisfied, there would be currents in the plasma-free region or accumulations of charge in the plasma region.

The constraint (18.7) is automatically satisfied in an axisymmetric system, but in non-axisymmetric systems it is a powerful restriction. 


\subsection{Particle picture}

If $f(\mu, \epsilon, \alpha, \beta)$ is the distribution of guiding centres on a field line $\alpha, \beta$, then the local density along that line is

$$
\int f(\mu, \epsilon, \alpha, \beta) \frac{B}{(\epsilon-\mu B)^{1 / 2}} \mathrm{~d} \mu \mathrm{d} \epsilon,
$$

and the pressure tensor is therefore

$$
\begin{aligned}
& p_{\perp}=\int \mathrm{d} \mu \mathrm{d} \epsilon f(\mu, \epsilon, \alpha, \beta) \frac{\mu B^{2}}{2(\epsilon-\mu B)^{1 / 2}}, \\
& p_{\|}=\int \mathrm{d} \mu \mathrm{d} \epsilon f(\mu, \epsilon, \alpha, \beta) B(\epsilon-\mu B)^{1 / 2} .
\end{aligned}
$$

These expressions automatically satisfy the parallel equilibrium constraint (18.3).

The second constraint (18.7), which restricts the function $f(\mu, \epsilon, \alpha, \beta)$, can be reinterpreted in terms of particle drifts as

$$
\int\left(\nabla \cdot j_{D}\right) \frac{\mathrm{d} l}{B}=0
$$

where the diamagnetic current $\boldsymbol{j}_{D}$ is given by the sum over species of the grad $B$ drift velocities,

$$
\boldsymbol{v}_{B}=-\frac{m_{s}}{e_{s}} \frac{\nabla B \times \boldsymbol{B}}{B^{3}}(2 \epsilon-\mu B) .
$$

This current due to guiding centre drifts is not the total current - there is also a current due to the gradient in density of guiding centres. However, this can be expressed as the curl of the magnetisation per unit volume and is therefore divergence-free.

\subsection{Special minimum-B equilibria}

A class of equilibria that satisfy (18.7) are those for which $\left(p_{\perp}+p_{\|}\right)$is a function of the field strength $B$ only. Then the parallel constraint (18.3) makes $p_{\perp}$ and $p_{\|}$ separately functions of $B$ only. Thus $p_{\perp}=p_{\perp}(B), p_{\|}=p_{\|}(B)$ and $B p_{\|}^{\prime}=p_{\|}-p_{\perp}$. Either of the two functions, $p_{\perp}(B)$ or $p_{\|}(B)$, can be chosen arbitrarily, but the other is then fixed by the parallel equilibrium constraint. In terms of a particle description of the plasma, these special equilibria are those in which the distribution function $f(\mu, \epsilon, \alpha, \beta)$ is independent of $\alpha, \beta$, that is, $f \equiv f(\mu, \epsilon)$.

The significance of magnetic fields that possess closed magnetic isobars is now apparent. Equilibria in which $p_{\perp}$ and $p_{\|}$are functions of $B$ may exist in many field configurations, but only in those which possess closed magnetic isobars can these equilibria represent confined plasmas. (We will examine their stability in the next section.)

A simple example of this class of equilibria is

$$
\begin{aligned}
& \left.\begin{array}{l}
p_{\|}=c B\left(B_{0}-B\right)^{n} \\
p_{\perp}=n c B^{2}\left(B_{0}-B\right)^{n-1}
\end{array}\right\} \quad \text { for } B<B_{0}, \\
& p_{\perp}=p_{\|}=0 \quad \text { for } B>B_{0} \text {. }
\end{aligned}
$$


These represent plasma confined within the contour $B=B_{0}$, which by construction is closed. The particle distribution corresponding to this example is

$$
\begin{array}{ll}
f(\mu, \epsilon)=g(\mu)\left(\mu B_{0}-\epsilon\right)^{n-3 / 2} & \text { for } \epsilon<\mu B_{0} \\
f(\mu, \epsilon)=0 & \text { for } \epsilon>\mu B_{0} \text { (the loss cone), }
\end{array}
$$

where $g(\mu)$ is an arbitrary function and $n>3 / 2$.

\section{Energy principles and stability}

One of the most useful ways of investigating plasma stability is by means of 'energy principles'. From the mathematical point of view, these principles have some subtle and questionable features, but the underlying concept is straightforward.

Consider first the case of a scalar pressure plasma, with an adiabatic equation of state (see $\S 1.3$, now setting the free-space permeability $\mu_{0} \equiv 1$ for clarity). The equations governing linear perturbations of a static equilibrium are then

$$
\begin{gathered}
\rho \frac{\partial \boldsymbol{v}_{1}}{\partial t}=\boldsymbol{j}_{1} \times \boldsymbol{B}+\boldsymbol{j} \times \boldsymbol{B}_{1}-\nabla p_{1}, \\
\frac{\partial p_{1}}{\partial t}=-\boldsymbol{v}_{1} \cdot \nabla p-\gamma p \nabla \cdot \boldsymbol{v}_{1}, \\
\frac{\partial \boldsymbol{B}_{1}}{\partial t}=\nabla \times\left(\boldsymbol{v}_{1} \times \boldsymbol{B}\right) .
\end{gathered}
$$

Combining these, we can form an equation of motion, with $\tilde{\boldsymbol{Q}}=\nabla \times\left(\boldsymbol{v}_{1} \times \boldsymbol{B}\right)$,

$$
\rho \frac{\partial^{2} \boldsymbol{v}_{1}}{\partial t^{2}}=(\nabla \times \tilde{\boldsymbol{Q}}) \times \boldsymbol{B}+\boldsymbol{j} \times \tilde{\boldsymbol{Q}}+\nabla\left(\boldsymbol{v}_{1} \cdot \nabla p+\gamma p \nabla \cdot \boldsymbol{v}_{1}\right)
$$

If the perturbation takes the form of a normal mode, $\boldsymbol{v}_{1}=\mathrm{i} \omega \boldsymbol{\xi}(\boldsymbol{x}) \exp (-\mathrm{i} \omega t)$, where $\xi$ is the plasma displacement, the equation of motion becomes

$$
-\omega^{2} \rho \boldsymbol{\xi}=\boldsymbol{F}(\boldsymbol{\xi})
$$

It can be shown that $\boldsymbol{F}(\boldsymbol{\xi})$ is self-adjoint (see Bernstein et al. 1958; Freidberg, 1987), and therefore

$$
\omega^{2} \int_{V} \rho|\xi|^{2} \mathrm{~d} \tau=-\int_{V} \boldsymbol{\xi}^{*} \cdot \boldsymbol{F}(\boldsymbol{\xi}) \mathrm{d} \tau=\delta W_{1}\left(\boldsymbol{\xi}^{*}, \boldsymbol{\xi}\right)
$$

where

$$
\delta W_{1}\left(\boldsymbol{\xi}^{*}, \boldsymbol{\xi}\right)=\int_{V} \mathrm{~d} \tau\left\{|\boldsymbol{Q}|^{2}-\boldsymbol{\xi}_{\perp}^{*} \cdot \boldsymbol{j} \times \boldsymbol{Q}+\gamma p|\nabla \cdot \boldsymbol{\xi}|^{2}+\left(\boldsymbol{\xi}_{\perp} \cdot \nabla p\right) \nabla \cdot \boldsymbol{\xi}_{\perp}^{*}\right\}
$$

and

$$
\boldsymbol{Q}=\nabla \times(\xi \times B) .
$$

The functional $\delta W_{1}\left(\xi^{*}, \xi\right)$ is clearly the change in potential energy due to the perturbation $\xi$. If it is positive for all $\xi$, then $\omega$ is real and the plasma is stable against all displacements $\xi$. 
This form of energy principle is the classic one introduced by Bernstein et al. (1958). It will later form the starting point for the extensive analysis of ballooning modes (see §24). But, of course, it is not applicable to mirror machines - since these always involve anisotropic pressure.

An energy principle for plasmas with anisotropic pressure was also given by Bernstein et al. (1958), namely

$$
\begin{aligned}
\delta W_{2}= & \int_{V} \mathrm{~d} \tau\left\{|\boldsymbol{Q}|^{2}-\boldsymbol{j} \cdot \boldsymbol{Q} \times \boldsymbol{\xi}+\frac{5}{3} p_{\perp}(\boldsymbol{\nabla} \cdot \boldsymbol{\xi})^{2}+(\nabla \cdot \boldsymbol{\xi})\left(\boldsymbol{\xi} \cdot \nabla p_{\perp}\right)\right. \\
& +\frac{1}{3} p_{\perp}(\nabla \cdot \boldsymbol{\xi}-3 q)^{2}+q \nabla \cdot\left[\boldsymbol{\xi}\left(p_{\|}-p_{\perp}\right)\right] \\
& \left.-\left(p_{\|}-p_{\perp}\right)\left[\boldsymbol{n} \cdot(\boldsymbol{a} \cdot \boldsymbol{\nabla}) \boldsymbol{\xi}+\boldsymbol{a} \cdot(\boldsymbol{n} \cdot \boldsymbol{\nabla}) \boldsymbol{\xi}-4 q^{2}\right]\right\}
\end{aligned}
$$

where

$$
\begin{gathered}
q=\boldsymbol{n} \cdot(\boldsymbol{n} \cdot \nabla) \xi, \\
\boldsymbol{a}=(\boldsymbol{n} \cdot \nabla) \xi-(\xi \cdot \nabla) \boldsymbol{n} .
\end{gathered}
$$

You might notice that (19.9) does not reduce to the scalar pressure form (19.7) when one sets $p_{\perp}=p_{\|}$. This is because in $\delta W_{1}$ the pressure is isotropic in both the equilibrium and the perturbation, whereas when $p_{\perp}=p_{\|}$in $\delta W_{2}$ the pressure is isotropic in the equilibrium but need not be in the perturbation.

Unfortunately, even $\delta W_{2}$ is not appropriate for plasmas confined by mirrors, as it assumes there is no heat flow along the field. An energy principle that overcomes this limitation was given by Kruskal \& Oberman (1958). This is derived somewhat differently from the principles discussed above. It starts from the plasma energy in the small-Larmor-radius limit,

$$
\mathcal{E}=\int_{V} \frac{1}{2}\left(|\boldsymbol{B}|^{2}+|\boldsymbol{E}|^{2}\right) \mathrm{d} \tau+\sum_{s} \int_{V} \iint\left[\left(\epsilon+\frac{1}{2} v_{E}^{2}\right) m_{s} f(\epsilon, \mu, \boldsymbol{x})\right] \frac{B}{v_{\|}} \mathrm{d} \mu \mathrm{d} \epsilon \mathrm{d} \tau,
$$

(here $\mu=v_{\perp}^{2} / 2 B$ and $v_{\|}=\sqrt{2(\epsilon-\mu B)}$ ). Any perturbation of this energy will contain a part dependent on the perturbed magnetic field and a part dependent on the perturbed particle distribution. However, because the guiding centre drift $v_{E}$ is the $E \times B$ velocity, we can introduce a displacement $\xi$ that links particle motion to changes in the magnetic field (the frozen-in field condition, see $\S 4.1$ ). Then, holding $\xi$ fixed and minimising over the particle distribution gives a form $\delta W_{3}$ that depends (after some algebra; see Kruskal \& Oberman 1958; Taylor \& Hastie 1965) only on the displacement $\xi$ and equilibrium quantities

$$
\begin{aligned}
\delta W_{3}= & \int_{V} \mathrm{~d} \tau\left\{|\boldsymbol{Q}|^{2}-\boldsymbol{j} \cdot \boldsymbol{Q} \times \boldsymbol{\xi}+\left(2 p_{\perp}+C\right)(\boldsymbol{\nabla} \cdot \boldsymbol{\xi}-q)^{2}\right. \\
& +\boldsymbol{\xi} \cdot \nabla p_{\perp}(\boldsymbol{\nabla} \cdot \boldsymbol{\xi}-q)+\left(\boldsymbol{\xi} \cdot \boldsymbol{\nabla} p_{\|}\right) q \\
& -\left(p_{\|}-p_{\perp}\right)\left[\boldsymbol{n} \cdot(\boldsymbol{a} \cdot \boldsymbol{\nabla}) \boldsymbol{\xi}+\boldsymbol{a} \cdot(\boldsymbol{n} \cdot \boldsymbol{\nabla}) \boldsymbol{\xi}-q^{2}-q \boldsymbol{\nabla} \cdot \boldsymbol{\xi}\right] \\
& \left.-\sum_{s} m_{s} \iint \frac{B}{v_{\|}} \frac{\partial f}{\partial \epsilon}\left\langle v_{\|}^{2} q+\mu B(\boldsymbol{\nabla} \cdot \boldsymbol{\xi}-q)\right\rangle^{2} \mathrm{~d} \mu \mathrm{d} \epsilon\right\},
\end{aligned}
$$


where the average in the last term is defined as

$$
\langle g\rangle=\left(\int g \frac{\mathrm{d} l}{v_{\|}}\right)\left(\int \frac{\mathrm{d} l}{v_{\|}}\right)^{-1}
$$

and

$$
C=\sum_{s} m_{s} \iint \frac{B}{v_{\|}} \frac{\partial f}{\partial \epsilon}(\mu B)^{2} \mathrm{~d} \mu \mathrm{d} \epsilon
$$

\subsection{Application to special minimum- $B$ equilibria}

For the special equilibria introduced in $\S 18.3, p_{\|}=p_{\|}(B), p_{\perp}=p_{\perp}(B)$ and

$$
B p_{\|}^{\prime}=p_{\|}-p_{\perp}, \quad B p_{\perp}^{\prime}=2 p_{\perp}+C, \quad j_{\|}=0,
$$

where the vanishing of $j_{\|}$follows from (18.7). A more convenient form of $\delta W_{3}$ is then (Taylor \& Hastie 1965)

$$
\begin{aligned}
\delta W_{3}= & \int_{V} \mathrm{~d} \tau\left\{\left|\boldsymbol{Q}_{\perp}\right|^{2}\left(1+\frac{p_{\perp}-p_{\|}}{B^{2}}\right)+Q_{\|}^{2}\left(1+\frac{2 p_{\perp}+C}{B^{2}}\right)\right. \\
& -j_{\|}\left(\boldsymbol{n} \cdot \boldsymbol{Q} \times \boldsymbol{\xi}_{\perp}\right)\left(1+\frac{p_{\perp}-p_{\|}}{B^{2}}\right)+q\left[\boldsymbol{\xi} \cdot \nabla p_{\|}+\left(p_{\perp}-p_{\|}\right) s\right] \\
& -\left(2 \frac{Q_{\|}}{B}+s\right)\left[\xi \cdot \nabla p_{\perp}-\left(2 p_{\perp}+C\right) s\right] \\
& \left.-\sum_{s} m_{s} \iint \frac{B}{v_{\|}} \frac{\partial f}{\partial \epsilon}\left\langle v_{\|}^{2} q-\mu B\left(\frac{Q_{\|}}{B}+s\right)\right\rangle^{2} \mathrm{~d} \mu \mathrm{d} \epsilon\right\},
\end{aligned}
$$

where $s=\xi \cdot \nabla B / B$. Using (19.16) this reduces to

$$
\begin{aligned}
\delta W_{3}= & \int_{V} \mathrm{~d} \tau\left\{\left|\boldsymbol{Q}_{\perp}\right|^{2}\left(1+\frac{p_{\perp}-p_{\|}}{B^{2}}\right)+Q_{\|}^{2}\left(1+\frac{2 p_{\perp}+C}{B^{2}}\right)\right. \\
& \left.-\sum_{s} m_{s} \iint \frac{B}{v_{\|}} \frac{\partial f}{\partial \epsilon}\left\langle v_{\|}^{2} q-\mu B\left(\frac{Q_{\|}}{B}+s\right)\right\rangle^{2} \mathrm{~d} \mu \mathrm{d} \epsilon\right\} .
\end{aligned}
$$

Therefore, sufficient conditions for stability of the special equilibria are

$$
B^{2}-p_{\|}+p_{\perp}>0, \quad B^{2}+2 p_{\perp}+C>0,
$$

which, using (19.16), can be expressed in terms of the pressure gradients as

$$
B-\frac{\mathrm{d} p_{\|}}{\mathrm{d} B}>0, \quad B+\frac{\mathrm{d} p_{\perp}}{\mathrm{d} B}>0 .
$$

The first of these is usually satisfied for plasma contained in a magnetic well as the pressure falls and $B$ increases towards the boundary, i.e. $\mathrm{d} p_{\|} / \mathrm{d} B<0$. By integrating the second inequality from the 'bottom' of the well to the plasma boundary, which is 
a contour of constant $|\boldsymbol{B}|$, we find that the maximum stable pressure is equal to the 'depth' of the well,

$$
p_{\perp, \max }=\left(B_{b}^{2}-B_{c}^{2}\right) / 2,
$$

where $B_{b}$ is the field on the plasma boundary, and $B_{c}$ is the field at the 'bottom' of the well.

We see therefore that the special equilibria in magnetic wells are not only remarkable, in that they can be described without knowledge of the field geometry, but they also have remarkably simple and robust stability properties.

Before we discuss the stability of general equilibria in magnetic wells, which we hope will inherit some of the desirable features of the special equilibria, it is interesting to note a proof of stability that does not require the elaborate structure of the energy principles - although it is applicable only at low $\beta$.

\subsection{Direct proof of stability for special equilibria at low $\beta$}

As long as the motion is conservative, we know from Liouville's theorem that the density of particles in phase space remains constant as one moves with the particles. If the particles also carry with them a constant quantity $\mu$ (the magnetic moment), then we can construct a constant of motion $G(f, \mu)$ such that (Taylor 1963)

$$
\mathcal{S}=\int G(f, \mu) \mathrm{d}^{3} v \mathrm{~d}^{3} x=\text { constant }
$$

or

$$
\int \frac{B}{(\epsilon-\mu B)^{1 / 2}} G(f, \mu) \mathrm{d} \epsilon \mathrm{d} \mu \mathrm{d}^{3} x=\text { constant. }
$$

Now consider a perturbation of $f$ around its initial value $f_{0}$. Then

$$
\delta \mathcal{S}=0=\int\left[G^{\prime}\left(f_{0}, \mu\right) \delta f+G^{\prime \prime}\left(f_{0}, \mu\right) \frac{(\delta f)^{2}}{2}+\cdots\right] \mathrm{d}^{3} v \mathrm{~d}^{3} x,
$$

where the prime denotes the derivative with respect to $f$.

The special equilibria have the property that $f_{0}$ is a function only of $\mu$ and $\epsilon$, so if $f_{0}(\mu, \epsilon)$ is monotonic in $\epsilon$, we can choose the function $G$ such that,

$$
G^{\prime}\left(f_{0}, \mu\right)=\epsilon, \quad G^{\prime \prime}\left(f_{0}, \mu\right)=\left(\frac{\partial f_{0}}{\partial \epsilon}\right)^{-1},
$$

for all $\mu$. Then when $f$ is small,

$$
\int \epsilon \delta f \mathrm{~d}^{3} v \mathrm{~d}^{3} x \sim-\int \frac{(\delta f)^{2}}{2 \partial f_{0} / \partial \epsilon} \frac{B}{(\epsilon-\mu B)^{1 / 2}} \mathrm{~d} \mu \mathrm{d} \epsilon \mathrm{d}^{3} x .
$$

The left-hand side of this equation is the change in energy of the particles, and the right-hand side is positive definite. Hence any perturbation increases the kinetic energy of the particles. Also, at low $\beta$ any change in magnetic field increases its energy. Consequently, the system is stable. In fact, this suggests that at low $\beta$ the special minimum- $B$ equilibria, irrespective of their geometrical shape, are stable to any perturbation that does not affect the magnetic moment! 


\section{Canonical description of guiding centre motion}

In practice, it would be extremely difficult to produce the special equilibria, so we must now turn to more general equilibria in magnetic wells. For this, it is convenient to introduce a description in terms of the average guiding centre drifts (Taylor 1965, 1968).

We know that the instantaneous guiding centre drift of a particle with charge $q$ (see $\S 1.3$ ) is

$$
\boldsymbol{v}_{B}=\frac{1}{q} \frac{\boldsymbol{B} \times \nabla B}{B^{3}}(2 \epsilon-\mu B),
$$

but in view of the rapid motion along lines of force, the instantaneous drift is less significant than the average drift over a 'bounce' period between mirrors. This average drift can be described in a particularly elegant way.

We first take the field lines themselves as our coordinate system, setting (see $\$ 2.1$ )

$$
\boldsymbol{B}=\nabla \alpha \times \nabla \beta, \quad \chi=\int \boldsymbol{B} \cdot \mathrm{d} \boldsymbol{l},
$$

where $\mathrm{d} \boldsymbol{l}$ follows a field line. Then $(\alpha, \beta, \chi)$ locate any point in space, and in these coordinates the instantaneous drift in (say) the $\alpha$ direction is

$$
\frac{\mathrm{d} \alpha}{\mathrm{d} t}=\boldsymbol{v}_{B} \cdot \nabla \alpha=-\frac{1}{q B}(2 \epsilon-\mu B)\left(\frac{\partial B}{\partial \beta}\right)_{\alpha} .
$$

If we now introduce the energy $K(\alpha, \beta, \mu, J)$ defined by

$$
J=\oint_{\alpha \beta}(K-\mu B)^{1 / 2} \frac{\mathrm{d} \chi}{B},
$$

then by direct differentiation

$$
\left(\frac{\partial K}{\partial \beta}\right)_{\alpha, J, \mu}=\left[\oint \frac{(2 K-\mu B)}{B(K-\mu B)^{1 / 2}} \frac{\mathrm{d} \chi}{B}\right]\left[\oint \frac{1}{(K-\mu B)^{1 / 2}} \frac{\mathrm{d} \chi}{B}\right]^{-1}\left(\frac{\partial B}{\partial \beta}\right)_{\alpha, J, \mu} .
$$

The right-hand side of (20.5) is just the average guiding centre drift in the $\alpha$ direction, so the equation of motion for this average drift is

$$
q\left\langle\frac{\mathrm{d} \alpha}{\mathrm{d} t}\right\rangle=-\left(\frac{\partial K}{\partial \beta}\right)_{\alpha, J, \mu}
$$

and in a similar way

$$
q\left\langle\frac{\mathrm{d} \beta}{\mathrm{d} t}\right\rangle=\left(\frac{\partial K}{\partial \alpha}\right)_{\beta, J, \mu}
$$

These are just the Hamiltonian equations of motion for the average drift, with $\alpha, \beta$ as conjugate coordinates and $K(\alpha, \beta, \mu, J)$ as the Hamiltonian.

This result is no coincidence! A guiding centre moves as if it were a 'particle' that has a charge $q$ and magnetic moment $\mu$. The Lagrangian for such a particle is

$$
\mathcal{L}=\frac{1}{2} m v^{2}+q(\boldsymbol{v} \cdot \boldsymbol{A})-q \phi(\alpha, \beta, \chi)-\mu B(\alpha, \beta, \chi) .
$$


If we take the vector potential as $\boldsymbol{A}=(\alpha \boldsymbol{\nabla} \beta-\beta \nabla \alpha) / 2$, so that $\boldsymbol{v} \cdot \boldsymbol{A}=(\alpha \dot{\beta}-\beta \dot{\alpha}) / 2$, then the guiding centre Lagrangian becomes

$$
\mathcal{L}=\frac{1}{2} m v_{d}^{2}+\frac{m \dot{\chi}^{2}}{2 B^{2}}+\frac{q}{2}(\alpha \dot{\beta}-\beta \dot{\alpha})-q \phi(\alpha, \beta, \chi)-\mu B(\alpha, \beta, \chi),
$$

where $m v_{d}^{2}$ is the energy associated with the slow transverse drift and is negligible compared to the energy of the parallel motion. Neglecting this drift energy,

$$
\mathcal{L}=\frac{m \dot{\chi}^{2}}{2 B^{2}}+\frac{q}{2}(\alpha \dot{\beta}-\beta \dot{\alpha})-q \phi(\alpha, \beta, \chi)-\mu B(\alpha, \beta, \chi),
$$

and we see immediately that $\alpha$ and $\beta$ are indeed conjugate Hamiltonian coordinates,

$$
p_{\beta}=\frac{\partial \mathcal{L}}{\partial \dot{\beta}}=\frac{q \alpha}{2}, \quad p_{\alpha}=\frac{\partial \mathcal{L}}{\partial \dot{\alpha}}=-\frac{q \beta}{2} .
$$

\section{General equilibria in mirror machines}

Now that we have the simple forms (20.6) and (20.7) for the average drift motion, we can immediately describe equilibria in a mirror machine (Taylor 1964). If the number of particles with invariants $(\mu, J)$ in a flux tube of cross-section $\mathrm{d} \alpha \mathrm{d} \beta$ is $F(\alpha, \beta, \mu, J, t)$ then

$$
\frac{\partial F}{\partial t}+\left\langle\frac{\mathrm{d} \alpha}{\mathrm{d} t}\right\rangle \frac{\partial F}{\partial \alpha}+\left\langle\frac{\mathrm{d} \beta}{\mathrm{d} t}\right\rangle \frac{\partial F}{\partial \beta}=0
$$

or, using (20.6) and (20.7),

$$
\frac{\partial F}{\partial t}-\frac{\partial K}{\partial \beta} \frac{\partial F}{\partial \alpha}+\frac{\partial K}{\partial \alpha} \frac{\partial F}{\partial \beta}=0 .
$$

In equilibrium, $\partial F / \partial t=0$, so $F$ must depend on $\alpha$ and $\beta$ only through $K(\alpha, \beta, \mu, J)$, i.e.

$$
F_{e q}(\alpha, \beta, \mu, J)=F\{\mu, J, K(\alpha, \beta, \mu, J)\} .
$$

Of course, this just says that $F$ is a function of the constants of motion. More usefully, it means that in the $(\alpha, \beta)$ coordinate plane, $F$ is constant on the surfaces $K=$ constant.

\subsection{Stability of general equilibria in mirror machines at low $\beta$}

At low $\beta$, the most dangerous form of instability is the interchange of flux tubes, because, if the tubes contain equal flux, there is no increase in magnetic energy as there would be for any other perturbation. (In an interchange all the particles in one flux tube are exchanged with those on a neighbouring flux tube as the result of $E \times B$ motion, e.g. as if a circular cross-section tube rotated so that each semicircular half is replaced by the other.)

Consider the interchange of a flux tube (1) with a neighbouring tube (2) (Taylor 1964). Before interchange, the total energy of the particles is

$$
W_{\text {init }}=\int(F(1) K(1)+F(2) K(2)) \mathrm{d} \mu \mathrm{d} J,
$$

where $K(1)=K\left(\mu, J, \alpha_{1}, \beta_{1}\right)$ and $F(1)=F\left(\mu, J, K\left(\mu, J, \alpha_{1}, \beta_{1}\right)\right)$ etc. 


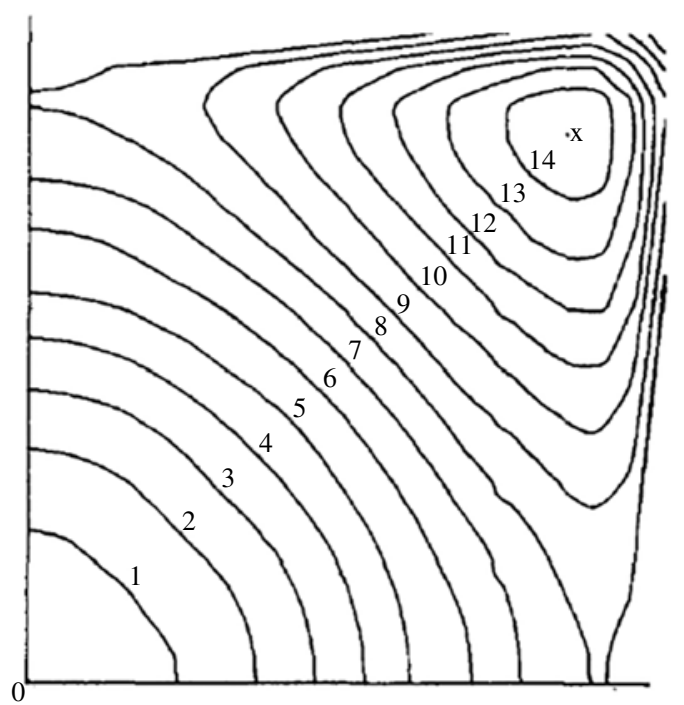

FIGURE 54. Numerical calculation of the constant $K / \mu$ contours in a minimum- $B$ system, for $J / \mu^{1 / 2}=0.305$ (from Taylor 1964).

After interchange, the particles $F(1)$ occupy flux tube (2) and vice versa, so the total energy is then

$$
W_{\text {final }}=\int(F(1) K(2)+F(2) K(1)) \mathrm{d} \mu \mathrm{d} J .
$$

The change in total energy is therefore

$$
\Delta W=-\int \mathrm{d} \mu \mathrm{d} J(F(2)-F(1))(K(2)-K(1)),
$$

and, if the displacements are small,

$$
\delta W=-\int \mathrm{d} \mu \mathrm{d} J\left(\frac{\partial F}{\partial \alpha} \delta \alpha+\frac{\partial F}{\partial \beta} \delta \beta\right)\left(\frac{\partial K}{\partial \alpha} \delta \alpha+\frac{\partial K}{\partial \beta} \delta \beta\right) .
$$

As $F$ depends on $\alpha$ and $\beta$ only through $K$,

$$
\delta W=-\int \mathrm{d} \mu \mathrm{d} J\left(\frac{\partial F}{\partial K}\right)_{\mu, J}\left(\frac{\partial K}{\partial \alpha} \delta \alpha+\frac{\partial K}{\partial \beta} \delta \beta\right)^{2} .
$$

This is clearly positive if

$$
\left(\frac{\partial F}{\partial K}\right)_{\mu, J}<0
$$

for all $\mu, J$ - and this is a sufficient condition for stability. The necessary and sufficient conditions are: $\lambda_{\alpha \alpha}<0, \lambda_{\beta \beta}<0$ and $\left[\lambda_{\alpha \beta}\right]^{2}>\lambda_{\alpha \alpha} \lambda_{\beta \beta}$, where

$$
\lambda_{\alpha \alpha}=\int \mathrm{d} \mu \mathrm{d} J\left(\frac{\partial K}{\partial \alpha}\right)^{2}\left(\frac{\partial F}{\partial K}\right)
$$




$$
\begin{gathered}
\lambda_{\beta \beta}=\int \mathrm{d} \mu \mathrm{d} J\left(\frac{\partial K}{\partial \beta}\right)^{2}\left(\frac{\partial F}{\partial K}\right), \\
\lambda_{\alpha \beta}=\int \mathrm{d} \mu \mathrm{d} J\left(\frac{\partial K}{\partial \alpha}\right)\left(\frac{\partial K}{\partial \beta}\right)\left(\frac{\partial F}{\partial K}\right) .
\end{gathered}
$$

If we want $\partial F / \partial K<0$ and the plasma to be contained (i.e. $F$ decreasing outwards), then the contours of constant $K(\alpha, \beta, \mu, J)$ must form a closed nested set surrounding a minimum, in much the same way that the constant $B$ contours are required to do for the special minimum- $B$ equilibria (see $\S 18.3$ ).

An example of the constant $K$ contours, calculated for an Ioffe-bar-stabilised mirror (see figure 51), is shown in figure 54. The figure shows one quadrant of the cross-section, and the useful confinement region is that inside the last closed contour enclosing the origin. Note that this calculation exploits the fact that $K$ is actually a function of only three variables, since

$$
\frac{J}{\mu^{1 / 2}}=\oint\left[\frac{K}{\mu}-B(\alpha, \beta, \chi)\right]^{1 / 2} \frac{\mathrm{d} \chi}{B},
$$

so that $K=\mu \hat{K}\left(\alpha, \beta, J / \mu^{1 / 2}\right)$.

The constant $K$ contours are analogous to atmospheric isobars. Particles drift along contours of constant $K$ just as the wind flows along isobars, and the drift speed increases as the $K$ contours get closer - just as the wind increases as the isobars get closer.

\section{Maximal ordering and stability}

The discussion of general equilibria above is elegant and, within its limits, complete, but it is unnecessarily detailed for many purposes. Furthermore, as it is restricted to low $\beta$, it cannot determine the all-important maximum stable $\beta$.

To find the maximum $\beta$ for a general equilibrium, we must fall back on an approximate method. This is sometimes called 'maximal ordering' and is a very important technique - not so much for its immediate application but because it is used in many plasma problems, in both mirror and toroidal systems.

To illustrate the method, suppose we start from an energy principle and simply expand $\delta W$ in powers of $\beta$,

$$
\delta W=\delta W_{0}+\beta \delta W_{1}+\beta^{2} \delta W_{2}+\cdots .
$$

If we minimised $\delta W$, we might find $\delta W_{0}^{\min }=0$ and $\delta W_{1}^{\min }>0$. Then we would say that at sufficiently small $\beta$ the plasma is stable, and instability arises only when $\beta^{2} \delta W_{2}^{\min }$ becomes comparable to $\beta \delta W_{1}^{\text {min }}$. If so, we could calculate the critical $\beta$ by evaluating $\delta W_{2}^{\min }$ and setting $\beta \delta W_{1}^{\text {min }}=-\beta^{2} \delta W_{2}^{\text {min }}$. However, this would be unjustified, because the validity of the expansion in $\beta$ is based on the assumption that successive terms get rapidly smaller! (Strictly we should speak about an asymptotic limit as $\beta \rightarrow 0$, and $\beta^{2} \delta W_{2}$ can then never balance $\left.\beta \delta W_{1} !\right)$

To overcome this difficulty, we expand $\delta W$ not just in $\beta$, but in several small quantities, which are then grouped together so that they can balance each other. Usually this is done by allocating to each small quantity a relative order of magnitude in a single small parameter $\lambda$. Then one expands in powers of $\lambda$ and stability is determined by the first non-zero term. Mathematically, this is (hopefully) an 
asymptotic expansion in $\lambda$. Physically, it is based on the fact that, while $\beta$ may not be small, some combination of $\beta$ and other quantities is small.

Of course, the key steps are to select the appropriate group of small quantities and to allocate the appropriate order of magnitude to them. This is something of an art, and it would be difficult to specify an algorithm. However, the next section shows how these key steps are dealt with in order to find the maximum stable $\beta$ in minimum- $B$ mirror machines (Taylor 1965; Taylor \& Hastie 1965).

\subsection{Stability of general equilibria in mirror machines at finite $\beta$}

In order to apply maximal ordering to minimum- $B$ confined plasmas, we regard the zero-order (vacuum) magnetic field as made up of: (i) a uniform field $\boldsymbol{B}_{0}$ in the $z$ direction, (ii) a mirror component $\boldsymbol{B}_{m}$ approximately parallel to $\boldsymbol{B}_{0}$, and (iii) a stabilising component $\boldsymbol{B}_{s}$ approximately perpendicular to $\boldsymbol{B}_{0}$ (as e.g. from Ioffe bars). We also have to include: (iv) the field $\boldsymbol{B}_{\beta}$ produced by the plasma currents and (v) the pressure tensor, with $2 p_{\perp} / B_{0}^{2} \equiv \beta_{\perp}$ and $2 p_{\|} / B_{0}^{2} \equiv \beta_{\|}$. Therefore, for a shallow magnetic well, we already have five small quantities:

$$
\frac{B_{m}}{B_{0}}=\mu, \quad \frac{B_{s}}{B_{0}}=\sigma, \quad \frac{B_{\beta}}{B_{0}}=\beta, \quad \frac{2 p_{\perp}}{B_{0}^{2}}=\beta_{\perp}, \quad \frac{2 p_{\|}}{B_{0}^{2}}=\beta_{\|} .
$$

We want $B_{m}, B_{s}$ and $B_{\beta}$ all to contribute to $|\boldsymbol{B}|^{2}$. However, $B_{s}$ is perpendicular to $B_{0}$, so it contributes only quadratically, as $B_{s}^{2}$, and must therefore be of lower order than $B_{m}$ or $B_{\beta}$, which contribute linearly as $\boldsymbol{B}_{0} \cdot \boldsymbol{B}_{m}$ and $\boldsymbol{B}_{0} \cdot \boldsymbol{B}_{\beta}$. Hence the relative orders must be

$$
\frac{B_{s}}{B_{0}} \sim \lambda, \quad \frac{B_{m}}{B_{0}} \sim \lambda^{2}, \quad \frac{B_{\beta}}{B_{0}} \sim \lambda^{2} .
$$

The relative order of other quantities can be determined from the equilibrium conditions. From the lowest-order equilibrium (see (18.4)),

$$
j_{\perp}^{\beta}=\nabla \times B_{\beta}=\frac{\boldsymbol{B}_{0} \times \nabla p_{\perp}}{B_{0}^{2}}, \quad j_{\|}=0,
$$

we deduce that, since $B_{\beta} / B_{0} \sim \lambda^{2}$, we must take $j_{\perp}^{\beta} \sim \lambda^{2}, p_{\perp} \sim \lambda^{2}$. The order of $p_{\|}$ can similarly be deduced from the parallel equilibrium as $p_{\|} \sim \lambda^{4}$. So now we have a 'maximal ordering',

$$
\begin{gathered}
\quad \frac{B_{s}}{B_{0}} \sim \lambda, \quad \frac{B_{m}}{B_{0}} \sim \lambda^{2}, \quad \frac{B_{\beta}}{B_{0}} \sim \lambda^{2}, \\
j_{\perp} \sim \lambda^{2}, \quad j_{\|} \sim \lambda^{4}, \quad p_{\perp} \sim \lambda^{2}, \quad p_{\|} \sim \lambda^{4} .
\end{gathered}
$$

However, there are two more steps before the algebra can begin! The displacement, $\xi$, that minimises $\delta W(\xi, \xi)$ will itself depend on $\lambda$, so it too must be expanded,

$$
\xi=\xi_{0}+\lambda \xi_{1}+\lambda^{2} \xi_{2}+\cdots,
$$

and, before we can expand the Kruskal-Oberman energy functional (19.17), we must extend the ordering to include terms depending on the distribution function, rather than the displacement $\xi$. From (18.9), the derivative of $p_{\perp}$ along the field gives

$$
\frac{\partial p_{\perp}}{\partial s}=\left(C+2 p_{\perp}\right) \frac{1}{B} \frac{\partial B}{\partial s},
$$


where $C$ was given in (19.15). As we have already found both $\partial p_{\perp} / \partial s$ and $\partial B / \partial s$ to be of order $\lambda^{2}$, we must treat $C$ as a zero-order quantity.

So now, at last, we can expand $\delta W(\xi, \xi)$ in powers of $\lambda$. It will be necessary to go to fourth order, $\lambda^{4}$, and this involves a lot of algebra.

In zeroth order, we find

$$
\delta W_{0}=\int_{V} \mathrm{~d} \tau\left\{\left[\boldsymbol{Q}_{\perp 0}\right]^{2}+\left(1+\frac{C}{B_{0}^{2}}\right)\left[Q_{\| 0}\right]^{2}-\sum_{s} m_{s} \iint \frac{B_{0}}{v_{\|}} \frac{\partial f}{\partial \epsilon} \mu^{2}\left\langle Q_{\| 0}\right\rangle^{2} \mathrm{~d} \mu \mathrm{d} \epsilon\right\} .
$$

We will assume that $\partial f / \partial \epsilon<0$ throughout, so $\delta W_{0}$ is certainly positive when

$$
\left(1+C / B_{0}^{2}\right)>0 .
$$

However, the minimum $\delta W_{0}$ is zero when $\boldsymbol{Q}_{0}=\nabla \times\left(\boldsymbol{\xi}_{0} \times \boldsymbol{B}_{0}\right)=0$, so (22.10) is only a necessary condition for stability, not a sufficient one, and to determine sufficient conditions we must proceed to higher order.

It turns out that $\delta W_{1} \equiv 0$ and

$$
\delta W_{2}=\int_{V} \mathrm{~d} \tau\left\{\left[\boldsymbol{Q}_{\perp 1}\right]^{2}+\left(1+\frac{C}{B_{0}^{2}}\right)\left[Q_{\| 1}\right]^{2}-\sum_{s} m_{s} \iint \frac{B_{0}}{v_{\|}} \frac{\partial f}{\partial \epsilon} \mu^{2}\left\langle Q_{\| 1}\right\rangle^{2} \mathrm{~d} \mu \mathrm{d} \epsilon\right\} .
$$

This is again non-negative, but is zero when

$$
\boldsymbol{Q}_{1}=\nabla \times\left(\xi_{1} \times \boldsymbol{B}_{0}\right)+\nabla \times\left(\xi_{0} \times \boldsymbol{B}_{s}\right)=0
$$

So continuing further, $\delta W_{3} \equiv 0$, and finally

$$
\begin{aligned}
\delta W_{4}= & \int_{V} \mathrm{~d} \tau\left\{\left[Q_{\perp 2}\right]^{2}+\left(1+\frac{C}{B_{0}^{2}}\right)\left[Q_{\| 2}+\frac{C s_{2}-\xi_{0} \cdot \nabla p_{\perp}}{B_{0}\left(1+C / B_{0}^{2}\right)}\right]^{2}\right. \\
& +\frac{\left(C s_{2}-\xi_{0} \nabla p_{\perp}\right)\left[\xi_{0} \cdot \nabla\left(p_{\perp}+B^{2} / 2\right)\right]}{B^{2}+C} \\
& \left.-\sum_{s} m_{s} \iint \frac{B_{0}}{v_{\|}} \frac{\partial f}{\partial \epsilon}\left(\mu B_{0}\right)^{2}\left\langle\frac{Q_{\| 2}}{B}+s_{2}\right\rangle^{2} \mathrm{~d} \mu \mathrm{d} \epsilon\right\} .
\end{aligned}
$$

Several sufficient criteria for stability can be obtained from this last result (Taylor $\&$ Hastie 1965). The most useful and revealing are: $\delta W>0$ if

$$
\left(\nabla_{\|} B\right) \cdot \nabla_{\|}\left(p_{\perp}+B^{2} / 2\right)>0
$$

and

$$
\left(\nabla_{\perp} B\right) \cdot \nabla_{\perp}\left(p_{\perp}+B^{2} / 2\right)>0 .
$$

From (22.15), the maximum plasma pressure $p_{\perp, \max }$ cannot exceed $\left(B_{b}^{2}-B_{c}^{2}\right) / 2$, where $B_{c}^{2} / 2$ is again the magnetic pressure at the minimum of the well but $B_{b}^{2} / 2$ is now the lowest magnetic pressure on the plasma boundary. (In the general equilibria, unlike the special ones, $B$ is not constant along the plasma boundary.)

Note that $\nabla_{\perp}\left(p_{\perp}+B^{2} / 2\right)$ is related to the curvature of lines of force and the condition (22.15) is violated when the diamagnetic plasma currents have so modified the vacuum field that the field curvature is reversed relative to the zero- $\beta$ situation. When $\beta$ is small, the lines of force are convex to the plasma (stable curvature), but, because the plasma is diamagnetic, as the plasma pressure increases, it causes the lines to 'bulge out' and eventually creates unstable curvature. 


\section{Conclusion}

One of the attractions of mirror machines is that one can set up a vacuum mirror field, which is certainly stable, and then introduce plasma gradually. At least at low pressure the plasma should then be well confined - except for the mirror loss cone. (This possibility is not available in toroidal systems such as tokamaks or RFPs, where the plasma itself creates much of the confining field, though it is possible in stellarators.)

Unfortunately, it turns out that plasma in a simple axisymmetric mirror machine is unstable, even at the lowest pressures. This low- $\beta$ instability can be overcome by modifying the field so that there is a non-zero minimum in $B^{2}-$ the 'minimum- $B$ ' fields or magnetic wells. However, to do this one must destroy the symmetry of the field to the extent that it is no longer clear whether the plasma is even confined!

It is important, therefore, that there is a class of equilibria in magnetic wells that can be analysed in considerable detail, using both fluid and particle models, without requiring any knowledge of the actual structure of the magnetic field! This is possible because in minimum- $B$ fields the magnetic isobars (surfaces of constant $B^{2}$ ) form closed nested surfaces that confine plasmas with $p_{\perp}=p_{\perp}(B)$ and $p_{\|}=p_{\|}(B)$.

These special equilibria are unconditionally stable at low $\beta$ in both fluid and particle plasma models. In fact, it appears that the only requirement for low- $\beta$ stability is that the magnetic moment $\mu=v_{\perp}^{2} / 2 B$ of all particles should be invariant. The maximum stable pressure $p_{\perp}$ for the special equilibria is equal to the 'depth' of the magnetic well - the difference in $B^{2}$ between its minimum and its value on the last closed magnetic isobar.

To analyse general equilibria in mirrors, we introduced a Hamiltonian description of the average (over the bounce motion between mirrors) guiding centre drift. This showed, as one would expect, that an equilibrium distribution must be a function of the constants of motion on each field line $f=f(\mu, \epsilon, \alpha, \beta)$. It also allowed us to calculate the necessary and sufficient conditions for stability of general low- $\beta$ equilibria in mirror machines.

We also introduced an important approximate method for calculating the maximum stable $\beta$ for general equilibria. This so-called 'maximal ordering' is important in many areas of plasma confinement, in both mirror and toroidal systems. When applied to mirrors, it showed that for general equilibria the maximum stable $\beta$ is again related to the 'depth' of the magnetic well - in this case the difference in $B^{2}$ between its minimum and its lowest value on the plasma boundary (which in general is not a magnetic isobar).

However, despite their attraction in terms of control, stability and susceptibility to theoretical analysis, mirror machines suffer from an inevitable loss of plasma due to collisional scattering into the mirror loss cones. Despite heroic attempts to plug these losses by complex multiple mirrors, electrostatic barriers, thermal barriers, radiation barriers, etc., it seems that the loss cannot be reduced to a level acceptable for fusion reactors - the original motivation for studying mirror machines. However, they remain a valuable research tool and are used (in an independent development) as 'magnetic traps' for studies of single atoms and Bose-Einstein condensates, etc.

\section{Part IV}

\section{Ballooning modes}

The theory of ballooning modes is one of the most intriguing topics in plasma stability theory. It represents a significant step forwards from the study of idealised 
configurations such as the plane slab and infinite circular cylinder - in which ballooning modes do not exist - to more realistic toroidal systems with arbitrary cross-section and arbitrary aspect ratio - in which ballooning modes are important. They set a maximum $\beta$ for plasma stability in a tokamak and fill a gap that previously existed in the theory of toroidal plasmas.

But before we embark on the investigation of ballooning modes, we should look at the theory of instabilities in an infinite circular cylinder, even though that investigation cannot be extended to toroidal systems.

\subsection{Stability of plasma in circular cylinders}

A full linear stability analysis can be carried out for plasma in an infinite circular cylinder. This is possible because any linear perturbation can be reduced to a sum over independent one-dimensional Fourier modes $\boldsymbol{\xi}(r) \exp [\mathrm{i}(m \theta+k z)]$, as in the classic analysis of Newcomb (1960).

If, following Newcomb, we consider a single Fourier mode $(m, k)$ and drop the exponential factor, the displacement can be expressed in terms of the real variables

$$
\begin{gathered}
\xi=\xi_{r}, \\
\eta=\nabla \cdot \xi-\frac{1}{r} \frac{\mathrm{d}}{\mathrm{d} r}\left(r \xi_{r}\right)=\frac{\mathrm{i} m}{r} \xi_{\theta}+\mathrm{i} k \xi_{z}, \\
\zeta=\mathrm{i}(\boldsymbol{\xi} \times \boldsymbol{B})_{r}=\mathrm{i} \xi_{\theta} B_{z}-\mathrm{i} \xi_{z} B_{\theta} .
\end{gathered}
$$

The usual cylindrical components $\xi_{\theta}$ and $\xi_{z}$ are then given by

$$
\xi_{\theta}=-\mathrm{i} \frac{k r \zeta+r B_{\theta} \eta}{k r B_{z}+m B_{\theta}}, \quad \xi_{z}=\mathrm{i} \frac{m \zeta-r B_{z} \eta}{k r B_{z}+m B_{\theta}} .
$$

After some algebra, the energy functional for isotropic pressure $\delta W_{1}$ (see (19.7)),

$$
\delta W_{1}\left(\boldsymbol{\xi}^{*}, \boldsymbol{\xi}\right)=\frac{1}{2} \int_{V} \mathrm{~d} \tau\left\{|\boldsymbol{Q}|^{2}-\boldsymbol{j} \cdot \boldsymbol{\xi}^{*} \times \boldsymbol{Q}+\left(\nabla \cdot \boldsymbol{\xi}^{*}\right) \boldsymbol{\xi} \cdot \nabla p+\gamma p(\nabla \cdot \boldsymbol{\xi})^{2}\right\}
$$

can be reduced to a form that can easily be minimised over $\eta$ and $\zeta$. After this, $\delta W_{1}$ depends only on the radial displacement $\xi$ and reduces to

$$
\delta W_{1}(\xi)=\frac{\pi}{2} \int_{a}^{b} \mathrm{~d} r\left[f\left(\frac{\mathrm{d} \xi}{\mathrm{d} r}\right)^{2}+g \xi^{2}\right]
$$

where

$$
\begin{gathered}
f=\frac{r\left(k r B_{z}+m B_{\theta}\right)^{2}}{k^{2} r^{2}+m^{2}}, \\
g=\frac{1}{r} \frac{\left(k r B_{z}-m B_{\theta}\right)^{2}}{k^{2} r^{2}+m^{2}}+\frac{1}{r}\left(k r B_{z}+m B_{\theta}\right)^{2}-\frac{2 B_{\theta}}{r} \frac{\mathrm{d}}{\mathrm{d} r}\left(r B_{\theta}\right)-\frac{\mathrm{d}}{\mathrm{d} r}\left(\frac{k^{2} r^{2} B_{z}^{2}-m^{2} B_{\theta}^{2}}{k^{2} r^{2}+m^{2}}\right) .
\end{gathered}
$$

As explained in Newcomb (1960), examination of (24.6)-(24.8) for different $(m, k)$ shows that a cylindrical plasma will be stable for all $(m, k)$ if it is stable for $m=1$, $-\infty<k<\infty$ and for $m=0, k \rightarrow 0$. 


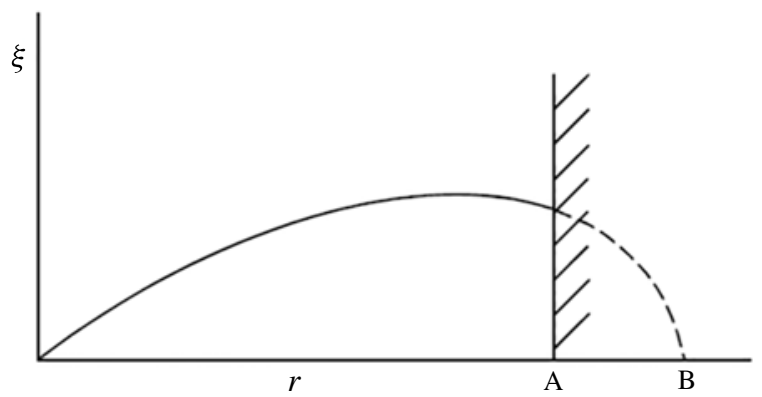

FIGURE 55. Projected displacement. $\mathrm{A}$ is the actual wall position and $\mathrm{B}$ is the hypothetical position needed for marginal stability.

Equation (24.6) is a standard form, and minimisation over $\xi$ leads to the EulerLagrange equation

$$
\frac{\mathrm{d}}{\mathrm{d} r}\left(f \frac{\mathrm{d} \xi}{\mathrm{d} r}\right)-g \xi=0 .
$$

An important feature of (24.9) is that there is a singular point at the 'resonant surface' where $k r B_{z}+m B_{\theta}=0$ and $f$ vanishes.

Suppose, first, that there is no resonant surface within the boundary at $r=b$ (which we take to be perfectly conducting), and that we integrate (i.e. 'project') a solution of (24.9) from $r=0, \xi=0$ towards the boundary at $r=b$. (Of course, equation (24.9) has two independent solutions, but only one vanishes at the origin.) Now, if this projected solution does not vanish anywhere before reaching the boundary (see figure 55), then the plasma is stable for that $m$ and $k$. Newcomb proves this mathematically, but we can see that it must be true by the following simple argument.

The Euler-Lagrange equation (24.9) is also the equation for marginal stability $\left(\omega^{2}=0\right)$ and it is clear that, if our projected solution does not vanish before reaching the wall, then for the plasma to be marginally stable we would have to move the wall (which has a stabilising effect) to a larger radius. Hence, with the wall at its actual radius, the plasma must be stable.

If there is a single resonant surface in the plasma, the argument is only a little more complicated. In this case we 'project' two solutions of the marginal equation, one outwards from $r=0, \xi=0$ as before and the other inwards from the wall $r=b$, $\xi=0$. Then if neither of them vanishes before they reach the singular surface, we have exactly the situation (see figure 15) that we discussed in $\$ 5.1$. From that analysis we know that an ideal plasma is stable. Furthermore, we now know that a resistive plasma is also stable if $\Delta^{\prime}<0$, where $\Delta^{\prime}$ is the change in logarithmic derivative of $\xi$, or the change in the small solution of (24.9) across the resonant surface (see (5.6)).

Before we turn to toroidal plasmas, note that the analysis of circular plasmas requires only the solution of the single differential equation (24.9).

\section{Modes in toroidal systems}

In a toroidal plasma, perturbations cannot be broken down into independent onedimensional Fourier modes, though in an axisymmetric torus they can be reduced to a sum over independent two-dimensional modes $\xi(r, \theta) \exp (i n \zeta)$, where $\zeta$ is the toroidal angle and $\theta$ is the poloidal angle. But, in fact, ballooning modes have a completely different structure from the modes in a cylinder. They involve many, strongly coupled, 


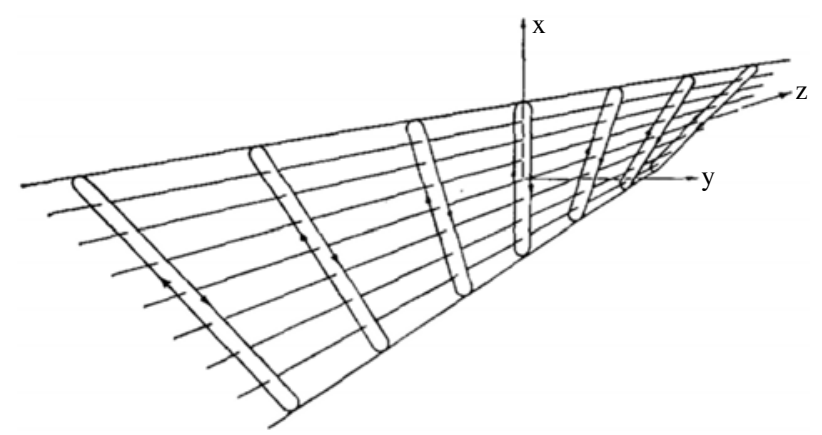

FIGURE 56. Twisted slicing mode (from Roberts \& Taylor 1965).

Fourier modes and therefore cannot be dealt with by retaining a limited number of such modes.

The principal characteristic of ballooning modes is that they have short wavelength perpendicular to the magnetic field but long wavelength parallel to it. Specifically, $k_{\perp} a \ll 1$ and $k_{\|} R \sim 1$, where $a$ and $R$ are the minor and major radii of the torus. (Note that, although $k_{\|}$is small, it is not zero, as in a flute mode; see $\S 17$.) Because of the long wavelength parallel to $\boldsymbol{B}$, the stabilising effect of the magnetic field on ballooning modes is weak and they are therefore among the most pervasive instabilities in a toroidal plasma. Also, because of the short transverse wavelength, they are difficult to compute directly.

Ballooning modes occur in most plasma models - ideal MHD, resistive MHD, gyrofluid, gyrokinetic, etc. However, the theory is most easily explained in MHD, and we shall concentrate on this application.

In a torus, as we move along a field line, it passes through regions of stable curvature $(\kappa \cdot \nabla p<0$, on the inner equator) and regions of unstable curvature $(\kappa \cdot \nabla p>0$, on the outer equator). A perturbation aligned with the field therefore tends to be largest on the outer equator, where it bulges out (as does a weak spot in a balloon!). However, this ballooning is opposed by the field line bending that accompanies it, and the mode is a balance between these two effects. The amount of field line bending associated with a given perturbation depends on the magnetic shear - which therefore plays an important role in ballooning modes.

Historically, the significance of the ballooning effect was first noted in connection with drift waves by Taylor (1977), though in a form that bears little resemblance to the later theory. The material in the following sections is based upon various original sources. The idea that the coupling of almost degenerate Fourier modes on adjacent surfaces could produce a radially extended mode was introduced by Roberts \& Taylor (1965), who referred to the extended modes as 'twisted slices' (see figure 56), but they envisaged a nonlinear coupling rather than toroidal coupling. (This oversight probably delayed the theory of ballooning modes by 20 years!) The full theory for high- $n$ ballooning modes was given by Connor et al. (1978, 1979a,b). Independent approaches were developed by Glasser (1979) and Lee \& Dam (1979).

\section{High mode number perturbations in an axisymmetric torus}

As we mentioned above, in an axisymmetric torus perturbations can be reduced to independent modes $\sim \boldsymbol{\xi}(r, \theta) \exp (\mathrm{i} n \zeta)$ with toroidal mode number $n$. We will consider first the limit of large toroidal mode number $n$ and show that in this limit the oscillations on each magnetic surface are decoupled and independent. 
The lowest order in an expansion in $1 / \sqrt{n}$ of the scalar pressure energy functional $\delta W$ then defines a local frequency $\omega^{2}(\Psi)$ on each surface, and determines the structure of the mode along the magnetic field $\boldsymbol{B}$. Higher orders in the expansion determine the cross-field structure and relate the local frequency $\omega^{2}(\Psi)$ to the global frequency $\Omega^{2}$ - which finally determines stability of the system.

The important discovery is that the local frequency $\omega^{2}(\Psi)$, which requires only the solution of a one-dimensional ordinary differential equation, nevertheless contains all the information necessary for the complete solution of the global problem. The difficulty is that, before we can calculate anything, we must find a representation for the perturbation that reconciles the short perpendicular and long parallel wavelength with toroidal periodicity in a sheared magnetic field.

To represent short-wavelength disturbances, $\phi$, in a medium that changes on a much longer scale, one normally uses an 'eikonal' or Wentzel-Kramers-Brillouin-Jeffreys (WKBJ) form,

$$
\phi \sim F \exp (\mathrm{i} n S),
$$

where $F$ and $S$ vary slowly, but the exponential varies rapidly when $n$ is large. In the present case, the requirement for long parallel wavelength means that we must have

$$
\boldsymbol{B} \cdot \nabla S=0,
$$

but this is incompatible with periodicity around the torus in a sheared field. For, if $S$ is constant along a line of force, then, following a line once around the torus, one must have

$$
S(\Psi, \theta)-S\left(\Psi, \theta+\frac{2 \pi}{q(\Psi)}\right)=\text { integer } \times \frac{2 \pi}{n} .
$$

Owing to the shear, $q(\Psi)$ varies from one magnetic surface to another, so this condition cannot be satisfied everywhere without $S$ being discontinuous.

In the next section we show that a new representation allows us to combine the advantages of the eikonal with long parallel wavelength and toroidal periodicity.

\subsection{Ballooning representation}

After we have selected a single toroidal mode $\propto \exp (\mathrm{in} \zeta)$, the calculation of linear perturbations in an axisymmetric plasma reduces to an eigenvalue equation of the form

$$
\mathcal{L}(\theta, x) \phi(\theta, x)=\lambda \phi(\theta, x),
$$

where $x$ is a radial flux coordinate. The operator $\mathcal{L}$ and the function $\phi$ are both periodic in poloidal angle $\theta$.

Now write

$$
\phi(\theta, x)=\sum_{m} \mathrm{e}^{-\mathrm{i} m \theta} \int_{-\infty}^{\infty} \mathrm{e}^{\mathrm{i} m \eta} \hat{\phi}(\eta, x) \mathrm{d} \eta .
$$

This ensures that $\phi$ is periodic in $\theta: \hat{\phi}$ does not need to be periodic. This 'ballooning transform' really combines three steps:

(i) $\phi \rightarrow$ a Fourier sum $\sum_{m} a_{m}(x) \exp (-\mathrm{i} m \theta)$;

(ii) $a_{m}(x)$ is extended to a function $a(s, x)$ that coincides with $a_{m}(x)$ when $s=m$;

(iii) $a(s, x)$ is represented by a Fourier integral in the coordinate $\eta$.

By direct substitution, it can be shown that $\hat{\phi}$ satisfies the same equation as $\phi$,

$$
\mathcal{L}(\eta, x) \hat{\phi}(\eta, x)=\lambda \hat{\phi}(\eta, x),
$$

and has the same eigenvalues. 
We have effectively taken a Fourier transform in step (i) and then reversed it in step (ii). This is precisely why (26.6) is formally identified with (26.4). However the first transform was a Fourier sum and the second was a Fourier integral; consequently $\hat{\phi}$ does not have to be periodic.

The extent to which (26.5) is mathematically a true reversible transform, in the spirit of Fourier, Abel, etc., is discussed at length in Connor et al. (1979b), Hazeltine et al. (1981), Dewar \& Glasser (1983) and Hazeltine \& Newcomb (1990). This highly mathematical discussion is not appropriate here; it is sufficient to note that, as we will see below, when we introduce a WKBJ eikonal approximation for $\hat{\phi}$ in (26.5), we obtain a stability criterion, and an estimate of the growth rates and the profiles of unstable modes.

Another interpretation of (26.6) is that we have replaced the original stability problem, with its incompatible periodicity and wavelength requirements, by a fictitious problem in an infinite domain with no periodicity requirements, but which has the same eigenvalues. The operator is unchanged, so the short perpendicular and long parallel wavelength properties of the solution are unchanged.

The point of all of this is that, unlike $\phi, \hat{\phi}(\eta, x)$ can be represented in eikonal form,

$$
\hat{\phi}(\eta, x)=F(\eta, x) \exp (\mathrm{i} n S),
$$

with $\boldsymbol{B} \cdot \nabla S=0$ and both $F$ and $S$ slowly varying.

This perturbation has essentially the same form as the Roberts \& Taylor (1965) 'twisted slice' quasi-mode, but this is no longer the actual perturbation. We will see later that the actual perturbation is a superposition of such 'slices'.

Of course, as we no longer have the periodic boundary condition, we need a replacement boundary condition for $\hat{\phi}(\eta, x)$. This follows from the requirement that the transformation back to $\phi(\eta, x)$ must lead to a physically acceptable function. Consequently, $\phi$ must tend to zero sufficiently fast as $|\eta| \rightarrow 0$ for the integrals in (26.5) to converge.

\section{Magnetohydrodynamic stability in a torus}

In an axisymmetric torus the equilibrium field can be written as

$$
\boldsymbol{B}=\nabla \Psi \times \nabla \zeta+I(\Psi) \nabla \zeta,
$$

where $\Psi$ defines the magnetic surface and $\zeta$ is the toroidal angle around the torus. The first term gives the poloidal field and the second the toroidal component (compare with the Grad-Shafranov equilibrium in (2.19)).

In this section we denote the poloidal angle by $\chi$ to conform with Connor et al. $(1979 b)$. Then, in the coordinates $(\Psi, \chi, \zeta)$, we have the line element

$$
\mathrm{d} s^{2}=\frac{1}{\left(R B_{\chi}\right)^{2}}(\mathrm{~d} \Psi)^{2}+\left(J B_{\chi}\right)^{2}(\mathrm{~d} \chi)^{2}+R^{2}(\mathrm{~d} \zeta)^{2},
$$

and the volume element $\mathrm{d} \tau=J \mathrm{~d} \Psi \mathrm{d} \chi \mathrm{d} \zeta$, with the Jacobian $J=|\nabla \Psi \cdot \nabla \chi \times \nabla \zeta|^{-1}$. A field line is defined by $\Psi=$ constant, $\chi=\chi(\zeta)$, where

$$
\frac{\mathrm{d} \zeta}{\mathrm{d} \chi}=\frac{I J}{R^{2}} \equiv v(\Psi)
$$


and the safety factor is

$$
q(\Psi)=\frac{1}{2 \pi} \oint v \mathrm{~d} \chi
$$

Turning now to the question of stability, we again take the scalar pressure energy principle,

$$
\delta W_{1}=\frac{1}{2} \int Q^{2}-\boldsymbol{j} \cdot \boldsymbol{Q} \times \boldsymbol{\xi}+(\boldsymbol{\xi} \cdot \nabla p)(\nabla \cdot \boldsymbol{\xi})+\gamma p(\nabla \cdot \boldsymbol{\xi})^{2} \mathrm{~d} \tau,
$$

and describe a perturbation $\xi$ by the three components:

$$
\begin{aligned}
X & =R B_{\chi} \xi_{\Psi}, \\
U & =\left(\frac{\xi_{\zeta}}{R}-\frac{I}{R^{2} B_{\chi}} \xi_{\chi}\right), \\
Z & =\frac{\xi_{\chi}}{B_{\chi}} .
\end{aligned}
$$

Here $X$ is the displacement normal to flux surfaces, $U$ is the displacement tangential to a surface and normal to the field, and $Z$ is the displacement in the poloidal direction. In terms of these components, the perturbed energy becomes

$$
\begin{aligned}
\delta W= & \frac{1}{2} \int J \mathrm{~d} \Psi \mathrm{d} \zeta \mathrm{d} \chi\left\{\frac{B^{2}}{R^{2} B_{\chi}^{2}}\left|k_{\|} X\right|^{2}+\frac{R^{2}}{J^{2}}\left|\frac{\partial U}{\partial \chi}-I \frac{\partial}{\partial \Psi}\left(\frac{J X}{R^{2}}\right)\right|^{2}\right. \\
& +B_{\chi}^{2}\left|\mathrm{i} n U+\frac{\partial X}{\partial \Psi}+\frac{j_{\zeta}}{R B_{\chi}^{2}} X\right|^{2}-2 K|X|^{2} \\
& \left.+\gamma p\left|\frac{1}{J} \frac{\partial}{\partial \Psi}(J X)+\mathrm{i} n U+\mathrm{i} B k_{\|} Z\right|^{2}\right\}
\end{aligned}
$$

where

$$
\mathrm{i} k_{\|}=\frac{1}{J B}\left(\frac{\partial}{\partial \chi}+\mathrm{i} n v\right), \quad K=\frac{I I^{\prime}}{R^{2}} \frac{\partial}{\partial \Psi} \ln R-\frac{j_{\zeta}}{R} \frac{\partial}{\partial \Psi} \ln \left(J B_{\chi}\right),
$$

and a prime denotes the derivative with respect to $\Psi$. Minimisation of $\delta W$ over $Z$ makes the last term of (27.9) zero, corresponding to $\nabla \cdot \xi=0$. Then minimisation over $U$ can be carried out as an expansion in $1 / n$. Correct to order $1 / n$ this requires

$$
\mathrm{i} n U+\frac{\partial X}{\partial \Psi}+X\left(\frac{p^{\prime}}{B^{2}}+\frac{v^{\prime}}{v} \frac{I^{2}}{R^{2} B^{2}}\right)+\frac{I^{2}}{\nu R^{2} B^{2}} J B k_{\|}\left(\frac{1}{n} \frac{\partial X}{\partial \Psi}\right)=0,
$$

which reduces $\delta W$ to a form involving only the displacement $X$,

$$
\begin{aligned}
\delta W= & \pi \int \mathrm{d} \Psi \mathrm{d} \chi\left\{\frac{J B^{2}}{R^{2} B_{\chi}^{2}}\left|k_{\|} X\right|^{2}+\frac{R^{2} B_{\chi}^{2}}{J B^{2}}\left|\frac{1}{n} \frac{\partial}{\partial \Psi}\left(J B k_{\|} X\right)^{2}\right|^{2}\right. \\
& -\frac{2 J}{B^{2}} p^{\prime}\left[|X|^{2} \frac{\partial}{\partial \Psi}\left(p+\frac{B^{2}}{2}\right)-\frac{\mathrm{i} I}{J B^{2}} \frac{\partial}{\partial \chi}\left(\frac{B^{2}}{2}\right) \frac{X^{*}}{n} \frac{\partial X}{\partial \Psi}\right] \\
& \left.+\frac{X^{*}}{n} J B k_{\|}\left(X \sigma^{\prime}\right)-\frac{1}{n}\left[P^{*} J B k_{\|} Q+P J B k_{\|}^{*} Q^{*}\right]\right\},
\end{aligned}
$$


where

$$
\begin{aligned}
& P=X \sigma-\frac{B_{\chi}^{2}}{v B^{2}} \frac{I}{n} \frac{\partial}{\partial \Psi}\left(J B k_{\|} X\right), \\
& Q=\frac{X p^{\prime}}{B^{2}}+\frac{I^{2}}{v R^{2} B^{2}} \frac{1}{n} \frac{\partial}{\partial \Psi}\left(J B k_{\|} X\right), \\
& \sigma=\frac{I p^{\prime}}{B^{2}}+I^{\prime} .
\end{aligned}
$$

To test for stability, we should now minimise (27.12) with respect to all functions $X(\Psi, \chi)$ periodic in $\chi$. However, in order to also derive a measure of the growth rate, or frequency, of the ballooning modes, we need to introduce a suitable normalisation for $X$. We take

$$
\pi \int J \mathrm{~d} \Psi \mathrm{d} \chi\left\{\frac{|X|^{2}}{R^{2} B_{\chi}^{2}}+\left(\frac{R B_{\chi}}{B}\right)^{2}\left|\frac{1}{n} \frac{\partial X}{\partial \Psi}\right|^{2}\right\}=1,
$$

which is the kinetic energy in the motion transverse to $\boldsymbol{B}$. (This is more convenient than the total kinetic energy because it does not involve $U$ or $Z$.)

Then, minimising (27.12) subject to (27.16) gives the Euler-Lagrange equation

$$
\begin{aligned}
J B k_{\|} & \left\{\frac{1}{J R^{2} B_{\chi}^{2}}\left[1-\left(\frac{R^{2} B_{\chi}^{2}}{B}\right)^{2} \frac{1}{n^{2}} \frac{\partial^{2}}{\partial \Psi^{2}}\right] J B k_{\|} X\right\}-\frac{2 J}{B^{2}} X p^{\prime} \frac{\partial}{\partial \Psi}\left(p+\frac{B^{2}}{2}\right) \\
& +\frac{1}{n} \frac{\partial X}{\partial \Psi} \frac{p^{\prime} I}{B^{4}} \frac{\partial B^{2}}{\partial \chi}-\frac{1}{n} J B k_{\|}\left[\frac{\partial}{\partial \Psi}\left(\frac{R^{2} B_{\chi}^{2}}{J B^{2}}\right) \frac{1}{n} \frac{\partial}{\partial \Psi}\left(J B k_{\|} X\right)\right] \\
& +\frac{1}{n} J B k_{\|}\left(\sigma^{\prime} X\right)-\frac{\sigma}{n} J B k_{\|} Q-\frac{p^{\prime}}{n B^{2}} J B k_{\|} P \\
& -\frac{1}{n} J B k_{\|}\left\{\frac{1}{n} \frac{\partial}{\partial \Psi}\left[\frac{I B_{\chi}^{2}}{v B^{2}} J B k_{\|} Q\right]\right\}+\frac{1}{n} J B k_{\|}\left\{\frac{1}{n} \frac{\partial}{\partial \Psi}\left[\frac{I^{2}}{v R^{2} B^{2}} J B k_{\|} P\right]\right\} \\
= & \Omega^{2}\left[\frac{J}{R^{2} B_{\chi}^{2}} X-\frac{J R^{2} B_{\chi}^{2}}{B^{2}} \frac{1}{n^{2}} \frac{\partial^{2} X}{\partial \Psi^{2}}-\frac{1}{n} \frac{\partial}{\partial \Psi}\left(\frac{J R^{2} B_{\chi}^{2}}{B^{2}}\right) \frac{1}{n} \frac{\partial X}{\partial \Psi}\right] .
\end{aligned}
$$

Recalling that

$$
\mathrm{i} k_{\|}=\frac{1}{J B}\left(\frac{\partial}{\partial \chi}+\mathrm{i} n v\right),
$$

we see that (27.17) is a second-order partial differential equation in $\partial / \partial \Psi$ and $\partial / \partial \chi$ for the periodic function $X(\Psi, \chi)$, where $X\left(\Psi, \chi+\chi_{0}\right)=X(\Psi, \chi)$ and $\chi_{0}=\oint \mathrm{d} \chi$.

We now introduce the ballooning transformation,

$$
X(\Psi, \chi)=\sum_{m} \exp \left(-\frac{2 \pi \mathrm{i} m \chi}{\chi_{0}}\right) \int_{-\infty}^{\infty} \mathrm{d} y \exp \left(\frac{2 \pi \mathrm{i} m y}{\chi_{0}}\right) \hat{X}(\Psi, y) .
$$

This converts (27.17) into an identical equation for $\hat{X}$, with $\chi \rightarrow y$, but now in the infinite domain $-\infty<y<\infty$ and free of periodicity constraints. So we can write $\hat{X}$ in eikonal form,

$$
\hat{X}(\Psi, y)=F(\Psi, y) \exp \left(-\mathrm{i} n \int_{y_{0}}^{y} v \mathrm{~d} y\right) .
$$

Note the appearance here of the arbitrary phase $y_{0}$, which will be discussed below. 
If we restore the toroidal mode number $\exp (\mathrm{i} n \zeta)$, equation (27.20) is equivalent to the eikonal (26.1), Fe ${ }^{\mathrm{i} n S}$, with $S=\zeta-\int \nu \mathrm{d} \chi$ and $\boldsymbol{B} \cdot \nabla S=0$. We have therefore reached the point where $\hat{X}$ is in eikonal form, with $F$ and $S$ varying slowly compared to the rapid variation across the field of the phase $\exp (\mathrm{i} n S)$.

Now we invoke large $n$, and introduce two length scales transverse to the magnetic surfaces: (i) the equilibrium scale, which we continue to denote by $\Psi$, and (ii) a more rapid scale $x=\sqrt{n}\left(\Psi-\Psi_{0}\right)$, where $\Psi_{0}$ is to be identified later. This scale is related to the distance between rational surfaces on which $q(\Psi)=m / n$. The separation between these surfaces tends to zero as $n \rightarrow \infty$.

Then, writing the transformed equation (27.17) as

$$
\left(L+\Omega^{2} M\right) F=0,
$$

we expand in powers of $1 / \sqrt{n}$,

$$
\begin{aligned}
L & =L_{0}+\frac{1}{\sqrt{n}} L_{1}+\frac{1}{n} L_{2}+\cdots, \\
M & =M_{0}+\frac{1}{\sqrt{n}} M_{1}+\frac{1}{n} M_{2}+\cdots .
\end{aligned}
$$

The lowest order approximation is

$$
\left[L_{0}+\omega^{2}\left(\Psi, y_{0}\right) M_{0}\right] F_{0}=0,
$$

where

$$
\begin{aligned}
L_{0} F= & \frac{\partial}{\partial y}\left\{\frac{1}{J R^{2} B_{\chi}^{2}}\left[1+\left(\frac{R^{2} B_{\chi}^{2}}{B} \int_{y_{0}}^{y} v^{\prime} \mathrm{d} y\right)^{2}\right] \frac{\partial F}{\partial y}\right\} \\
& +F\left\{\frac{2 J p^{\prime}}{B^{2}} \frac{\partial}{\partial \Psi}\left(p+\frac{B^{2}}{2}\right)-\frac{I p^{\prime}}{B^{4}}\left(\int_{y_{0}}^{y} v^{\prime} \mathrm{d} y\right) \frac{\partial B^{2}}{\partial y}\right\}, \\
M_{0} F= & \frac{J}{R^{2} B_{\chi}^{2}}\left[1+\left(\frac{R^{2} B_{\chi}^{2}}{B} \int_{y_{0}}^{y} v^{\prime} \mathrm{d} y\right)^{2}\right] F .
\end{aligned}
$$

(Higher-order operators $L_{1}, M_{1}, L_{2}$ and $M_{2}$ will be introduced later.) Note that $L_{0}$ is a one-dimensional differential operator in the extended coordinate $y$-it depends only parametrically on $\Psi$ and $y_{0}$.

The zeroth order equation (27.24) has the structure

$$
\frac{\mathrm{d}}{\mathrm{d} y}\left(a+b \int_{y_{0}}^{y} v^{\prime} \mathrm{d} y^{\prime}\right)^{2} \frac{\mathrm{d} F_{0}}{\mathrm{~d} y}+p^{\prime}\left(c+d \int_{y_{0}}^{y} v^{\prime} \mathrm{d} y^{\prime}\right) F_{0}=e \omega^{2}\left[a+b\left(\int_{y_{0}}^{y} v^{\prime} \mathrm{d} y^{\prime}\right)^{2}\right] F_{0},
$$

on each magnetic surface $\Psi$. The coefficients $a, b, c, d$ and $e$ are periodic in $y$ and defined on each surface by the equilibrium. The terms $\int v^{\prime} \mathrm{d} y$ increase indefinitely with $y$ and show the strong effect of magnetic shear. The term $c$ represents the destabilising normal curvature, and $d$ is related to the geodesic curvature. 
Equation (27.27) is a second-order differential equation for $\omega^{2}\left(\Psi, y_{0}\right)$ and can be readily solved numerically. To lowest order, therefore, the oscillations on each surface are decoupled and each has a frequency $\omega\left(\Psi, y_{0}\right)$ that depends on the properties (curvature, shear, etc.) of that surface and the, as yet unknown, $y_{0}$.

To calculate $\omega^{2}\left(\Psi, y_{0}\right)$, we need to apply the boundary conditions $F_{0} \rightarrow 0$ as $|y| \rightarrow \infty$. Equation (27.24) has two independent solutions at large $|y|$, but if $\omega^{2}<0$ one is exponentially growing and can be rejected. In this case, one finds a discrete unstable eigenvalue spectrum. If $\omega^{2}>0$ both solutions behave like $(1 / y) \exp (\mathrm{i} \omega y)$ at large $|y|$ and both must be retained. Consequently, any positive $\omega^{2}$ is acceptable and in this case there is a continuous spectrum of stable modes - as there is in a cylindrical plasma (Grad 1973).

As (27.24) is a differential equation in $y$ alone, any solution can be multiplied by an arbitrary function of $x$, so the full solution in lowest order should be

$$
\mathcal{F}_{0}=A(x) F_{0}\left(y ; \Psi, y_{0}\right)
$$

It must be emphasised that, at this point, although the 'local' eigenvalue $\omega^{2}\left(\Psi, y_{0}\right)$ and the eigenfunction $F\left(y ; \Psi, y_{0}\right)$ (which gives the perturbation along the field) can readily be computed, we so far have no information on the profile $A(x)$ (perpendicular to the magnetic surfaces) or on the correct value for $y_{0}$, or on the relation of $\omega^{2}\left(\Psi, y_{0}\right)$ to the global eigenvalue $\Omega^{2}$ - which ultimately determines global stability. We will show later that, when the theory is developed to higher order in $1 / \sqrt{n}$, these unknown factors are determined uniquely, and that all of the information necessary to do so is already implicit in $\omega^{2}\left(\Psi, y_{0}\right)$.

\subsection{Higher-order theory}

As emphasised at the end of the previous section, to complete the theory of MHD stability in a torus, we still have to

(i) find the 'envelope' $A(x)$, which defines the structure of a ballooning mode across the magnetic surfaces,

(ii) find $\Psi_{0}$, which locates the surface on which the perturbation is maximum,

(iii) find $y_{0}$, which locates the poloidal angle at which the perturbation is maximum,

(iv) relate the local $\omega^{2}\left(\Psi, y_{0}\right)$ to the global eigenvalue $\Omega^{2}$, which determines overall stability.

To find these quantities, we must proceed to higher order in the $1 / \sqrt{n}$ expansion of (27.21). This involves the operators $L_{1}, L_{2}, M_{1}$ and $M_{2}$. The operators $L_{1}$ and $M_{1}$ are

$$
L_{1}=-\mathrm{i} \frac{1}{v^{\prime}\left(y_{0}\right)} \frac{\partial L_{0}}{\partial y_{0}} \frac{\partial}{\partial x}, \quad M_{1}=-\mathrm{i} \frac{1}{v^{\prime}\left(y_{0}\right)} \frac{\partial M_{0}}{\partial y_{0}} \frac{\partial}{\partial x},
$$

and the next order equation is

$$
\left(L_{0}+\omega^{2} M_{0}\right) F_{1}+\left(L_{1}+\omega^{2} M_{1}\right) F_{0}=0 .
$$

This can be solved for $F_{1}$ only if the integrability condition

$$
\int_{-\infty}^{\infty} F_{0}\left(L_{1}+\omega^{2} M_{1}\right) F_{0} \mathrm{~d} y=0
$$


is satisfied. From the form of $L_{1}$ and $M_{1}$, it can be shown that this is equivalent to the constraint

$$
\frac{\partial}{\partial y_{0}} \omega^{2}\left(\Psi, y_{0}\right)=0
$$

This fixes the undetermined parameter $y_{0}$ : on each magnetic surface it is at a minimum of $\omega^{2}\left(\Psi, y_{0}\right)$. Usually this is on the outer equator of the torus (Cooper 1988; Taylor 2012).

Proceeding further in the $1 / \sqrt{n}$ expansion, in second order an integrability condition is obtained involving the operators $M_{2}$ and $L_{2}$ :

$$
\int\left[F_{0}\left(L_{1}+\omega^{2} M_{1}\right) F_{1}+F_{0}\left(L_{2}+\omega^{2} M_{2}\right) F_{0}+n\left(\Omega^{2}-\omega^{2}\right) F_{0} M_{0} F_{0}\right] \mathrm{d} y=0 .
$$

If we choose $\Psi_{0}$ to be at a minimum, with respect to $\Psi$, of $\omega^{2}\left(\Psi, y_{0}\right)$ (with $y_{0}$ fixed as above), then expanding $\omega^{2}(\Psi)$ about this minimum, and using the properties of the operators $L_{1}, M_{1}, L_{2}$ and $M_{2}$, one eventually obtains from (27.33) an equation for $A(x)$ :

$$
\frac{\partial^{2} \omega_{0}^{2}}{\partial y_{0}^{2}} \frac{\mathrm{d}^{2} A}{\mathrm{~d} x^{2}}+\left(v^{\prime}\left(y_{0}\right)\right)^{2}\left[2 n\left(\Omega^{2}-\omega_{0}^{2}\right)-\frac{\partial^{2} \omega^{2}}{\partial \Psi^{2}} x^{2}\right] A(x)=0 .
$$

Therefore

$$
A(x)=\exp \left[-\frac{1}{2}\left|v^{\prime}\left(y_{0}\right)\right|\left(\frac{\partial^{2} \omega^{2} / \partial \Psi^{2}}{\partial^{2} \omega^{2} / \partial y_{0}^{2}}\right)^{1 / 2} x^{2}\right]
$$

and

$$
\Omega^{2}=\omega_{0}^{2}+\frac{1}{2 n\left|\nu^{\prime}\left(y_{0}\right)\right|}\left(\frac{\partial^{2} \omega^{2}}{\partial \Psi^{2}} \frac{\partial^{2} \omega^{2}}{\partial y_{0}^{2}}\right)^{1 / 2}
$$

This completes the theory, and shows that all the information needed to do so was already contained in the lowest-order quantity $\omega^{2}\left(\Psi, y_{0}\right)$. In practice, therefore, we have only to compute solutions of the differential equation (27.24). Note also from (27.36) that ballooning modes become more unstable as $n \rightarrow \infty$. This is precisely the region that is most difficult to calculate by direct simulation and explains why ballooning theory filled an important gap in the MHD theory of toroidal plasmas. (Of course, in reality MHD theory breaks down at very high $n$ due to Larmor radius and other non-ideal effects.)

\section{Applications}

The first application of the theory was to a large-aspect-ratio torus, by Connor et al. (1978). In this so-called ' $s-\alpha$ ' model, the coefficients of (27.27) can be expressed analytically and it becomes

$$
\frac{\mathrm{d}}{\mathrm{d} y}\left[1+\left(s\left(y-y_{0}\right)-\alpha \sin y\right)^{2}\right] \frac{\mathrm{d} F}{\mathrm{~d} y}+\alpha\left[\cos y+\sin y\left(s\left(y-y_{0}\right)-\alpha \sin y\right)\right] F=0 .
$$

Here $y_{0}$ is the parameter that locates the poloidal angle at which the mode amplitude is maximum. It must be chosen to give the largest region of instability. The parameter $s$ is a measure of the mean shear,

$$
s=\frac{\mathrm{d}(\log q)}{\mathrm{d}(\log r)}
$$




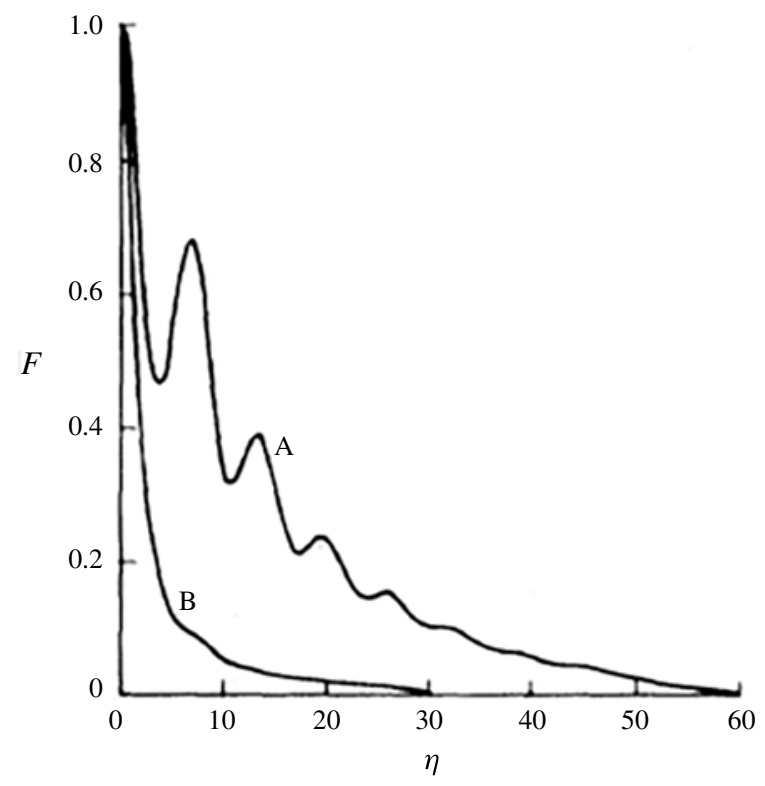

FIGURE 57. Marginally stable eigenfunctions for (A) low shear, $s=0.1$, and (B) high shear, $s=0.7$ (from Connor et al. 1978).

and $\alpha$ is a measure of the pressure gradient,

$$
\alpha=-\frac{2 R q^{2}}{B^{2}} \frac{\mathrm{d} p}{\mathrm{~d} r} .
$$

Note that $\omega^{2}$ has been set to zero and (28.1) is therefore the marginal stability equation. It is then clear from (28.3) that the ballooning threshold, or maximum stable $\beta$, will be of the form $\beta_{\max } \sim\left(a / R q^{2}\right) \alpha(s)$. We will see later (figure 58) that over a range of $s, \alpha(s) \sim 1 / 3$.

Numerical solutions of (28.1) for a low-shear and a high-shear equilibrium are shown in figure 57. In the low-shear case $F$ shows strong remnants of the periodic behaviour, but, as expected, these are suppressed in the high-shear case.

The stability boundary computed for the $s-\alpha$ model is shown in figure 58. In addition to the expected stability boundary '(a)', there is a second boundary '(b)'. Along the first boundary, $y_{0}=0$, corresponding to the usual mode 'ballooning' on the outer equator. However, along the second boundary, $y_{0}$ is not zero, nor is it the symmetric value $y_{0}=\pi$. In fact it is approximately $y_{0}=0.6 \pi$, corresponding to a mode 'ballooning' at an intermediate angle between the inner and outer equators. This is the result of the combined effect of normal and geodesic curvature. (The line '(c)' indicates where this boundary would be for $y_{0}=\pi$.)

Calculations for more realistic configurations include those for the JET experiment (Sykes et al. 1979). The result for an unstable JET plasma is shown in figure 59. Here the calculations are for the true, low-aspect-ratio, toroidal configuration, with the true D-shaped cross-section and realistic pressure profile. The figure includes the growth rates computed from the ballooning equation for the $n \rightarrow \infty$ limit, and the $1 / n$ corrections for large but finite $n$ (the spread in the $1 / n$ contribution reflects the limitations of numerical representation, and subsequent differentiations, 


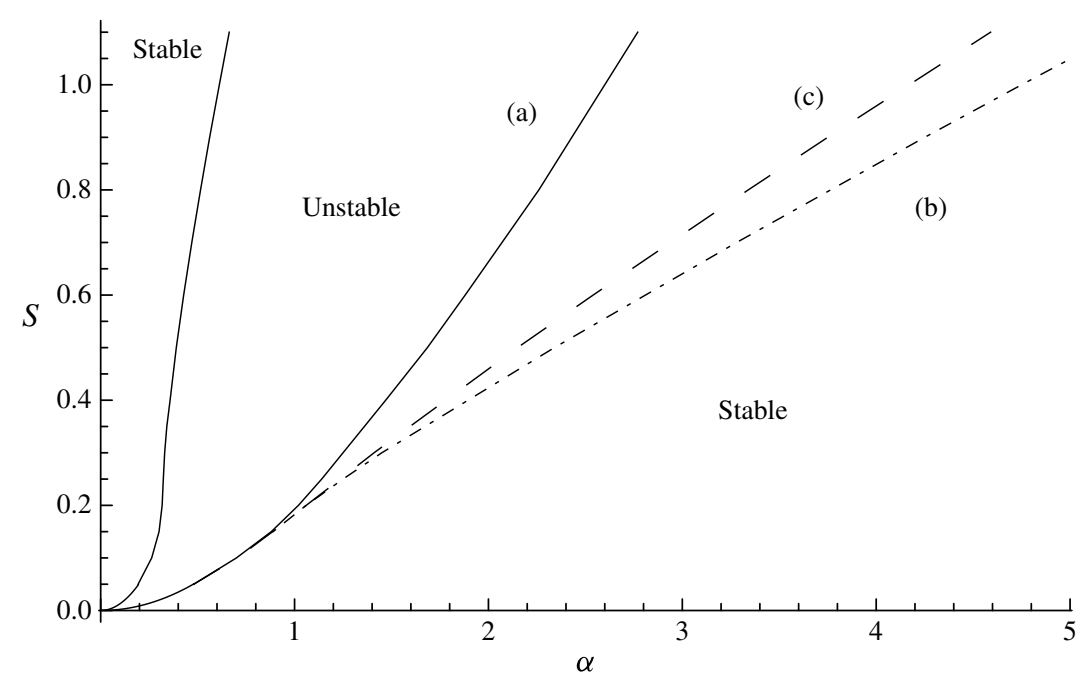

FIGURE 58. Stability boundaries for the $s-\alpha$ model obtained by taking (a) $y_{0}=0$ (solid line), (b) $y_{0}=0.6 \pi$ (dot-dashed line) and (c) $y_{0}=\pi$ (dashed line).

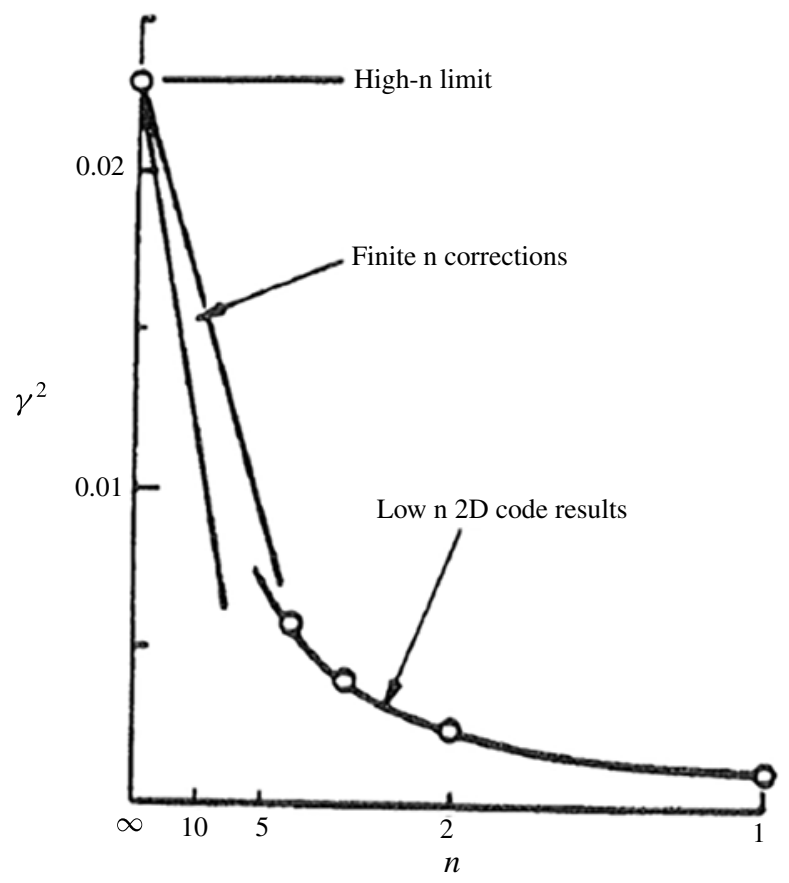

FIGURE 59. Variation of growth rate with toroidal mode number from direct MHD simulation for an unstable JET configuration, with central safety factor $q_{0}=0.75$, edge safety factor $q_{a}=6.4$ and average $\beta=0.045$. (Reproduced with permission from Sykes et al. (1979).) 


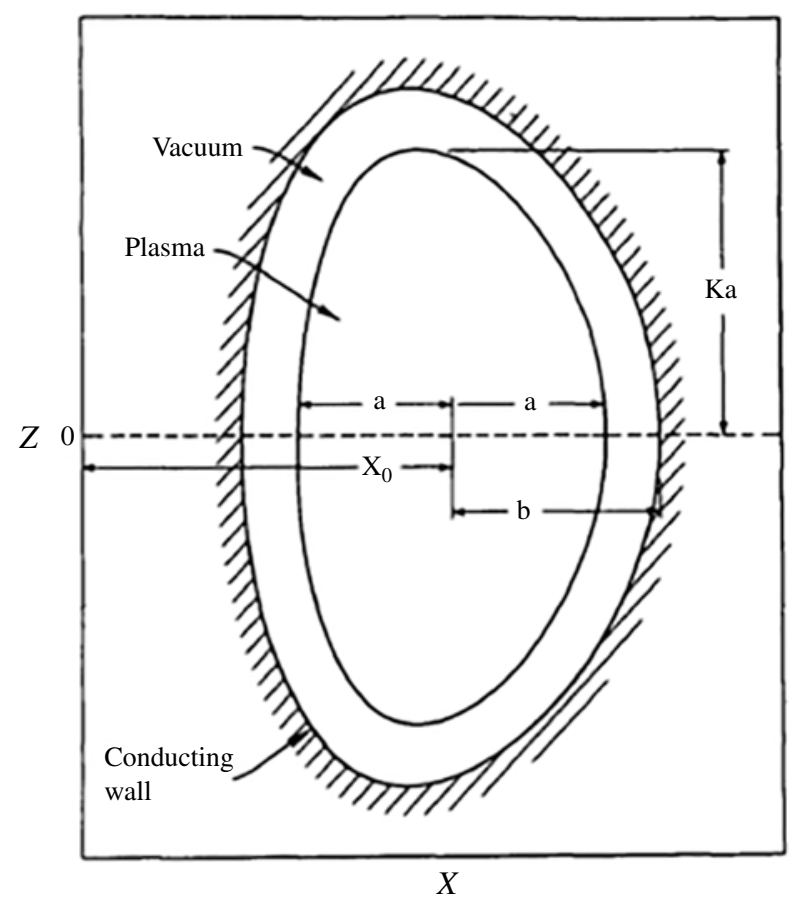

FIGURE 60. Geometry used to study ballooning stability. (Reproduced with permission from Todd et al. (1979).)

of the equilibrium profiles). The figure also shows the growth rate for low- $n$ modes $(n=1,2,3,4)$ computed using two-dimensional MHD codes. (These low- $n$ modes can be computed directly, as they do not require high resolution.) An important feature of figure 59 is that the growth rates $\gamma^{2}(n)$ calculated from the high- $n$ ballooning theory join smoothly to the low- $n$ computations, with the cross-over occurring at approximately $n=4$ or 5 . Thus we can be confident that our overall picture of ideal MHD toroidal modes is complete.

The effect of changing cross-section on ballooning modes was investigated by Todd et al. (1979) for plasma in a torus with the idealised D-shaped cross-section shown in figure 60 . The parameters $\delta$ and $\kappa$, respectively, define the triangularity and ellipticity of the boundary, $X=X_{0}+a \cos (\theta+\delta \sin \theta), Z=\kappa a \sin \theta$, and $\alpha$ describes the peaking of the pressure profile, $p(\Psi) \propto\left(\Psi_{b}-\Psi\right)^{\alpha}$. Figure 61 shows two examples of the maximum $\beta$ for modes of different $n$, both for plasma in a perfectly conducting shell, as in the previous examples, and for a 'free-boundary' plasma with the wall effectively at infinity. Here again the high- $n$ calculations link smoothly to the low- $n$ computations. Note that, in the free-boundary example in figure $61(b)$, the $n=1$ mode imposes a more stringent limit on $\beta$ than does the $n \rightarrow \infty$. But $n \rightarrow \infty$ is still the most stringent of the higher- $n$ modes as the theory requires.

\section{Validity of ballooning theory}

Now that we have seen the power and usefulness of the ballooning theory, it is appropriate to ask the following: When should perturbations in a torus be interpreted as ballooning modes and when should they be described by conventional Fourier 
(a)

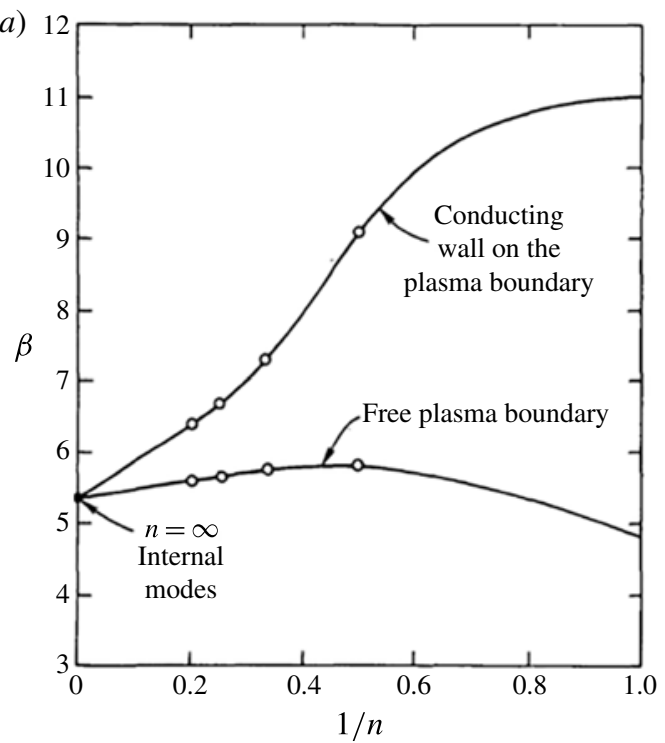

(b)

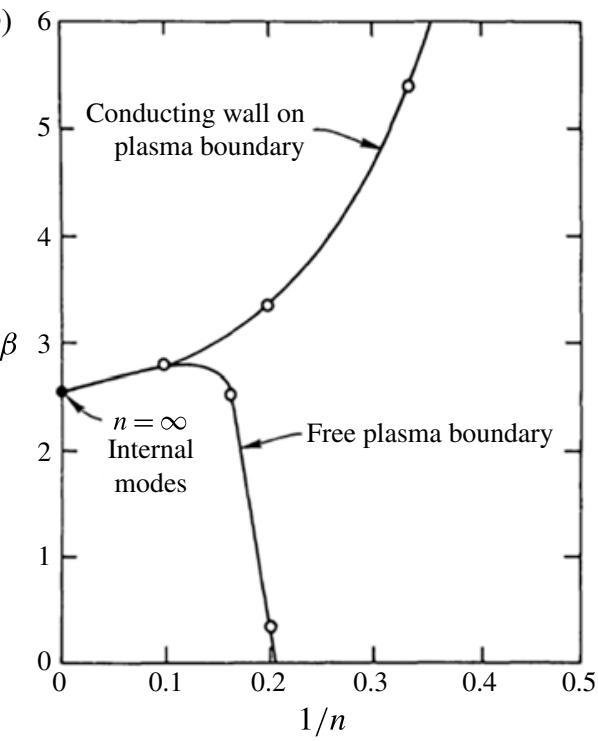

FIGURE 61. Critical $\beta$ as a function of $1 / n$ for a configuration with $(a) R / a=3.5, \kappa=$ 1.65, $\delta=0.25, \alpha=1.4$ and $1.0<q<3.0$ and $(b) R / a=4.6, \kappa=1.0, \delta=0, \alpha=2$ and $1.04<q<1.58$. (Reproduced with permission from Todd et al. (1979).)

modes - as in a straight cylinder (Connor \& Taylor 1987)? The parameter that determines this, which in the mathematical analysis we took to be ' $1 / n$ ', is physically

$$
g=\left(\frac{\text { coupling between Fourier modes resonant on adjacent surfaces }}{\text { difference in frequency between Fourier modes resonant on adjacent surfaces }}\right) \text {, }
$$

and ballooning theory is valid when $g$ is large.

The difference in frequency between Fourier modes is actually $\propto 1 / n s$, so that the theory is always valid as $n \rightarrow \infty$ unless the shear $s$ is small or vanishes (as it does in so-called 'reverse-shear' equilibria). A discussion of the 'small-shear' situation is given in Hastie \& Taylor (1981) and Connor \& Hastie (2004).

\section{Mode structure}

If we want to see the true structure of a ballooning mode, we must construct the physical perturbation $\phi$ from the 'fictitious' perturbation $\hat{\phi}$. That is, we must reverse the ballooning transformation (27.19) and (27.20) by evaluating the right-hand side of

$$
\phi(\Psi, \theta)=\sum_{m} \mathrm{e}^{-\mathrm{i} m \theta} \int_{-\infty}^{\infty} \mathrm{e}^{\mathrm{i} m y} \mathrm{e}^{-\mathrm{i} n q(\Psi) y} F(\Psi, y) \mathrm{d} y .
$$

Several interesting consequences follow from (30.1). If we carry out the integration over $y$, the result is clearly a function of $(m-n q)$ and

$$
\phi(\Psi, \theta)=\sum_{m} U(m-n q) \mathrm{e}^{-\mathrm{i} m \theta} .
$$




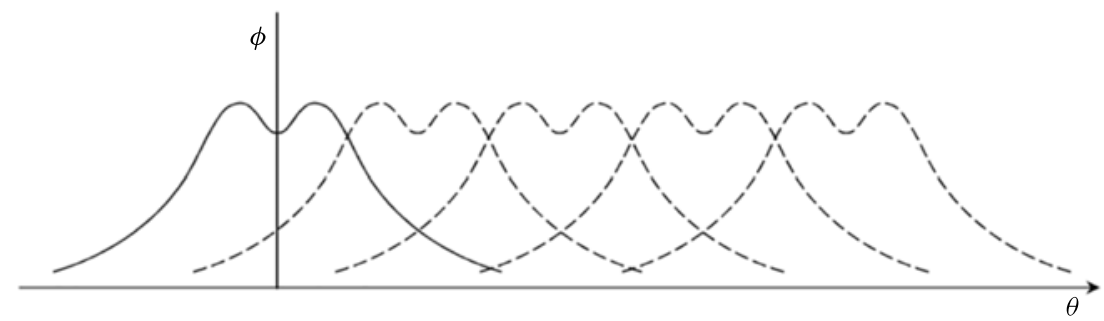

FIGURE 62. Constructing the ballooning perturbation from an infinite set of displaced functions.

This is a sum of similar contributions, each centred on a different rational surface, precisely as we would expect since neighbouring rational surfaces are almost degenerate after an appropriate realignment on each surface to compensate for the change in direction of $\boldsymbol{B}$; see $\S 25$. This degeneracy becomes exact as $n \rightarrow \infty$.

Equation (30.1) also provides another way of looking at the ballooning transform. If we write (30.1) as

$$
\phi(\Psi, \theta)=\int_{-\infty}^{\infty} \sum_{m} \mathrm{e}^{-\mathrm{i} m(\theta-y)} \mathrm{e}^{\mathrm{i} n q(\Psi) y} F(\Psi, y) \mathrm{d} y,
$$

and assume convergence, we can take the sum $\sum_{m} \mathrm{e}^{-\mathrm{i} m(\theta-y)}$ to be the Fourier series for the periodic function $\sum_{N} \delta(\theta-y-2 \pi N)$. Then

$$
\phi(\Psi, \theta)=\sum_{N} \mathrm{e}^{-\mathrm{in} q(\theta-2 \pi N)} F(\Psi, \theta-2 \pi N),
$$

in which the periodic perturbation is constructed from the non-periodic function $F$ by adding an infinite number of repetitions of it spaced at multiples of $2 \pi$ (see figure 62).

Yet another view is obtained by restoring the toroidal mode number $\exp (\mathrm{i} n \zeta)$, in each term of the sum (30.4). Each term then has the form

$$
F(\Psi, \theta) \mathrm{e}^{-\mathrm{i} n(q(\Psi) \theta-\zeta)},
$$

which is essentially the 'twisted slice' mentioned at the start of this study (see §25).

\section{Conclusion}

Before ballooning theory, only low- $n$ modes could be studied theoretically in a toroidal plasma, whereas in a cylindrical plasma all modes could be analysed from a single second-order ordinary differential equation. Ballooning theory makes it possible to study high- $n$ modes in a torus - again from a single second-order ordinary differential equation. In this sense the realistic toroidal problem has become as amenable to theoretical analysis as the idealised cylindrical one!

\section{Acknowledgements}

The contributions of R. J. Hastie to these lecture notes are gratefully acknowledged, as are numerous helpful discussions with J. W. Connor and C. J. Ham. This work has been part-funded by the RCUK Energy Programme (grant number EP/I501045). 


\section{REFERENCES}

Barnes, C. W., Fernández, J. C., Henins, I., Hoida, H. W., Jarboe, T. R., Knox, S. O., Marklin, G. J. \& MCKenna, K. F. 1986 Experimental determination of the conservation of magnetic helicity from the balance between source and spheromak. Phys. Fluids 29, $3415-3432$.

Bernstein, I. B., Frieman, E. A., Kruskal, M. D. \& Kulsrud, R. M. 1958 An energy principle for hydromagnetic stability problems. Proc. R. Soc. Lond. A 244, 17-40.

Bodin, H. A. B. 1983 Reversed field pinches. Nucl. Instrum. Methods 207, 1-22.

Bodin, H. A. B. 1984 Physics of reversed field pinch. In Proceedings of the International Conference on Plasma Physics, Lausanne (ed. M. Q. Tran \& R. J. Verbeek), vol. I, pp. 417-453. EEC.

Bodin, H. A. B. 1987 Results from the HBTX experiments. In Proceedings of the Course and Workshop on the Physics of Mirrors, Reversed Field Pinches and Compact Tori, Varenna (ed. S. Ortolani \& E. Sindoni), vol. I, pp. 307-329. International School of Plasma Physics.

Bodin, H. A. B.\& Newton, A. A. 1980 Reversed-field-pinch research. Nucl. Fusion 20, 1255-1324.

Brown, M. R. \& Bellan, P. M 1990 Spheromak injection into a tokamak. Phys. Fluids B 2, $1306-1310$.

Butt, E. P., Cole, H. C., Dellis, A. N. \& Wort, D. 1966 Conditions for improved stability in Zeta. In Proceedings of a Conference on Plasma Physics and Controlled Nuclear Fusion Research, Culham, 1965, vol. I, pp. 751-764. IAEA.

Catto, P. J., Tang, W. M. \& Baldwin, D. E. 1981 Generalized gyrokinetics. Plasma Phys. 23, 639-650.

Chan, V. S., Miller, R. L. \& OhKawa, T. 1990 Current drive by wave helicity injection. Phys. Fluids B 2, 1441-1445.

Connor, J. W., Hastie, R. J. \& TAYlor, J. B. 1978 Shear, periodicity and plasma ballooning modes. Phys. Rev. Lett. 40, 396-399.

Connor, J. W., HASTIE, R. J. \& TAYlor, J. B. 1979a High mode number stability of axisymmetric toroidal plasmas. In Proceedings of the 7th International Conference, Plasma Physics and Controlled Nuclear Fusion Research, Innsbruck, 1978, vol. I, pp. 667-674. IAEA.

Connor, J. W., Hastie, R. J. \& TAYlor, J. B. 1979b High mode number stability of an axisymmetric toroidal plasma. Proc. R. Soc. Lond. A 365, 1-17.

Connor, J. W. \& Hastie, R. J. 2004 The stability of ideal magnetohydrodynamic ballooning modes in plasma with internal transport barriers. Phys. Plasmas 11, 1520-1536.

CONNOR, J. W. \& TAYLOR, J. B. 1987 Ballooning modes of Fourier modes in a toroidal plasma? Phys. Fluids 30, 3180-3185.

Cooper, W. A. 1988 Ballooning instabilities in tokamaks with sheared toroidal flows. Plasma Phys. Control. Fusion 30, 1805-1812.

DAVIDson, R. C. 1972 Methods in Nonlinear Plasma Theory, p. 274. Academic.

DeWAR, R. L. \& Glasser, A. H. 1983 Ballooning mode spectrum in general toroidal systems. Phys. Fluids 26, 3038-3052.

DiMarco, J. N. 1983 The ZT-40M experiment. In Proceedings of the Course on Mirror-based and Field-reversed Approaches to Magnetic Fusion, Varenna, 1983 (ed. R. F. Post, D. E. Baldwin \& D. D. Ryutov), vol. II, pp. 681-703. International School of Plasma Physics.

Fernández, J. C., Wright, B. L., Marklin, G. J., Platts, D. A. \& Jarboe, T. R. 1989 The $m=1$ helicity source spheromak experiment. Phys. Fluids B 1, 1254-1270.

Freidberg, J. P. 1987 Ideal Magnetohydrodynamics, p. 251. Plenum.

Freidberg, J. P. 2014 Ideal MHD, p. 196. Cambridge University Press.

Furth, H. P., Killeen, J. \& Rosenbluth, M. N. 1963 Finite resistivity instabilities of a sheet pinch. Phys. Fluids 6, 459-484.

Gibson, A., Coxell, H., Powell, B. A. \& Reid, G. W. 1967 Plasma confinement during a period of reduced fluctuations in 'Zeta'. Plasma Phys. 9, 1-12.

Gimblett, C. G., Hall, P. J., Taylor, J. B. \& Turner, M. F. 1987 Eigenvalues associated with relaxed states of toroidal plasmas. Phys. Fluids 30, 3186-3195. 
Glasser, A. H. 1979 Ballooning modes in axisymmetric toroidal plasmas. In Proceedings of the Finite Beta Theory Workshop, Varenna, 1977 (ed. B. Coppi \& W. Sadowski), pp. 55-65. USDoE.

Goldenbaum, G. C., Irby, J. H., Chong, Y. P. \& Hart, G. W. 1980 Formation of a spheromak plasma configuration. Phys. Rev. Lett. 44, 393-396.

Goldstein, H. 1957 Classical Mechanics, p. 240. Addison-Wesley.

Gott, Yu. V., Ioffe, M. S. \& Tel'KovskiI, V. G. 1962 Some new results on confinement in a magnetic trap. Nucl. Fusion Suppl. Part 3, 1045-1047; (in Russian).

Grad, H. 1973 Magnetofluid-dynamic spectrum and low shear stability. Proc. Natl Acad. Sci. USA 70, 3277-3281.

GRAD, H. \& RUBIN, H. 1958 Hydromagnetic equilibria and force-free fields. In Proceedings of the Second United Nations International Conference on the Peaceful Uses of Atomic Energy, vol. 31, pp. 190-197. United Nations.

Greene, J. M. 1968 Two-dimensional measure-preserving mappings. J. Math. Phys. 9, 760-768.

Hart, G. W., Janos, A., Meyerhofer, D. D. \& Yamada, M. 1986 Verification of the Taylor (minimum energy) state in a spheromak. Phys. Fluids 29, 1994-1997.

Hastie, R. J., TAYlor, J. B. \& HaAs, F. A. 1967 Adiabatic invariants and the equilibrium of magnetically trapped particles. Ann. Phys. 41, 302-338.

Hastie, R. J., HobBs, G. D. \& TAYlor, J. B. 1969 Non-adiabatic behaviour of particles in inhomogeneous magnetic fields. In Proceedings of the 3rd International Conference, Plasma Physics and Controlled Nuclear Fusion Research, Novosibirsk, 1968, pp. 389-402. IAEA; paper $\mathrm{CN}-24 / \mathrm{C}-6$.

Hastie, R. J. 1993 Plasma particle dynamics. In Plasma Physics. An Introductory Course (ed. R. Dendy), p. 5. Cambridge University Press.

Hastie, R. J. \& TAYloR, J. B. 1981 Validity of ballooning representation and mode number dependence of stability. Nucl. Fusion 21, 187-191.

Hazeltine, R. D., Hitchcock, D. A. \& Mahajan, S. M. 1981 Uniqueness and inversion of the ballooning representation. Phys. Fluids 24, 180-181.

Hazeltine, R. D. \& Newcomb, W. A. 1990 Inversion of the ballooning transformation. Phys. Fluids B 2, 7-10.

HowARD, J. E. 1971 Nonadiabatic particle motion in cusped magnetic fields. Phys. Fluids 14, 2378-2384.

INCE, E. L. 1956 Ordinary Differential Equations, p. 223. Dover.

JANOS, A., HART, G. W. \& YAMADA, M. $1985 a$ Relaxation of spheromak plasmas toward a minimumenergy state and global magnetic fluctuations. Phys. Rev. Lett. 55, 2868-2871.

JAnOS, A., HART, G. W., NAM, C. H. \& YAMADA, M. 1985b Global magnetic fluctuations in spheromak plasmas and relaxation toward a minimum-energy state. Phys. Fluids 28, 3667-3675.

Jarboe, T. R., Barnes, C. W., Platts, D. A. \& Wright, B. L. 1985 A kinked Z-pinch as the helicity source for spheromak generation and sustainment. Comments Plasma Phys. Control. Fusion 9, 161-168.

Jensen, T. H. \& ChU, M. S. 1984 Current drive and helicity injection. Phys. Fluids 27, 2881-2885.

Johnson, J. L. et al. 1958 Some stable hydromagnetic equilibria. Phys. Fluids 1, 281-296.

Kitson, D. A. \& Browning, P. K. 1990 Partially relaxed magnetic field equilibria in a gun-injected spheromak. Plasma Phys. Control. Fusion 32, 1265-1287.

Kruskal, M. D. \& Oberman, C. R. 1958 On the stability of plasma in static equilibria. Phys. Fluids 1, 275-280.

La Haye, R. J., Jensen, T. H., Lee, P. S. C., Moore \& OhKawa, T. 1986 Multipinch - a reversed-field pinch with a magnetic well. Nucl. Fusion 26, 255-265.

LeE, Y. C. \& VAN DAM, J. W. 1979 Kinetic theory of ballooning instabilities. In Proceedings of the Finite Beta Theory Workshop, Varenna, 1977 (ed. B. Coppi \& W. Sadowski), pp. 93-101. USDoE.

Lichtenberg, A. \& Lieberman, M. 2010 Regular and Chaotic Dynamics, 2nd edn, p. 156, 218. Springer. 
Martin, T. J. \& TAYlor, J. B. 1974 Helically deformed states in toroidal pinches. Culham Laboratory Report (unpublished).

Mett, R. R. \& TAtaronis, J. A. 1989 Current drive by magnetohydrodynamic helicity waves. Phys. Rev. Lett. 63, 1380-1383.

Mett, R. R. \& TAYlor, J. B. 1992 Steady-state dynamo and current drive in a nonuniform bounded plasma. Phys. Plasmas 4, 73-78.

Moffat, H. K. 1978 Magnetic Field Generation in Electrically Conducting Fluids, p. 14. Cambridge University Press.

Newcomb, W. A. 1960 Hydromagnetic stability of a diffuse linear pinch. Ann. Phys. 10, 232-267.

Nishikawa, K. \& Wakatani, M. 1993 Plasma Physics, p. 40. Springer.

Post, R. F. 1983 The magnetic mirror principle as applied to fusion research. In Proceedings of the Course on Mirror-based and Field-reversed Approaches to Magnetic Fusion, Varenna, 1983 (ed. R. F. Post, D. E. Baldwin \& D. D. Ryutov), vol. II, pp. 7-41. International School of Plasma Physics.

Post, R. F. 1987 The magnetic mirror approach to fusion. Nucl. Fusion 27, 1579-1739.

Roberts, K. V.\& TAYLOR, J. B. 1965 Gravitational resistive instability of an incompressible plasma in a sheared magnetic field. Phys. Fluids 8, 315-322.

Rosenbluth, M. N. et al. 1966 Destruction of magnetic surfaces by magnetic field irregularities. Nucl. Fusion 6, 297-300.

Schoenberg, F., Ingraham, J. C., Munson, C. P., Weber, P. G., Baker, D. A., Gribble, R. F., Howell, R. B., Miller, G., Reass, W. A., Schofield, A. E., Shinohara, S. \& Wurden, G. A. 1988 Oscillating field current drive experiments in a reversed field pinch. Phys. Fluids 21, 2285-2291.

SPITZER, L. 1951 USAEC Reports; A proposed stellarator (NYO-993 PM-S-1); Particle orbits in a low-density stellarator (NYO-995 PM-S-3).

SPITZER, L. JR. 1958 The stellarator concept. In Proceedings of the Second United Nations International Conference on the Peaceful Uses of Atomic Energy, vol. 31, pp. 181-196. United Nations.

Sykes, A., Turner, M. F. F., Fielding, P. J. \& HAAs, F. A. 1979 MHD theory of ballooning modes applied to JET, and the effect of anisotropic pressure on ballooning modes. In Proceedings of the 7th International Conference, Plasma Physics and Controlled Nuclear Fusion Research, Innsbruck, 1978, vol. I, pp. 625-636. IAEA.

Tamaru, T., Sugisaki, K., Hayase, K., Shimada, T., Hirano, Y., Maejima, Y., Ogawa, K., Hirano, K., Kitagawa, S. \& SAto, K. 1979 Experimental and computational studies of high-beta tokamaks and reversed-field pinches. In Proceedings of the 7th International Conference, Plasma Physics and Controlled Nuclear Fusion Research, Innsbruck, 1978, vol. II, pp. 55-68. IAEA.

TAYlor, J. B. 1963 Some stable plasma equilibria in combined mirror-cusp fields. Phys. Fluids 6, $1529-1536$.

TAYLOR, J. B. 1964 Equilibrium and stability of plasma in arbitrary mirror fields. Phys. Fluids 7, $767-773$.

TAYlor, J. B. 1965 Plasma confinement in magnetic wells. In Plasma Physics: Lectures Presented at the 1964 Seminar on Plasma Physics Organised by and Held at the International Centre for Theoretical Physics, pp. 449-481. IAEA.

TAylor, J. B. 1968 Plasma containment and stability theory. Proc. R. Soc. Lond. A 304, 335-360.

TAYlOR, J. B. $1974 a$ Bundle divertors and topology. Culham Laboratory Report. CLM-R132. HMSO.

TAYLOR, J. B. 1974b Relaxation of toroidal plasma and generation of reverse magnetic fields. Phys. Rev. Lett. 33, 1139-1141.

TAYLOR, J. B. 1975 Relaxation of toroidal discharges to stable states and generation of reverse magnetic fields. In Proceedings of the 5th International Conference, Plasma Physics and Controlled Nuclear Fusion Research, Tokyo, 1974, vol. I, pp. 161-167. IAEA.

TAYlor, J. B. 1977 Does magnetic shear stabilize drift waves? In Proceedings of the 6th International Conference, Plasma Physics and Controlled Nuclear Fusion Research, Berchtesgarden, 1976, vol. II, pp. 323-329. IAEA. 
TAYLOR, J. B. 1985 Relaxation and magnetic reconnection in laboratory plasmas. Plasma Phys. Control. Fusion 27, 1439-1447.

TAYlor, J. B. 1986 Relaxation and magnetic reconnection in plasmas. Rev. Mod. Phys. 58, 741-763.

TAYLOR, J. B. 1989 Current drive by plasma waves and helicity conservation. Phys. Rev. Lett. 63, 1384-1385.

TAYLOR, J. B. 1992 Relaxation and topology in plasma experiments. In Topological Aspects of the Dynamics of Fluids and Plasmas, NATO ASI Series, pp. 151-165. Kluwer Academic.

TAYlor, J. B. 2000 Relaxation revisited. Phys. Plasmas 7, 1623-1629.

TAYlor, J. B. 2012 Ideal ballooning modes, shear flow and the stable continuum. Plasma Phys. Control. Fusion 54, 115005.

TAYlor, J. B. \& HAstie, R. J. 1965 Maximum plasma pressure for stability in magnetic fields with finite minima. Phys. Fluids 8, 323-332.

Todd, A. M. M., Manickam, J., Okabayashi, M., Chance, M. S., Grimm, R. C., Greene, J. M. \& Johnson, J. L. 1979 Dependence of ideal-MHD kink and ballooning modes in plasmas shape and profiles in tokamaks. Nucl. Fusion 19, 743-752.

Toyama, H., Asakura, N., Hattori, K., Inoue, N., Ishida, S., Matsuzuka, S., Miyamoto, K., Morikawa, J., Nagayama, Y., Nihei, H., Shinohara, S., Ueda, Y., Yamagishi, K. \& YoshidA, Z. 1985 Effect of bias toroidal field and filling pressure on the performance of the reversed field pinch device REPUTE-1. In Proceedings of the 12th European Conference on Controlled Fusion and Plasma Physics, Budapest (ed. L. Pocs \& A. Montvai), vol. I, pp. 602-605. European Physical Society.

Turner, W. C., Goldenbaum, G. C., Granneman, E. H. A., Hammer, J. H., Hartman, C. W., Prono, D. S. \& TASKA, J. 1983 Investigations of the magnetic structure and decay of a plasma-gun-generated compact torus. Phys. Fluids 26, 1965-1986.

TURNER, L. 1984 Analytic solutions of $\nabla \times \boldsymbol{B}=\lambda$ having separatrices for geometries with one ignorable coordinate. Phys. Fluids 27, 1677-1685.

WATt, R. G. \& Nebel, R. A. 1983 Sawteeth, magnetic disturbances, and magnetic flux regeneration in the reversed-field pinch. Phys. Fluids 26, 1168-1170.

Wesson, J. 2004 Tokamaks, 3rd edn p. 397. Oxford University Press.

Yamada, M., Furth, H. P., Hsu, W., Janos, A., Jardin, S., Okabayashu, M., Sinnis, J., StiX, T. H. \& YAMAZAKI, K. 1981 Quasistatic formation of the spheromak plasma configuration. Phys. Rev. Lett. 46, 188-191. 